\title{
A NEW CEMENT TO GLUE NON-CONFORMING GRIDS WITH ROBIN INTERFACE CONDITIONS: THE FINITE ELEMENT CASE
}

\author{
CAROLINE JAPHET*, YVON MADAY ${ }^{\dagger}$, AND FRÉDÉRIC NATAF
}

\begin{abstract}
We design and analyze a new non-conforming domain decomposition method based on Schwarz type approaches that allows for the use of Robin interface conditions on non-conforming grids. The method is proven to be well posed, and the iterative solver to converge. The error analysis is performed in $2 \mathrm{D}$ piecewise polynomials of low and high order and extended in $3 \mathrm{D}$ for $P_{1}$ elements. Numerical results in $2 \mathrm{D}$ illustrate the new method.
\end{abstract}

Key words. Optimized Schwarz domain decomposition, Robin transmission conditions, finite element methods, nonconforming grids, error analysis

1. Introduction. Our goal in writing this paper is to propose and analyze a non-conforming domain decomposition generalization to P.L. Lions initial idea 30 in view of an extension of the approach to optimized interface conditions algorithms. This type of algorithm has proven indeed to be an efficient approach to domain decomposition methods in the case of conforming approximations [14, 24. This paper presents the basic material related to so called zero ${ }^{\text {th }}$ order (non optimized) method in case of finite element discretizations, see 18 for a short presentation. In the companion paper 22, the case of the finite volume discretization has been introduced and analyzed.

We first consider the problem at the continuous level: Find $u$ such that

$$
\begin{gathered}
\mathcal{L}(u)=f \text { in } \Omega \\
\mathcal{C}(u)=g \text { on } \partial \Omega
\end{gathered}
$$

where $\mathcal{L}$ and $\mathcal{C}$ are partial differential equations. The original Schwarz algorithm is based on a decomposition of the domain $\Omega$ into overlapping subdomains and the resolution of Dirichlet boundary value problems in the subdomains. It has been proposed in [30. to use more general boundary conditions for the problems on the subdomains in order to use a non-overlapping decomposition of the domain. The convergence rate is also dramatically increased.

More precisely, let $\Omega$ be a $\mathcal{C}^{1,1}$ (or convex polygon in $2 \mathrm{D}$ or polyhedron in 3D) domain of $\mathbb{R}^{d}, d=2$ or 3 ; we assume it is decomposed into $K$ non-overlapping subdomains:

$$
\bar{\Omega}=\cup_{k=1}^{K} \bar{\Omega}^{k} .
$$

We suppose that the subdomains $\Omega^{k}, 1 \leq k \leq K$ are either $\mathcal{C}^{1,1}$ or convex polygons in $2 \mathrm{D}$ or polyhedrons in $3 \mathrm{D}$. We assume also that this decomposition is geometrically conforming in the sense that the intersection of the closure of two different subdomains,

\footnotetext{
*Laboratoire d'Analyse, Géométrie et Applications, Université Paris XIII, Avenue J-B Clément, 93430 Villetaneuse, France, E-mail: japhet@math.univ-paris13.fr

$\dagger$ Université Pierre et Marie Curie-Paris6, UMR 7598 Laboratoire Jacques-Louis Lions, B.C. 187, Paris, F-75005 France, E-mail: maday@ann.jussieu.fr; and Division of Applied Maths, Brown University

${ }^{\ddagger}$ Université Pierre et Marie Curie-Paris6, UMR 7598 Laboratoire Jacques-Louis Lions, B.C. 187, Paris, F-75005 France, E-mail: nataf@ann.jussieu.fr
} 
if not empty, is either a common vertex, a common edge, or a common face in 3D. Let $\mathbf{n}_{k}$ be the outward normal from $\Omega^{k}$. Let $\left(\mathcal{B}_{k, \ell}\right)_{1 \leq k, \ell \leq K, k \neq \ell}$ be the chosen transmission conditions on the interface between the subdomains (e.g. $\mathcal{B}_{k, \ell}=\frac{\partial}{\partial \mathbf{n}_{k}}+\alpha_{k}$ ). What we shall call here a Schwarz type method for the problem (1.1)-(1.2) is its reformulation: Find $\left(u_{k}\right)_{1 \leq k \leq K}$ such that

$$
\begin{array}{r}
\mathcal{L}\left(u_{k}\right)=f \text { in } \Omega^{k} \\
\mathcal{C}\left(u_{k}\right)=g \text { on } \partial \Omega^{k} \cap \partial \Omega \\
\mathcal{B}_{k, \ell}\left(u_{k}\right)=\mathcal{B}_{k, \ell}\left(u_{\ell}\right) \text { on } \partial \Omega^{k} \cap \partial \Omega^{\ell} .
\end{array}
$$

Let us focus first on the interface conditions $\mathcal{B}_{k, \ell}$. The convergence rate of associated Schwarz-type domain decomposition methods is very sensitive to the choice of these transmission conditions. The use of exact artificial (also called absorbing) boundary conditions as interface conditions leads to an optimal number of iterations, see [23, [32], 22] and 21]. Indeed, for a domain decomposed into $K$ strips, the number of iterations is $K$, see [32]. Let us remark that this result is rather surprising since exact absorbing conditions refer usually to truncation of infinite domains rather than interface conditions in domain decomposition. Nevertheless, this approach has some drawbacks:

1. the explicit form of these boundary conditions is known only for constant coefficient operators and simple geometries,

2. these boundary conditions are pseudo-differential. The cost per iteration is high since the corresponding discretization matrix is not sparse for the unknowns on the boundaries of the subdomains.

For this reason, it is usually preferred to use partial differential approximations to the exact absorbing boundary conditions. This approximation problem is classical in the field of computation on unbounded domains since the seminal paper of Engquist and Majda [17. The approximations correspond to "low frequency" approximations of the exact absorbing boundary conditions. In domain decomposition methods, many authors have used them for wave propagation problems [15, [16, [29, [6], [13, 27. and 9] and in fluid dynamics [31, [20. Instead of using "low frequency" in space approximations to the exact absorbing boundary conditions, it has been proposed to design approximations which minimize the convergence rate of the algorithm. Such optimization of the transmission conditions for the performance of the algorithm was done in, 24], 25] for a convection-diffusion equation, where coefficients in second order transmission conditions where optimized. These approximations are quite different from the "low frequency" approximations and increase dramatically the convergence rate of the method.

When the grids are conforming, the implementation of such interface conditions on the discretized problem is not too difficult. On the other hand, using non-conforming grids is very appealing since their use allows for parallel generation of meshes, for local adaptive meshes and fast and independent solvers. The mortar element method, first introduced in [8], enables the use of non-conforming grids. It is also well suited to the use of the so-called "Dirichlet-Neumann" (20]) or "Neumann-Neumann" preconditioned conjugate gradient method applied to the Schur complement matrix (28], [3], 35]). In the context of finite volume discretizations, it was proposed in 34 to use a mortar type method with arbitrary interface conditions. To our knowledge, such an approach has not been extended to a finite element discretization. Moreover, the approach we present here is different and simpler. 
Our final project is to use interface conditions such as OO2 interface conditions (see [24, 25]), this will be developed in a future paper. Here we consider only interface conditions of order $0: \mathcal{B}_{k, \ell}=\frac{\partial}{\partial \mathbf{n}_{k}}+\alpha_{k}$. The approach we propose and study was introduced in [18] and independently implemented in [26] for the Maxwell equations but without numerical analysis.

2. Definition of the method and of the iterative solver.. We consider the following problem : Find $u$ such that

$$
\begin{aligned}
(I d-\Delta) u=f & \text { in } \Omega \\
u=0 & \text { on } \partial \Omega
\end{aligned}
$$

where $f$ is given in $L^{2}(\Omega)$.

The variational statement of the problem (2.1)-(2.2) consists in writing the problem as follows : Find $u \in H_{0}^{1}(\Omega)$ such that

$$
\int_{\Omega}(\nabla u \nabla v+u v) d x=\int_{\Omega} f v d x, \quad \forall v \in H_{0}^{1}(\Omega) .
$$

Making use of the domain decomposition (1.3), the problem (2.3) can be written as follows : Find $u \in H_{0}^{1}(\Omega)$ such that

$$
\sum_{k=1}^{K} \int_{\Omega^{k}}\left(\nabla\left(u_{\mid \Omega^{k}}\right) \nabla\left(v_{\mid \Omega^{k}}\right)+u_{\mid \Omega^{k}} v_{\mid \Omega^{k}}\right) d x=\sum_{k=1}^{K} \int_{\Omega^{k}} f_{\mid \Omega^{k}} v_{\mid \Omega^{k}} d x, \quad \forall v \in H_{0}^{1}(\Omega) .
$$

Let us introduce the space $H_{*}^{1}\left(\Omega^{k}\right)$ defined by

$$
H_{*}^{1}\left(\Omega^{k}\right)=\left\{\varphi \in H^{1}\left(\Omega^{k}\right), \quad \varphi=0 \text { over } \partial \Omega \cap \partial \Omega^{k}\right\} .
$$

It is standard to note that the space $H_{0}^{1}(\Omega)$ can then be identified with the subspace of the $K$-tuple $\underline{v}=\left(v_{1}, \ldots, v_{K}\right)$ that are continuous on the interfaces:

$$
\begin{array}{r}
V=\left\{\underline{v}=\left(v_{1}, \ldots, v_{K}\right) \in \prod_{k=1}^{K} H_{*}^{1}\left(\Omega^{k}\right),\right. \\
\left.\forall k, \ell, k \neq \ell, 1 \leq k, \ell \leq K, v_{k}=v_{\ell} \text { over } \partial \Omega^{k} \cap \partial \Omega^{\ell}\right\} .
\end{array}
$$

This leads to introduce also the notation of the interfaces of two adjacent subdomains

$$
\Gamma^{k, \ell}=\partial \Omega^{k} \cap \partial \Omega^{\ell} .
$$

In what follows, for the sake of simplicity, the only fact to refer to a pair $(k, \ell)$ preassumes that $\Gamma^{k, \ell}$ is not empty. The problem (2.3) is then equivalent to the following one : Find $\underline{u} \in V$ such that

$$
\sum_{k=1}^{K} \int_{\Omega^{k}}\left(\nabla u_{k} \nabla v_{k}+u_{k} v_{k}\right) d x=\sum_{k=1}^{K} \int_{\Omega^{k}} f_{k} v_{k} d x, \quad \forall \underline{v} \in V .
$$

Lemma 1. For $\underline{v} \in \prod_{k=1}^{K} H_{*}^{1}\left(\Omega^{k}\right)$, the constraint $v_{k}=v_{\ell}$ across the interface $\Gamma^{k, \ell}$ is equivalent to

$$
\begin{aligned}
& \forall \underline{p} \equiv\left(p_{k}\right) \in \prod_{k=1}^{K} H^{-1 / 2}\left(\partial \Omega^{k}\right) \text { with } p_{k}=-p_{\ell} \text { over } \Gamma^{k, \ell}, \\
& \qquad \sum_{k=1}^{K} H^{-1 / 2}\left(\partial \Omega^{k}\right)<p_{k}, v_{k}>_{H^{1 / 2}\left(\partial \Omega^{k}\right)}=0 .
\end{aligned}
$$


Proof The proof is similar to the one of proposition III.1.1 in [12 but can't be directly derived from this proposition. Let $\underline{p} \equiv\left(p_{k}\right) \in \prod_{k=1}^{K} H^{-1 / 2}\left(\partial \Omega^{k}\right)$ with $p_{k}=$ $-p_{\ell}$ over $\Gamma^{k, \ell}$, in $\left(H_{00}^{1 / 2}\left(\Gamma^{k, \ell}\right)\right)^{\prime}$ sense. Then, there exists over each $\Omega^{k}$ a lifting of the normal trace $p_{k}$ in $H\left(\operatorname{div}, \Omega^{k}\right)$. The global function $\mathbf{P}$, which restriction to each $\Omega^{k}$ is defined as being equal to the lifting, belongs to $H(\operatorname{div}, \Omega)$ and is such that $(\mathbf{P . n})_{\mid \partial \Omega^{k}}=p_{k}$. Let now $\underline{v} \in V$. From the previously quoted identification, we know that there exists $\mathbf{v} \in H_{0}^{1}(\Omega)$ such that $\mathbf{v}_{\mid \Omega^{k}}=v_{k}$. In addition,

$$
\int_{\Omega} \mathbf{v} \operatorname{div} \mathbf{P}-\int_{\Omega} \mathbf{P} \nabla \mathbf{v}=0
$$

On the other hand,

$$
\begin{aligned}
\int_{\Omega} \mathbf{v} \operatorname{div} \mathbf{P}-\int_{\Omega} \mathbf{P} \nabla \mathbf{v} & =\sum_{k=1}^{K}\left(\int_{\Omega^{k}} \mathbf{v} \operatorname{div} \mathbf{P}-\int_{\Omega^{k}} \mathbf{P} \nabla \mathbf{v}\right) \\
& =\sum_{k=1}^{K} \int_{\partial \Omega^{k}}(\mathbf{P} . \mathbf{n}) \underline{v}=\sum_{k=1}^{K} \int_{\partial \Omega^{k}} p_{k} v_{k}
\end{aligned}
$$

so that (2.4) is satisfied.

Reciprocally, let $\underline{v}=\left(v_{1}, \ldots, v_{K}\right) \in \prod_{k=1}^{K} H_{*}^{1}\left(\Omega^{k}\right)$ such that (2.4) is satisfied. Let $x \in \Gamma^{k, \ell}$, and let $\gamma_{x} \subset \bar{\gamma}_{x} \subset \Gamma_{x} \subset \bar{\Gamma}_{x} \subset \Gamma^{k, \ell}$ be open sets. There exists a function $\varphi$ in $\mathcal{D}\left(\Gamma_{x}\right)$ such that $\varphi(y)=1$ for all $y$ in $\gamma_{x}$. With any $q \in\left(H_{00}^{1 / 2}\left(\Gamma_{x}\right)\right)^{\prime}$, let us associate $\underline{p} \equiv\left(p_{k}\right)$ defined by

$$
\begin{aligned}
& H^{-1 / 2}\left(\partial \Omega^{k}\right)<p_{k}, w_{k}>_{H^{1 / 2}\left(\partial \Omega^{k}\right)}={ }_{\left(H_{00}^{1 / 2}\left(\Gamma_{x}\right)\right)^{\prime}}<q, \varphi w_{k}>_{H_{00}^{1 / 2}\left(\Gamma_{x}\right)}, \forall w_{k} \in H^{1 / 2}\left(\partial \Omega^{k}\right), \\
& H^{-1 / 2}\left(\partial \Omega^{\ell}\right)<p_{\ell}, w_{\ell}>_{H^{1 / 2}\left(\partial \Omega^{\ell}\right)}=-_{\left(H_{00}^{1 / 2}\left(\Gamma_{x}\right)\right)^{\prime}}<q, \varphi w_{\ell}>_{H_{00}^{1 / 2}\left(\Gamma_{x}\right)}, \forall w_{\ell} \in H^{1 / 2}\left(\partial \Omega^{\ell}\right), \\
& \text { and } p_{j}=0, \forall j \neq k, \ell \text {. }
\end{aligned}
$$

Then, by construction, $\underline{p} \in \prod_{k=1}^{K} H^{-1 / 2}\left(\partial \Omega^{k}\right)$ and $p_{k}=-p_{\ell}$ over $\Gamma^{k, \ell}$. Hence from (2.4),

$$
\sum_{k=1}^{K} H^{-1 / 2}\left(\partial \Omega^{k}\right)<p_{k}, v_{k}>_{H^{1 / 2}\left(\partial \Omega^{k}\right)}=0 .
$$

We derive

$$
H^{-1 / 2}\left(\partial \Omega^{k}\right)<p_{k}, v_{k}>_{H^{1 / 2}\left(\partial \Omega^{k}\right)}=-H_{H^{-1 / 2}\left(\partial \Omega^{\ell}\right)}<p_{\ell}, v_{\ell}>_{H^{1 / 2}\left(\partial \Omega^{\ell}\right)},
$$

thus,

$$
\left(H_{00}^{1 / 2}\left(\Gamma_{x}\right)\right)^{\prime}<q, \varphi v_{k}>_{H_{00}^{1 / 2}\left(\Gamma_{x}\right)}={ }_{\left(H_{00}^{1 / 2}\left(\Gamma_{x}\right)\right)^{\prime}}<q, \varphi v_{\ell}>_{H_{00}^{1 / 2}\left(\Gamma_{x}\right)},
$$

and this is true for any $q \in\left(H_{00}^{1 / 2}\left(\Gamma_{x}\right)\right)^{\prime}$, hence $\varphi v_{k}=\varphi v_{\ell}$ over $\Gamma_{x}$, and thus

$$
v_{k}=v_{\ell} \text { over } \gamma_{x}, \quad \forall x \in \Gamma^{k, \ell} .
$$

We derive $v_{k}=v_{\ell}$ a.e. over $\Gamma^{k, \ell}$, which ends the proof of lemma 1 . 
The constrained space is then defined as follows

$$
\begin{array}{r}
\mathcal{V}=\left\{(\underline{v}, \underline{q}) \in\left(\prod_{k=1}^{K} H_{*}^{1}\left(\Omega^{k}\right)\right) \times\left(\prod_{k=1}^{K} H^{-1 / 2}\left(\partial \Omega^{k}\right)\right),\right. \\
\left.v_{k}=v_{\ell} \text { and } q_{k}=-q_{\ell} \text { over } \Gamma^{k, \ell}\right\}
\end{array}
$$

and problem (2.3) is equivalent to the following one: Find $(\underline{u}, \underline{p}) \in \mathcal{V}$ such that

$\forall \underline{v} \in \prod_{k=1}^{K} H_{*}^{1}\left(\Omega^{k}\right)$,

$$
\begin{aligned}
\sum_{k=1}^{K} \int_{\Omega^{k}}\left(\nabla u_{k} \nabla v_{k}+u_{k} v_{k}\right) d x-\sum_{k=1}^{K} H^{-1 / 2}\left(\partial \Omega^{k}\right) & <p_{k}, v_{k}>_{H^{1 / 2}\left(\partial \Omega^{k}\right)} \\
& =\sum_{k=1}^{K} \int_{\Omega^{k}} f_{k} v_{k} d x .
\end{aligned}
$$

Being equivalent with the original problem, where $p_{k}=\frac{\partial u}{\partial \mathbf{n}_{k}}$ over $\partial \Omega^{k}$ (remind that $f$ is assumed to be in $L^{2}(\Omega)$ so that $\frac{\partial u}{\partial \mathbf{n}_{k}}$ actually belongs to $H^{-1 / 2}\left(\partial \Omega^{k}\right)$ ), this problem is naturally well posed. This can also be directly derived from the proof of an inf-sup condition that follows from the arguments developed hereafter for the analysis of the iterative procedure. First, let us describe this algorithm in the continuous case, and then in the non conforming discrete case. In both cases, we prove the convergence of the algorithm towards the solution of the problem.

2.1. Continuous case. Let us consider the interface conditions of order 0 :

$$
p_{k}+\alpha u_{k}=-p_{\ell}+\alpha u_{\ell} \quad \text { over } \Gamma^{k, \ell}
$$

where $\alpha$ is a given positive real number.

We introduce the following algorithm : let $\left(u_{k}^{n}, p_{k}^{n}\right) \in H_{*}^{1}\left(\Omega^{k}\right) \times H^{-1 / 2}\left(\partial \Omega^{k}\right)$ be an approximation of $(u, p)$ in $\Omega^{k}$ at step $n$. Then, $\left(u_{k}^{n+1}, p_{k}^{n+1}\right)$ is the solution in $H_{*}^{1}\left(\Omega^{k}\right) \times H^{-1 / 2}\left(\partial \Omega^{k}\right)$ of

$$
\begin{array}{r}
\int_{\Omega^{k}}\left(\nabla u_{k}^{n+1} \nabla v_{k}+u_{k}^{n+1} v_{k}\right) d x-{ }_{H^{-1 / 2}\left(\partial \Omega^{k}\right)}<p_{k}^{n+1}, v_{k}>_{H^{1 / 2}\left(\partial \Omega^{k}\right)} \\
=\int_{\Omega^{k}} f_{k} v_{k} d x, \quad \forall v_{k} \in H_{*}^{1}\left(\Omega^{k}\right) \\
<p_{k}^{n+1}+\alpha u_{k}^{n+1}, v_{k}>_{\Gamma^{k, \ell}}=<-p_{\ell}^{n}+\alpha u_{\ell}^{n}, v_{k}>_{\Gamma^{k, \ell}}, \quad \forall v_{k} \in H_{00}^{1 / 2}\left(\Gamma^{k, \ell}\right)
\end{array}
$$

It is obvious to remark that this series of equations results in uncoupled problems set on every $\Omega^{k}$. Recalling that $f \in L^{2}(\Omega)$, the strong formulation is indeed that

$$
\begin{array}{rr}
-\Delta u_{k}^{n+1}+u_{k}^{n+1}=f_{k} & \text { over } \Omega^{k} \\
\frac{\partial u_{k}^{n+1}}{\partial \mathbf{n}_{k}}+\alpha u_{k}^{n+1}=-p_{\ell}^{n}+\alpha u_{\ell}^{n} & \text { over } \Gamma^{k, \ell} \\
p_{k}^{n+1}=\frac{\partial u_{k}^{n+1}}{\partial \mathbf{n}_{k}} & \text { over } \partial \Omega^{k}
\end{array}
$$

From this strong formulation it is straightforward to derive by induction that if each $p_{k}^{0}, k=1, \ldots, K$, is chosen in $\prod_{\ell} H^{1 / 2}\left(\Gamma^{k, \ell}\right)$, then, for each $k, 1 \leq k \leq K$, and $n \geq 0$ 
the solution $u_{k}^{n+1}$ belongs to $H^{1}\left(\Omega^{k}\right)$ and $p_{k}^{n+1}$ belongs to $\prod_{\ell} H^{1 / 2}\left(\Gamma^{k, \ell}\right)$ by standard trace results $\left(p_{k}^{n+1}=-p_{\ell}^{n}+\alpha\left(u_{\ell}^{n}-u_{k}^{n+1}\right)\right)$. This regularity assumption on $p_{k}^{0}$ will be done hereafter.

We can prove now that the algorithm (2.5)-(2.6) converges for all $f \in L^{2}(\Omega)$. As the equations are linear, we can take $f=0$. We prove the convergence in the sense that, in this case, the associated sequence $\left(\left(u_{k}^{n}, p_{k}^{n}\right)\right)_{n}$ satisfies

$$
\lim _{n \longrightarrow \infty}\left(\left\|u_{k}^{n}\right\|_{H^{1}\left(\Omega^{k}\right)}+\left\|p_{k}^{n}\right\|_{H^{-1 / 2}\left(\partial \Omega^{k}\right)}\right)=0, \text { for } 1 \leq k \leq K .
$$

We proceed as in $([30,[14])$ by using an energy estimate that we derive by taking $v_{k}=u_{k}^{n+1}$ in (2.5) and the use of the regularity property that $p_{k}^{n+1} \in L^{2}\left(\partial \Omega^{k}\right)$

$$
\int_{\Omega^{k}}\left(\left|\nabla u_{k}^{n+1}\right|^{2}+\left|u_{k}^{n+1}\right|^{2}\right) d x=\int_{\partial \Omega^{k}} p_{k}^{n+1} u_{k}^{n+1} d s
$$

that can also be written

$$
\begin{aligned}
\int_{\Omega^{k}}\left(\left|\nabla u_{k}^{n+1}\right|^{2}+\left|u_{k}^{n+1}\right|^{2}\right) d x & \\
& =\sum_{\ell} \frac{1}{4 \alpha} \int_{\Gamma^{k, \ell}}\left(\left(p_{k}^{n+1}+\alpha u_{k}^{n+1}\right)^{2}-\left(p_{k}^{n+1}-\alpha u_{k}^{n+1}\right)^{2}\right) d s
\end{aligned}
$$

By using the interface conditions (2.6) we obtain

$$
\begin{aligned}
\int_{\Omega^{k}}\left(\left|\nabla u_{k}^{n+1}\right|^{2}+\left|u_{k}^{n+1}\right|^{2}\right) d x+ & \frac{1}{4 \alpha} \sum_{\ell} \int_{\Gamma^{k, \ell}}\left(p_{k}^{n+1}-\alpha u_{k}^{n+1}\right)^{2} d s \\
& =\frac{1}{4 \alpha} \sum_{\ell} \int_{\Gamma^{k, \ell}}\left(-p_{\ell}^{n}+\alpha u_{\ell}^{n}\right)^{2} d s
\end{aligned}
$$

Let us now introduce two new quantities defined at each step $n$ :

$$
E^{n}=\sum_{k=1}^{K} \int_{\Omega^{k}}\left(\left|\nabla u_{k}^{n}\right|^{2}+\left|u_{k}^{n}\right|^{2}\right),
$$

and

$$
B^{n}=\frac{1}{4 \alpha} \sum_{k=1}^{K} \sum_{\ell \neq k} \int_{\Gamma^{k, \ell}}\left(p_{k}^{n}-\alpha u_{k}^{n}\right)^{2} d s .
$$

By summing up the estimates (2.8) over $k=1, \ldots, K$, we have,

$$
E^{n+1}+B^{n+1} \leq B^{n}
$$

so that, by summing up these inequalities, now over $n$, we obtain :

$$
\sum_{n=1}^{\infty} E^{n} \leq B^{0}
$$

We thus have $\lim _{n \longrightarrow \infty} E^{n}=0$. Relation (2.7) then implies :

$$
\lim _{n \longrightarrow \infty}\left\|p_{k}^{n}\right\|_{H^{-1 / 2}\left(\partial \Omega^{k}\right)}=0, \text { for } k=1, \ldots, K .
$$


which ends the proof of the convergence of the continuous algorithm.

TheOREm 1. Assume that $f$ is in $L^{2}(\Omega)$ and $\left(p_{k}^{0}\right)_{1 \leq k \leq K} \in \prod_{\ell} H^{1 / 2}\left(\Gamma^{k, \ell}\right)$. Then, the algorithm (2.5)-(2.6) converges in the sense that

$$
\lim _{n \longrightarrow \infty}\left(\left\|u_{k}^{n}-u_{k}\right\|_{H^{1}\left(\Omega^{k}\right)}+\left\|p_{k}^{n}-p_{k}\right\|_{H^{-1 / 2}\left(\partial \Omega^{k}\right)}\right)=0, \text { for } 1 \leq k \leq K,
$$

where $u_{k}$ is the restriction to $\Omega^{k}$ of the solution $u$ to (2.1)-(2.2), and $p_{k}=\frac{\partial u_{k}}{\partial \mathbf{n}_{k}}$ over $\partial \Omega^{k}, 1 \leq k \leq K$.

2.2. Discrete case. We introduce now the discrete spaces. Each $\Omega^{k}$ is provided with its own mesh $\mathcal{T}_{h}^{k}, 1 \leq k \leq K$, such that

$$
\bar{\Omega}^{k}=\cup_{T \in \mathcal{T}_{h}^{k}} T .
$$

For $T \in \mathcal{T}_{h}^{k}$, let $h_{T}$ be the diameter of $T\left(h_{T}=\sup _{x, y \in T} d(x, y)\right)$ and $h$ the discretization parameter

$$
h=\max _{1 \leq k \leq K} h_{k}, \quad \text { with } \quad h_{k}=\max _{T \in \mathcal{T}_{h}^{k}} h_{T} .
$$

At the price of (even) more techniques and care in the forthcomming analysis, possible large variations in the norms of the solution $u_{\mid \Omega^{k}}$ can be compensated by tuning of $h_{k}$. This requires that the uniform $h$ is not used but all the analysis is performed with $h_{k}$. For the sake of readability we prefer to use $h$ instead of $h_{k}$. Let $\rho_{T}$ be the diameter of the circle (in 2D) or sphere (in 3D) inscribed in $T$, then $\sigma_{T}=\frac{h_{T}}{\rho_{T}}$ is a measure of the nondegeneracy of $T$. We suppose that $\mathcal{T}_{h}^{k}$ is uniformly regular: there exists $\sigma$ and $\tau$ independent of $h$ such that

$$
\forall T \in \mathcal{T}_{h}^{k}, \quad \sigma_{T} \leq \sigma \quad \text { and } \quad \tau h \leq h_{T} .
$$

We consider that the sets belonging to the meshes are of simplicial type (triangles or tetrahedron), but the analysis made hereafter can be applied as well for quadrangular or hexahedral meshes. Let $\mathcal{P}_{M}(T)$ denote the space of all polynomials defined over $\mathrm{T}$ of total degree less than or equal to $M$. The finite elements are of lagrangian type, of class $\mathcal{C}^{0}$. Then, we define over each subdomain two conforming spaces $Y_{h}^{k}$ and $X_{h}^{k}$ by :

$$
\begin{aligned}
& Y_{h}^{k}=\left\{v_{h, k} \in \mathcal{C}^{0}\left(\bar{\Omega}^{k}\right), \quad v_{h, k \mid T} \in \mathcal{P}_{M}(T), \forall T \in \mathcal{T}_{h}^{k}\right\},
\end{aligned}
$$

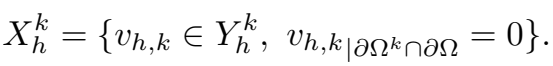

In what follows we assume that the mesh is designed by taking into account the geometry of the $\Gamma^{k, \ell}$ in the sense that, the space of traces over each $\Gamma^{k, \ell}$ of elements of $Y_{h}^{k}$ is a finite element space denoted by $\mathcal{Y}_{h}^{k, \ell}$. Again, at the price of more notations, this assumption can be relaxed. Let $k$ be given, the space $\mathcal{Y}_{h}^{k}$ is then the product space of the $\mathcal{Y}_{h}^{k, \ell}$ over each $\ell$ such that $\Gamma^{k, \ell} \neq \emptyset$. With each such interface we associate a subspace $\tilde{W}_{h}^{k, \ell}$ of $\mathcal{Y}_{h}^{k, \ell}$ in the same spirit as in the mortar element method [8] in $2 \mathrm{D}$ or [5] and [11] in 3D. To be more specific, let us recall the situation in 2D. If the space $X_{h}^{k}$ consist of continuous piecewise polynomials of degree $\leq M$, then it is readily noticed that the restriction of $X_{h}^{k}$ to $\Gamma^{k, \ell}$ consists in finite element functions adapted to the (possibly curved) side $\Gamma^{k, \ell}$ of piecewise polynomials of degree $\leq M$. This side 
has two end points that we denote as $x_{0}^{k, \ell}$ and $x_{n}^{k, \ell}$ that belong to the set of vertices of the corresponding triangulation of $\Gamma^{k, \ell}: x_{0}^{k, \ell}, x_{1}^{k, \ell}, \ldots, x_{n-1}^{k, \ell}, x_{n}^{k, \ell}$. The space $\tilde{W}_{h}^{k, \ell}$ is then the subspace of those elements of $\mathcal{Y}_{h}^{k, \ell}$ that are polynomials of degree $\leq M-1$ over both $\left[x_{0}^{k, \ell}, x_{1}^{k, \ell}\right]$ and $\left[x_{n-1}^{k, \ell}, x_{n}^{k, \ell}\right]$. As before, the space $\tilde{W}_{h}^{k}$ is the product space of the $\tilde{W}_{h}^{k, \ell}$ over each $\ell$ such that $\Gamma^{k, \ell} \neq \emptyset$.

The discrete constrained space is then defined as

$$
\begin{array}{r}
\mathcal{V}_{h}=\left\{\left(\underline{u}_{h}, \underline{p}_{h}\right) \in\left(\prod_{k=1}^{K} X_{h}^{k}\right) \times\left(\prod_{k=1}^{K} \tilde{W}_{h}^{k}\right),\right. \\
\left.\int_{\Gamma^{k, \ell}}\left(\left(p_{h, k}+\alpha u_{h, k}\right)-\left(-p_{h, \ell}+\alpha u_{h, \ell}\right)\right) \psi_{h, k, \ell}=0, \forall \psi_{h, k, \ell} \in \tilde{W}_{h}^{k, \ell}\right\},
\end{array}
$$

and the discrete problem is the following one : Find $\left(\underline{u}_{h}, \underline{p}_{h}\right) \in \mathcal{V}_{h}$ such that

$$
\begin{aligned}
& \forall \underline{v}_{h}=\left(v_{h, 1}, \ldots v_{h, K}\right) \in \prod_{k=1}^{K} X_{h}^{k}, \\
& \sum_{k=1}^{K} \int_{\Omega^{k}}\left(\nabla u_{h, k} \nabla v_{h, k}+u_{h, k} v_{h, k}\right) d x-\sum_{k=1}^{K} \int_{\partial \Omega^{k}} p_{h, k} v_{h, k} d s=\sum_{k=1}^{K} \int_{\Omega^{k}} f_{k} v_{h, k} d x .
\end{aligned}
$$

We introduce the discrete algorithm : let $\left(u_{h, k}^{n}, p_{h, k}^{n}\right) \in X_{h}^{k} \times \tilde{W}_{h}^{k}$ be a discrete approximation of $(u, p)$ in $\Omega^{k}$ at step $n$. Then, $\left(u_{h, k}^{n+1}, p_{h, k}^{n+1}\right)$ is the solution in $X_{h}^{k} \times \tilde{W}_{h}^{k}$ of

$$
\begin{array}{r}
\int_{\Omega^{k}}\left(\nabla u_{h, k}^{n+1} \nabla v_{h, k}+u_{h, k}^{n+1} v_{h, k}\right) d x-\int_{\partial \Omega^{k}} p_{h, k}^{n+1} v_{h, k} d s=\int_{\Omega^{k}} f_{k} v_{h, k} d x, \forall v_{h, k} \in X_{h}^{k} \\
\int_{\Gamma^{k, \ell}}\left(p_{h, k}^{n+1}+\alpha u_{h, k}^{n+1}\right) \psi_{h, k, \ell}=\int_{\Gamma^{k, \ell}}\left(-p_{h, \ell}^{n}+\alpha u_{h, \ell}^{n}\right) \psi_{h, k, \ell}, \quad \forall \psi_{h, k, \ell} \in \tilde{W}_{h}^{k, \ell} .
\end{array}
$$

In order to analyze the convergence of this iterative scheme, we have to precise the norms that can be used on the Lagrange multipliers $\underline{p}_{h}$. For any $\underline{p} \in \prod_{k=1}^{K} L^{2}\left(\partial \Omega^{k}\right)$, in addition to the natural norm, we can define two better suited norms as follows

$$
\|\underline{p}\|_{-\frac{1}{2}, *}=\left(\sum_{k=1}^{K} \sum_{\substack{\ell=1 \\ \ell \neq k}}^{K}\left\|p_{k}\right\|_{H_{*}^{-\frac{1}{2}}\left(\Gamma^{k, \ell}\right)}^{2}\right)^{\frac{1}{2}},
$$

where $\|\cdot\|_{H_{*}^{-\frac{1}{2}}\left(\Gamma^{k, \ell}\right)}$ stands for the dual norm of $H_{00}^{\frac{1}{2}}\left(\Gamma^{k, \ell}\right)$ and

$$
\|\underline{p}\|_{-\frac{1}{2}}=\left(\sum_{k=1}^{K}\left\|p_{k}\right\|_{H^{-\frac{1}{2}}\left(\partial \Omega^{k}\right)}^{2}\right)^{\frac{1}{2}} .
$$

We also need a stability result for the Lagrange multipliers, and refer to [4] in 2D and to the annex in $3 \mathrm{D}$, in which it is proven that,

Lemma 2. There exists a constant $c_{*}$ such that, for any $p_{h, k, \ell}$ in $\tilde{W}_{h}^{k, \ell}$, there exists an element $w^{h, k, \ell}$ in $X_{h}^{k}$ that vanishes over $\partial \Omega^{k} \backslash \Gamma^{k, \ell}$ and satisfies

$$
\int_{\Gamma^{k, \ell}} p_{h, k, \ell} w^{h, k, \ell} \geq\left\|p_{h, k, \ell}\right\|_{H_{*}^{-\frac{1}{2}}\left(\Gamma^{k, \ell}\right)}^{2}
$$


with a bounded norm

$$
\left\|w^{h, k, \ell}\right\|_{H^{1}\left(\Omega^{k}\right)} \leq c_{*}\left\|p_{h, k, \ell}\right\|_{H_{*}^{-\frac{1}{2}}\left(\Gamma^{k, \ell}\right)} .
$$

Let $\pi_{k, \ell}$ denote the orthogonal projection operator from $L^{2}\left(\Gamma^{k, \ell}\right)$ onto $\tilde{W}_{h}^{k, \ell}$. Then, for $v \in L^{2}\left(\Gamma^{k, \ell}\right), \pi_{k, \ell}(v)$ is the unique element of $\tilde{W}_{h}^{k, \ell}$ such that

$$
\int_{\Gamma^{k, \ell}}\left(\pi_{k, \ell}(v)-v\right) \psi=0, \quad \forall \psi \in \tilde{W}_{h}^{k, \ell} .
$$

We are now in a position to prove the convergence of the iterative scheme

THEOREM 2. Let us assume that $\alpha h \leq c$, for some constant $c$ small enough. Then, the discrete problem (2.10) has a unique solution $\left(\underline{u}_{h}, \underline{p}_{h}\right) \in \mathcal{V}_{h}$. The algorithm (2.11)-(2.12) is well posed and converges in the sense that

$$
\lim _{n \rightarrow \infty}\left(\left\|u_{h, k}^{n}-u_{h, k}\right\|_{H^{1}\left(\Omega^{k}\right)}+\sum_{\ell \neq k}\left\|p_{h, k, \ell}^{n}-p_{h, k, \ell}\right\|_{H_{*}^{-\frac{1}{2}}\left(\Gamma^{k, \ell}\right)}\right)=0, \text { for } 1 \leq k \leq K .
$$

Proof. For the sake of convenience, we drop out the index $h$ in what follows. We first assume that problems (2.10) and (2.11)-(2.12) are well posed and proceed as in the continuous case and assume that $f=0$. From (2.15) we have

$$
\forall v_{k} \in L^{2}\left(\Gamma^{k, \ell}\right), \quad \int_{\Gamma^{k, \ell}} p_{k}^{n+1} v_{k}=\int_{\Gamma^{k, \ell}} p_{k}^{n+1} \pi_{k, \ell}\left(v_{k}\right),
$$

and (2.12) also reads

$$
p_{k}^{n+1}+\alpha \pi_{k, \ell}\left(u_{k}^{n+1}\right)=\pi_{k, \ell}\left(-p_{\ell}^{n}+\alpha u_{\ell}^{n}\right) \quad \text { over } \Gamma^{k, \ell} .
$$

By taking $v_{k}=u_{k}^{n+1}$ in (2.11), we thus have

$$
\begin{aligned}
\int_{\Omega^{k}}\left(\left|\nabla u_{k}^{n+1}\right|^{2}+\left|u_{k}^{n+1}\right|^{2}\right) d x & \\
& =\sum_{\ell} \frac{1}{4 \alpha} \int_{\Gamma^{k, \ell}}\left(\left(p_{k}^{n+1}+\alpha \pi_{k, \ell}\left(u_{k}^{n+1}\right)\right)^{2}-\left(p_{k}^{n+1}-\alpha \pi_{k, \ell}\left(u_{k}^{n+1}\right)\right)^{2}\right) d s .
\end{aligned}
$$

Then, by using the interface conditions (2.12) we obtain

$$
\begin{array}{r}
\int_{\Omega^{k}}\left(\left|\nabla u_{k}^{n+1}\right|^{2}+\left|u_{k}^{n+1}\right|^{2}\right) d x+\frac{1}{4 \alpha} \sum_{\ell} \int_{\Gamma^{k, \ell}}\left(p_{k}^{n+1}-\alpha \pi_{k, \ell}\left(u_{k}^{n+1}\right)\right)^{2} d s \\
=\frac{1}{4 \alpha} \sum_{\ell} \int_{\Gamma^{k, \ell}}\left(\pi_{k, \ell}\left(p_{\ell}^{n}-\alpha u_{\ell}^{n}\right)\right)^{2} d s .
\end{array}
$$

It is straightforward to note that

$$
\begin{aligned}
& \int_{\Gamma^{k, \ell}}\left(\pi_{k, \ell}\left(p_{\ell}^{n}-\alpha u_{\ell}^{n}\right)\right)^{2} d s \leq \int_{\Gamma^{k, \ell}}\left(p_{\ell}^{n}-\alpha u_{\ell}^{n}\right)^{2} d s \\
= & \int_{\Gamma^{k, \ell}}\left(p_{\ell}^{n}-\alpha \pi_{\ell, k}\left(u_{\ell}^{n}\right)+\alpha \pi_{\ell, k}\left(u_{\ell}^{n}\right)-\alpha u_{\ell}^{n}\right)^{2} d s \\
= & \int_{\Gamma^{k, \ell}}\left(p_{\ell}^{n}-\alpha \pi_{\ell, k}\left(u_{\ell}^{n}\right)\right)^{2}+\alpha^{2}\left(\pi_{\ell, k}\left(u_{\ell}^{n}\right)-u_{\ell}^{n}\right)^{2} d s
\end{aligned}
$$


since $\left(I d-\pi_{\ell, k}\right)\left(u_{\ell}^{n}\right)$ is orthogonal to any element in $\tilde{W}_{h}^{\ell, k}$. We then recall that (see [8] in $2 \mathrm{D}$ and [5] or [11] equation (5.1) in 3D)

$$
\begin{aligned}
\int_{\Gamma^{k, \ell}}\left(\pi_{\ell, k}\left(u_{\ell}^{n}\right)-u_{\ell}^{n}\right)^{2} d s \leq & c h\left\|u_{\ell}^{n}\right\|_{H^{1 / 2}\left(\Gamma^{k, \ell}\right)}^{2} \\
& \leq c h\left\|u_{\ell}^{n}\right\|_{H^{1}\left(\Omega^{\ell}\right)}^{2} .
\end{aligned}
$$

With similar notations as those introduced in the continuous case, we deduce

$$
E^{n+1}+B^{n+1} \leq c \alpha h E^{n}+B^{n}
$$

and we conclude as in the continuous case: if $c \alpha h<1$ then $\lim _{n \rightarrow \infty} E^{n}=0$. The convergence of $u_{k}^{n}$ towards 0 in the $H^{1}$ norm follows. The convergence of $p_{k}^{n}$ in the $H_{*}^{-\frac{1}{2}}\left(\Gamma^{k, \ell}\right)$ norm is then derived from (2.13) and (2.11). Note that by having $f=0$ and $\left(u^{n}, p^{n}\right)=0$ prove that $\left(u^{n+1}, p^{n+1}\right)=0$ from which we derive that the square problem (2.11)-(2.12) is uniquely solvable hence well posed. Similarly, having $f=0$ and getting rid of the superscripts $n$ and $n+1$ in the previous proof gives (with obvious notations) :

$$
E+B \leq c \alpha h E+B .
$$

The well posedness of (2.10) then results with similar arguments.

We shall address, in what follows this question through a more direct proof. This will allow in turn, to provide some analysis of the approximation properties of this scheme.

\section{Numerical Analysis..}

3.1. Well posedness.. The first step in this error analysis is to prove the stability of the discrete problem and thus its well posedness. Let us introduce over $\left(\prod_{k=1}^{K} H_{*}^{1}\left(\Omega^{k}\right) \times \prod_{k=1}^{K} L^{2}\left(\partial \Omega^{k}\right)\right) \times \prod_{k=1}^{K} H_{*}^{1}\left(\Omega^{k}\right)$ the bilinear form

$$
\tilde{a}((\underline{u}, \underline{p}), \underline{v}))=\sum_{k=1}^{K} \int_{\Omega^{k}}\left(\nabla u_{k} \nabla v_{k}+u_{k} v_{k}\right) d x-\sum_{k=1}^{K} \int_{\partial \Omega^{k}} p_{k} v_{k} d s .
$$

The space $\prod_{k=1}^{K} H_{*}^{1}\left(\Omega^{k}\right)$ is endowed with the norm

$$
\|\underline{v}\|_{*}=\left(\sum_{k=1}^{K}\left\|v_{k}\right\|_{H^{1}\left(\Omega^{k}\right)}^{2}\right)^{\frac{1}{2}} .
$$

LEMMA 3.

There exists a constant $\beta>0$ independent of $h$ such that

$$
\begin{aligned}
\forall\left(\underline{u}_{h}, \underline{p}_{h}\right) \in \mathcal{V}_{h}, \quad \exists \underline{v}_{h} & \in \prod_{k=1}^{K} X_{h}^{k}, \\
\left.\tilde{a}\left(\left(\underline{u}_{h}, \underline{p}_{h}\right), \underline{v}_{h}\right)\right) & \geq \beta\left(\left\|\underline{u}_{h}\right\|_{*}+\left\|\underline{p}_{h}\right\|_{-\frac{1}{2}, *}\right)\left\|\underline{v}_{h}\right\|_{*} .
\end{aligned}
$$

Moreover, we have the continuity argument : there exists a constant $c>0$ such that

$$
\begin{aligned}
\forall\left(\underline{u}_{h}, \underline{p}_{h}\right) \in \mathcal{V}_{h}, \forall \underline{v}_{h} & \in \prod_{k=1}^{K} X_{h}^{k}, \\
\left.\tilde{a}\left(\left(\underline{u}_{h}, \underline{p}_{h}\right), \underline{v}_{h}\right)\right) & \leq c\left(\left\|\underline{u}_{h}\right\|_{*}+\left\|\underline{p}_{h}\right\|_{-\frac{1}{2}}\right)\left(\left\|\underline{v}_{h}\right\|_{*}\right) .
\end{aligned}
$$


Proof of lemma 3; In (16) and (17), we have introduced local $H_{0}^{1}\left(\Gamma_{k, \ell}\right)$ functions that can be put together in order to provide an element $\underline{w}_{h}$ of $\prod_{k=1}^{K} X_{h}^{k}$ that satisfies

$$
\sum_{k=1}^{K} \int_{\partial \Omega^{k}} p_{k} w_{k} d s \geq\left\|\underline{p}_{h}\right\|_{-\frac{1}{2}, *}^{2}
$$

Let us now choose a real number $\gamma, 0<\gamma<\frac{2}{c_{*}^{2}}$ (where $c_{*}$ is introduced in (2.14) ) and choose $\underline{v}_{h}=\underline{u}_{h}-\gamma \underline{w}_{h}$ in (3.1) that yields

$$
\begin{array}{r}
\left.\tilde{a}\left(\left(\underline{u}_{h}, \underline{p}_{h}\right), \underline{v}_{h}\right)\right)=\sum_{k=1}^{K} \int_{\Omega^{k}}\left(\nabla u_{k} \nabla\left(u_{k}-\gamma w_{k}\right)+u_{k}\left(u_{k}-\gamma w_{k}\right)\right) d x \\
-\sum_{k=1}^{K} \int_{\partial \Omega^{k}} p_{k}\left(u_{k}-\gamma w_{k}\right) d s
\end{array}
$$

As already noticed in section 2.2, we can write

$$
\begin{aligned}
\int_{\Gamma^{k, \ell}} p_{k} u_{k} d s= & \frac{1}{4 \alpha} \int_{\Gamma^{k, \ell}}\left(\left(p_{k}+\alpha \pi_{k, \ell}\left(u_{k}\right)\right)^{2}-\left(p_{k}-\alpha \pi_{k, \ell}\left(u_{k}\right)\right)^{2}\right) d s \\
= & \frac{1}{4 \alpha} \int_{\Gamma^{k, \ell}}\left(\left(\pi_{k, \ell}\left(-p_{\ell}+\alpha u_{\ell}\right)\right)^{2}-\left(p_{k}-\alpha \pi_{k, \ell}\left(u_{k}\right)\right)^{2}\right) d s \\
\leq & \frac{1}{4 \alpha} \int_{\Gamma^{k, \ell}}\left(\left(p_{\ell}-\alpha u_{\ell}\right)^{2}-\left(p_{k}-\alpha \pi_{k, \ell}\left(u_{k}\right)\right)^{2}\right) d s \\
\leq & \frac{1}{4 \alpha} \int_{\Gamma^{k, \ell}}\left(\left(p_{\ell}-\alpha \pi_{\ell, k}\left(u_{\ell}\right)\right)^{2}-\left(p_{k}-\alpha \pi_{k, \ell}\left(u_{k}\right)\right)^{2}\right) d s \\
& +\frac{1}{4 \alpha} \int_{\Gamma^{k, \ell}} \alpha^{2}\left(\pi_{\ell, k}\left(u_{\ell}\right)-u_{\ell}\right)^{2} d s
\end{aligned}
$$

so that

$$
\sum_{k=1}^{K} \int_{\partial \Omega^{k}} p_{k} u_{k} d s \leq \frac{\alpha}{4} \sum_{k=1}^{K} \int_{\partial \Omega^{k}}\left(u_{k}-\pi_{k, \ell}\left(u_{k}\right)\right)^{2} d s \leq c \alpha h\left\|\underline{u}_{h}\right\|_{*}^{2} .
$$

Going back to (3.4) yields

$$
\begin{aligned}
\tilde{a}\left(\left(\underline{u}_{h}, \underline{p}_{h}\right), \underline{v}_{h}\right) & \geq(1-c \alpha h)\left\|\underline{u}_{h}\right\|_{*}^{2}-\gamma\left\|\underline{u}_{h}\right\|_{*}\left\|\underline{w}_{h}\right\|_{*}+\gamma\left\|\underline{p}_{h}\right\|_{-\frac{1}{2}, *}^{2} \\
& \geq\left(\frac{1}{2}-c \alpha h\right)\left\|\underline{u}_{h}\right\|_{*}^{2}+\gamma\left\|\underline{p}_{h}\right\|_{-\frac{1}{2}, *}^{2}-\frac{\gamma^{2}}{2}\left\|\underline{w}_{h}\right\|_{*}^{2} \\
& \geq\left(\frac{1}{2}-c \alpha h\right)\left\|\underline{u}_{h}\right\|_{*}^{2}+\left(\gamma-\frac{\gamma^{2} c_{*}^{2}}{2}\right)\left\|\underline{p}_{h}\right\|_{-\frac{1}{2}, *}^{2} \cdot
\end{aligned}
$$

Due to the choice of $\gamma$, we know that, for $\alpha h$ small enough, (3.2) holds. The continuity (3.3) follows from standard arguments (note that the norm on the right hand side of (3.3) is not the $\|\cdot\|_{-\frac{1}{2}, *}$-norm), which ends the proof of lemma 3 .

From this lemma, we have the following result :

THEOREM 3. Under the hypothesis of theorem 2, the discrete problem (2.10) has a unique solution $\left(\underline{u}_{h}, \underline{p}_{h}\right) \in \mathcal{V}_{h}$, and there exists a constant $c>0$ such that

$$
\left\|\underline{u}_{h}\right\|_{*}+\left\|\underline{p}_{h}\right\|_{-\frac{1}{2}, *} \leq c\|f\|_{L^{2}(\Omega)} .
$$


From lemma 3, we are also in position to state that for any $\left(\underline{\tilde{u}}_{h}, \underline{\tilde{p}}_{h}\right) \in \mathcal{V}_{h}$,

$$
\left\|\underline{u}-\underline{u}_{h}\right\|_{*}+\left\|\underline{p}-\underline{p}_{h}\right\|_{-\frac{1}{2}, *} \leq c\left(\left\|\underline{u}-\underline{\tilde{u}}_{h}\right\|_{*}+\left\|\underline{p}-\underline{\tilde{p}}_{h}\right\|_{-\frac{1}{2}}\right)
$$

and we are naturally led to the analysis of the best fit of $(\underline{u}, \underline{p})$ by elements in $\mathcal{V}_{h}$.

3.2. Analysis of the best fit in 2D. In this part we analyze the best approximation of $(\underline{u}, \underline{p})$ by elements in $\mathcal{V}_{h}$. As the proof is very technical for the analysis of the best fit, we restrict ourselves in this section to the complete analysis of the $2 \mathrm{D}$ situation. The extension to $3 \mathrm{D}$ first order approximation is postponed to the next subsection.

The first step in the analysis is to prove the following lemma

Lemma 4. Assume the degree of the finite element approximation $M \leq 13$, there exists two constants $c_{1}>0$ and $c_{2}>0$ independent of $h$ such that for all $\eta_{\ell, k}$ in $\mathcal{Y}_{h}^{\ell, k} \cap H_{0}^{1}\left(\Gamma^{k, \ell}\right)$, there exists an element $\psi_{\ell, k}$ in $\tilde{W}_{h}^{\ell, k}$, such that

$$
\begin{gathered}
\int_{\Gamma^{k, \ell}}\left(\eta_{\ell, k}+\pi_{k, \ell}\left(\eta_{\ell, k}\right)\right) \psi_{\ell, k} \geq c_{1}\left\|\eta_{\ell, k}\right\|_{L^{2}\left(\Gamma^{k, \ell}\right)}^{2}, \\
\left\|\psi_{\ell, k}\right\|_{L^{2}\left(\Gamma^{k, \ell}\right)} \leq c_{2}\left\|\eta_{\ell, k}\right\|_{L^{2}\left(\Gamma^{k, \ell}\right)} .
\end{gathered}
$$

Then, we can prove the following interpolation estimates :

THEOREM 4. For any $u \in H^{2}(\Omega) \cap H_{0}^{1}(\Omega)$, such that $u_{k}=u_{\mid \Omega^{k}} \in H^{2+m}\left(\Omega^{k}\right)$, $1 \leq k \leq K$ with $M-1 \geq m \geq 0, \underline{u}=\left(u_{k}\right)_{1 \leq k \leq K}$ and let $p_{k, \ell}=\frac{\partial u}{\partial \mathbf{n}_{k}}$ over each $\Gamma^{k, \ell}$. Then there exists an element $\underline{\tilde{\tilde{u}}}_{h}$ in $\prod_{k=1}^{K} X_{h}^{k}$ and $\underline{\tilde{p}}_{h}=\left(\tilde{p}_{k \ell h}\right), \tilde{p}_{k \ell h} \in \tilde{W}_{h}^{k, \ell}$ such that $\left(\underline{\tilde{u}}_{h}, \underline{\tilde{p}}_{h}\right)$ satisfy the coupling condition (2.9), and

$$
\begin{aligned}
\left\|\underline{\tilde{u}}_{h}-\underline{u}\right\|_{*} \leq & c h^{1+m} \sum_{k=1}^{K}\left\|u_{k}\right\|_{H^{2+m}\left(\Omega^{k}\right)}+\frac{c h^{m}}{\alpha} \sum_{k<\ell}\left\|p_{k, \ell}\right\|_{H^{\frac{1}{2}+m}\left(\Gamma^{k, \ell}\right)} \\
\left\|\tilde{p}_{k \ell h}-p_{k, \ell}\right\|_{H^{-\frac{1}{2}}\left(\Gamma^{k, \ell}\right)} \leq & c \alpha h^{2+m}\left(\left\|u_{k}\right\|_{H^{2+m}\left(\Omega^{k}\right)}+\left\|u_{\ell}\right\|_{H^{2+m}\left(\Omega^{\ell}\right)}\right) \\
& +c h^{1+m}\left\|p_{k, \ell}\right\|_{H^{\frac{1}{2}+m}\left(\Gamma^{k, \ell}\right)}
\end{aligned}
$$

where $c$ is a constant independent of $h$ and $\alpha$. If we assume more regularity on the normal derivatives on the interfaces, we have

Theorem 5. Let $u \in H^{2}(\Omega) \cap H_{0}^{1}(\Omega)$, such that $u_{k}=u_{\mid \Omega^{k}} \in H^{2+m}\left(\Omega^{k}\right)$, $1 \leq k \leq K$ with $M-1 \geq m \geq 0, \underline{u}=\left(u_{k}\right)_{1 \leq k \leq K}$ and $p_{k, \ell}=\frac{\partial u}{\partial \mathbf{n}_{k}}$ is in $H^{\frac{3}{2}+m}\left(\Gamma_{k, \ell}\right)$. Then there exists $\underline{\tilde{u}}_{h}$ in $\prod_{k=1}^{K} X_{h}^{k}$ and $\underline{\tilde{x}}_{h}=\left(\tilde{p}_{k \ell h}\right)$, $\tilde{p}_{k \ell h} \in \tilde{W}_{h}^{k, \ell}$ such that $\left(\underline{\tilde{\tilde{x}}}_{h}, \underline{\tilde{p}}_{h}\right)$ satisfy (2.9), and

$$
\begin{aligned}
\left\|\underline{\tilde{u}}_{h}-\underline{u}\right\|_{*} \leq & c h^{1+m} \sum_{k=1}^{K}\left\|u_{k}\right\|_{H^{2+m}\left(\Omega^{k}\right)} \\
& +\frac{c h^{m+1}}{\alpha}(\log h)^{\beta(m)} \sum_{k<\ell}\left\|p_{k, \ell}\right\|_{H^{\frac{3}{2}+m}\left(\Gamma^{k, \ell}\right)} \\
\left\|\tilde{p}_{k \ell h}-p_{k, \ell}\right\|_{H^{-\frac{1}{2}}\left(\Gamma^{k, \ell}\right)} \leq & c \alpha h^{2+m}\left(\left\|u_{k}\right\|_{H^{2+m}\left(\Omega^{k}\right)}+\left\|u_{\ell}\right\|_{H^{2+m}\left(\Omega^{\ell}\right)}\right) \\
& +c h^{1+m}(\log h)^{\beta(m)}\left\|p_{k, \ell}\right\|_{H^{\frac{1}{2}+m}\left(\Gamma^{k, \ell}\right)}
\end{aligned}
$$


where $c$ is a constant independent of $h$ and $\alpha$, and $\beta(m)=0$ if $m \leq M-2$ and $\beta(m)=1$ if $m=M-1$.

Proof of lemma 4: We first give the proof for $P_{1}$ finite element. The general proof is quite technical and is based on Lemma 8, given in Appendix B. Remind that we have denoted as $x_{0}^{\ell, k}, x_{1}^{\ell, k}, \ldots, x_{n-1}^{\ell, k}, x_{n}^{\ell, k}$ the vertices of the triangulation of $\Gamma^{\ell, k}$ that belong to $\Gamma^{\ell, k}$. To any $\eta_{\ell, k}$ in $\mathcal{Y}_{h}^{\ell, k} \cap H_{0}^{1}\left(\Gamma^{k, \ell}\right)$ we then associate the element $\psi_{\ell, k}$ in $\tilde{W}_{h}^{\ell, k}$ as follows

$$
\psi_{\ell, k}=\left\{\begin{array}{l}
\left.\frac{\eta_{\ell, k}\left(x_{1}^{\ell, k}-x_{0}^{\ell, k}\right)}{\left(x-x_{0}^{\ell, k}\right)} \text { over }\right] x_{0}^{\ell, k}, x_{1}^{\ell, k}[ \\
\left.\eta_{\ell, k} \text { over }\right] x_{1}^{\ell, k}, x_{n-1}^{\ell, k}[ \\
\left.\frac{\eta_{\ell, k}\left(x_{n}^{\ell, k}-x_{n-1}^{\ell, k}\right)}{\left(x_{n}^{\ell, k}-x\right)} \text { over }\right] x_{n-1}^{\ell, k}, x_{n}^{\ell, k}[
\end{array}\right.
$$

recalling that all norms are equivalent over the space of polynomials of degree 1 we easily deduce that there exists a constant $c$ such that

$$
\left\|\psi_{\ell, k}\right\|_{L^{2}\left(\Gamma^{k, \ell}\right)} \leq c\left\|\eta_{\ell, k}\right\|_{L^{2}\left(\Gamma^{k, \ell}\right)} .
$$

Moreover, it is straightforward to derive

$$
\begin{aligned}
\int_{\Gamma^{k, \ell}}\left(\eta_{\ell, k}+\pi_{k, \ell}\left(\eta_{\ell, k}\right)\right) \psi_{\ell, k}= & \int_{\Gamma^{k, \ell}} \eta_{\ell, k} \psi_{\ell, k}+\int_{\Gamma^{k, \ell}}\left(\pi_{k, \ell}\left(\eta_{\ell, k}\right)\right)^{2} \\
& +\int_{\Gamma^{k, \ell}} \pi_{k, \ell}\left(\eta_{\ell, k}\right)\left(\psi_{\ell, k}-\eta_{\ell, k}\right) .
\end{aligned}
$$

Then, by using the relation

$$
\pi_{k, \ell}\left(\eta_{\ell, k}\right)\left(\psi_{\ell, k}-\eta_{\ell, k}\right) \geq-\frac{1}{2}\left(\pi_{k, \ell}\left(\eta_{\ell, k}\right)\right)^{2}-\frac{1}{2}\left(\psi_{\ell, k}-\eta_{\ell, k}\right)^{2}
$$

leads to

$$
\int_{\Gamma^{k, \ell}}\left(\eta_{\ell, k}+\pi_{k, \ell}\left(\eta_{\ell, k}\right)\right) \psi_{\ell, k} \geq \int_{\Gamma^{k, \ell}} \eta_{\ell, k} \psi_{\ell, k}+\frac{1}{2} \int_{\Gamma^{k, \ell}}\left(\pi_{k, \ell}\left(\eta_{\ell, k}\right)\right)^{2}-\frac{1}{2} \int_{\Gamma^{k, \ell}}\left(\psi_{\ell, k}-\eta_{\ell, k}\right)^{2} .
$$

We realize now that, over the first interval,

$$
\int_{]_{0}^{\ell, k}, x_{1}^{\ell, k}[}\left(\eta_{\ell, k} \psi_{\ell, k}-\frac{1}{2}\left(\psi_{\ell, k}-\eta_{\ell, k}\right)^{2}\right)=\int_{] x_{0}^{\ell, k}, x_{1}^{\ell, k}[}\left(\frac{\left(x-x_{0}^{\ell, k}\right)}{\left(x_{1}^{\ell, k}-x_{0}^{\ell, k}\right)}-\frac{1}{2} \frac{\left(x-x_{1}^{\ell, k}\right)^{2}}{\left(x_{1}^{\ell, k}-x_{0}^{\ell, k}\right)^{2}}\right) \psi_{\ell, k}^{2}
$$

noticing that

$$
\int_{] x_{0}^{\ell, k}, x_{1}^{\ell, k}[}\left(\frac{\left(x-x_{0}^{\ell, k}\right)}{\left(x_{1}^{\ell, k}-x_{0}^{\ell, k}\right)}-\frac{1}{2} \frac{\left(x-x_{1}^{\ell, k}\right)^{2}}{\left(x_{1}^{\ell, k}-x_{0}^{\ell, k}\right)^{2}}\right)=\int_{] x_{0}^{\ell, k}, x_{1}^{\ell, k}[} \frac{\left(x-x_{0}^{\ell, k}\right)^{2}}{\left(x_{1}^{\ell, k}-x_{0}^{\ell, k}\right)^{2}},
$$

by recalling that $\psi_{\ell, k}$ is constant on $] x_{0}^{\ell, k}, x_{1}^{\ell, k}[$ and $] x_{n-1}^{\ell, k}, x_{n}^{\ell, k}[$, we derive that

$$
\int_{\Gamma^{k, \ell}}\left(\eta_{\ell, k}+\pi_{k, \ell}\left(\eta_{\ell, k}\right)\right) \psi_{\ell, k} \geq \int_{\Gamma^{k, \ell}} \eta_{\ell, k}^{2}
$$

which ends the proof of lemma 4 
PROOF of Lemma 4 in the general case Using the definition of $\pi_{k, \ell}$, (2.15), it is straightforward to derive

$$
\begin{aligned}
\int_{\Gamma^{k, \ell}}\left(\eta_{\ell, k}+\pi_{k, \ell}\left(\eta_{\ell, k}\right)\right) \psi_{\ell, k}= & \int_{\Gamma^{k, \ell}} \eta_{\ell, k} \psi_{\ell, k}+\int_{\Gamma^{k, \ell}}\left(\pi_{k, \ell}\left(\eta_{\ell, k}\right)\right)^{2} \\
& +\int_{\Gamma^{k, \ell}} \pi_{k, \ell}\left(\eta_{\ell, k}\right)\left(\psi_{\ell, k}-\eta_{\ell, k}\right) .
\end{aligned}
$$

Then, using the relation

$$
\pi_{k, \ell}\left(\eta_{\ell, k}\right)\left(\psi_{\ell, k}-\eta_{\ell, k}\right) \geq-\left(\pi_{k, \ell}\left(\eta_{\ell, k}\right)\right)^{2}-\frac{1}{4}\left(\psi_{\ell, k}-\eta_{\ell, k}\right)^{2}
$$

leads to

$$
\int_{\Gamma^{k, \ell}}\left(\eta_{\ell, k}+\pi_{k, \ell}\left(\eta_{\ell, k}\right)\right) \psi_{\ell, k} \geq \int_{\Gamma^{k, \ell}} \eta_{\ell, k} \psi_{\ell, k}-\frac{1}{4} \int_{\Gamma^{k, \ell}}\left(\psi_{\ell, k}-\eta_{\ell, k}\right)^{2} .
$$

Remind that we have denoted as $x_{0}^{\ell, k}, x_{1}^{\ell, k}, \ldots, x_{n-1}^{\ell, k}, x_{n}^{\ell, k}$ the vertices of the triangulation of $\Gamma^{\ell, k}$ that belong to $\Gamma^{\ell, k}$. By Lemma 8 of appendix B, and an easy scaling argument, there exists $c, C>0, \psi_{1}$ from $\left[x_{0}^{\ell, k}, x_{1}^{\ell, k}\right]$ into $\mathbb{R}$ and $\psi_{n}$ from $\left[x_{n-1}^{\ell, k}, x_{n}^{\ell, k}\right]$ into $\mathbb{R}$ such that

$$
\begin{gathered}
\left\|\psi_{1}\right\|_{L^{2}\left(x_{0}^{\ell, k}, x_{1}^{\ell, k}\right)}+\left\|\psi_{n}\right\|_{L^{2}\left(x_{n-1}^{\ell, k}, x_{n}^{\ell, k}\right)} \leq C^{2}\left(\|\eta\|_{L^{2}\left(x_{0}^{\ell, k}, x_{1}^{\ell, k}\right)}+\|\eta\|_{L^{2}\left(x_{n-1}^{\ell, k}, x_{n}^{\ell, k}\right)}\right), \\
\psi_{1}\left(x_{1}^{\ell, k}\right)=\eta\left(x_{1}^{\ell, k}\right), \psi_{N}\left(x_{n-1}^{\ell, k}\right)=\eta\left(x_{n-1}^{\ell, k}\right) \text { and } \\
\int_{x_{0}^{\ell, k}}^{x_{1}^{\ell, k}}\left(\eta \psi_{1}-\frac{1}{4}\left(\psi_{1}-\eta\right)^{2}\right)+\int_{x_{n-1}^{\ell, k}}^{x_{n}^{\ell, k}}\left(\eta \psi_{n}-\frac{1}{4}\left(\psi_{n}-\eta\right)^{2}\right) \geq c\left(\int_{x_{0}^{\ell, k}}^{x_{1}^{\ell, k}} \eta^{2}+\int_{x_{n-1}^{\ell, k}}^{x_{n}^{\ell, k}} \eta^{2}\right) .
\end{gathered}
$$

Taking $\psi_{\ell, k}$ in $\tilde{W}_{h}^{\ell, k}$ as follows

$$
\psi_{\ell, k}=\left\{\begin{array}{l}
\left.\psi_{1} \text { over }\right] x_{0}^{\ell, k}, x_{1}^{\ell, k}[ \\
\left.\eta_{\ell, k} \text { over }\right] x_{1}^{\ell, k}, x_{n-1}^{\ell, k}[ \\
\left.\psi_{n} \text { over }\right] x_{n-1}^{\ell, k}, x_{n}^{\ell, k}[
\end{array}\right.
$$

proves Lemma 4 with $c_{1}=\min (1, c)$ and $c_{2}=\max (1, C)$.

Proof of theorem 4; In order to prove this theorem, let us build an element that will belong to the discrete space and will be as close as the expected error to the solution. Let $u_{k h}^{1}$ be the unique element of $X_{h}^{k}$ defined as follows :

- $\left(u_{k h}^{1}\right)_{\mid \partial \Omega^{k}}$ is the best fit of $u_{k}$ over $\partial \Omega^{k}$ in $\mathcal{Y}_{h}^{k, \ell}$,

- $u_{k h}^{1}$ at the inner nodes of the triangulation (in $\Omega^{k}$ ) coincide with the interpolate of $u_{k}$.

Then, it satisfies

$$
\left\|u_{k h}^{1}-u_{k}\right\|_{L^{2}\left(\partial \Omega^{k}\right)} \leq c h^{\frac{3}{2}+m}\left\|u_{k}\right\|_{H^{2+m}\left(\Omega^{k}\right)},
$$

from which we deduce that

$$
\left\|u_{k h}^{1}-u_{k}\right\|_{L^{2}\left(\Omega^{k}\right)}+h\left\|u_{k h}^{1}-u_{k}\right\|_{H^{1}\left(\Omega^{k}\right)} \leq c h^{2+m}\left\|u_{k}\right\|_{H^{2+m}\left(\Omega^{k}\right)},
$$


and, from Aubin-Nitsche estimate

$$
\left\|u_{k h}^{1}-u_{k}\right\|_{H^{-\frac{1}{2}}\left(\Gamma^{k, \ell}\right)} \leq c h^{2+m}\left\|u_{k}\right\|_{H^{2+m}\left(\Omega^{k}\right)} .
$$

We define then separately the best fit $p_{k \ell h}^{1}$ of $p_{k, \ell}=\frac{\partial u}{\partial \mathbf{n}_{k}}$ over each $\Gamma^{k, \ell}$ in $\tilde{W}_{h}^{k, \ell}$. These elements satisfy for $0 \leq m \leq M-1$ the error estimate

$$
\begin{aligned}
\left\|p_{k \ell h}^{1}-p_{k, \ell}\right\|_{L^{2}\left(\Gamma^{k, \ell}\right)} & \leq c h^{\frac{1}{2}+m}\left\|p_{k, \ell}\right\|_{H^{\frac{1}{2}+m}\left(\Gamma^{k, \ell}\right)} \\
\left\|p_{k \ell h}^{1}-p_{k, \ell}\right\|_{H^{-\frac{1}{2}}\left(\Gamma^{k, \ell}\right)} & \leq c h^{1+m}\left\|p_{k, \ell}\right\|_{H^{\frac{1}{2}+m}\left(\Gamma^{k, \ell}\right)} .
\end{aligned}
$$

But there is very few chance that $\left(\underline{u}_{h}^{1}, \underline{p}_{h}^{1}\right)$ satisfy the coupling condition (2.9). This element of $\left(\prod_{k=1}^{K} X_{h}^{k}\right) \times\left(\prod_{k=1}^{K} \tilde{W}_{h}^{k}\right)$ misses (2.9) of elements $\epsilon_{k, \ell}$ and $\eta_{\ell, k}$ such that

$$
\begin{aligned}
& \int_{\Gamma^{k, \ell}}\left(p_{k \ell h}^{1}+\epsilon_{k, \ell}+\alpha u_{k h}^{1}\right) \psi_{k, \ell}=\int_{\Gamma^{k, \ell}}\left(-p_{\ell k h}^{1}+\alpha \eta_{\ell, k}+\alpha u_{\ell h}^{1}\right) \psi_{k, \ell}, \forall \psi_{k, \ell} \in \tilde{W}_{h}^{k, \ell} \\
& \int_{\Gamma^{k, \ell}}\left(p_{\ell k h}^{1}+\alpha \eta_{\ell, k}+\alpha u_{\ell h}^{1}\right) \psi_{\ell, k}=\int_{\Gamma^{k, \ell}}\left(-p_{k \ell h}^{1}-\epsilon_{k, \ell}+\alpha u_{k h}^{1}\right) \psi_{\ell, k}, \forall \psi_{\ell, k} \in \tilde{W}_{h}^{\ell, k}
\end{aligned}
$$

In order to correct that, without polluting (3.8)-(3.12), for each couple $(k, \ell)$ we choose one side, say the smaller indexed one, hereafter we shall also assume that each couple $(k, \ell)$ is ordered by $k<\ell$. Associated to that choice, we define $\epsilon_{k, \ell} \in \tilde{W}_{h}^{k, \ell}$, $\eta_{\ell, k} \in \mathcal{Y}_{h}^{\ell, k} \cap H_{0}^{1}\left(\Gamma^{k, \ell}\right)$, such that $\left(\underline{\tilde{\tilde{x}}}_{h}, \underline{\tilde{p}}_{h}\right)$ satisfy (2.9) where we define

$$
\begin{gathered}
\tilde{u}_{\ell h}=u_{\ell h}^{1}+\sum_{k<\ell} \mathcal{R}_{\ell, k}\left(\eta_{\ell, k}\right) \\
\tilde{p}_{k \ell h}=p_{k \ell h}^{1}+\epsilon_{k, \ell} \quad(\text { for } k<\ell)
\end{gathered}
$$

where $\mathcal{R}_{\ell, k}$ is a discrete lifting operator (see [36], [7]) that to any element of $\mathcal{Y}_{h}^{\ell, k} \cap$ $H_{0}^{1}\left(\Gamma^{k, \ell}\right)$ associates a finite element function over $\Omega^{\ell}$ that vanishes over $\partial \Omega^{\ell} \backslash \Gamma^{k, \ell}$ and satisfies

$$
\begin{array}{r}
\forall w \in \mathcal{Y}_{h}^{\ell, k} \cap H_{0}^{1}\left(\Gamma^{k, \ell}\right), \quad\left(\mathcal{R}_{\ell, k}(w)\right)_{\mid \Gamma_{k, \ell}}=w \\
\left\|\mathcal{R}_{\ell, k}(w)\right\|_{H^{1}\left(\Omega^{\ell}\right)} \leq c\|w\|_{H_{00}^{\frac{1}{2}}\left(\Gamma^{k, \ell}\right)}
\end{array}
$$

where $c$ is $h$-independent.

The set of equations (3.13)-(3.14) for $\epsilon_{k, \ell}$ and $\eta_{\ell, k}$ results in a square system of linear algebraic equations that can be written as follows

$$
\begin{gathered}
\int_{\Gamma^{k, \ell}}\left(\epsilon_{k, \ell}-\alpha \eta_{\ell, k}\right) \psi_{k, \ell}=\int_{\Gamma^{k, \ell}} e_{1} \psi_{k, \ell}, \forall \psi_{k, \ell} \in \tilde{W}_{h}^{k, \ell} \\
\int_{\Gamma^{k, \ell}}\left(\epsilon_{k, \ell}+\alpha \eta_{\ell, k}\right) \psi_{\ell, k}=\int_{\Gamma^{k, \ell}} e_{2} \psi_{\ell, k}, \forall \psi_{\ell, k} \in \tilde{W}_{h}^{\ell, k}
\end{gathered}
$$


with

$$
e_{1}=-p_{k \ell h}^{1}-p_{\ell k h}^{1}+\alpha\left(u_{\ell h}^{1}-u_{k h}^{1}\right)
$$

and

$$
e_{2}=-p_{k \ell h}^{1}-p_{\ell k h}^{1}+\alpha\left(u_{k h}^{1}-u_{\ell h}^{1}\right)
$$

Proposition 3.1. The linear system (3.17)-(3.18) is well posed.

Proof: With these notations, (3.17) yields

$$
\epsilon_{k, \ell}=\pi_{k, \ell}\left(\alpha \eta_{\ell, k}+e_{1}\right)
$$

and (3.18) yields

$$
\alpha \eta_{\ell, k}=\pi_{\ell, k}\left(-\epsilon_{k, \ell}+e_{2}\right) .
$$

As (3.17)-(3.18) is a square linear system, it suffices to prove uniqueness for $e_{1}$ and $e_{2}$ null. From (3.21)-(3.22), we get

$$
0=\eta_{\ell, k}+\pi_{\ell, k} \pi_{k, \ell}\left(\eta_{\ell, k}\right) .
$$

so that for all $\psi_{\ell, k}$ in $\tilde{W}_{h}^{k, \ell}$,

$$
0=\int_{\Gamma^{k, \ell}}\left(\eta_{\ell, k}+\pi_{k, \ell}\left(\eta_{\ell, k}\right)\right) \psi_{\ell, k}
$$

By Lemma 4, this proves that $\eta_{\ell, k}$ is zero, thus by (3.21), $\epsilon_{k, \ell}$ is zero.

Let us resume the proof of theorem 4 By (3.21) and (3.22) we have

$$
\int_{\Gamma^{k, \ell}}\left(\eta_{\ell, k}+\pi_{k, \ell}\left(\eta_{\ell, k}\right)\right) \psi_{\ell, k}=\frac{1}{\alpha} \int_{\Gamma^{k, \ell}}\left(e_{2}-\pi_{k, \ell}\left(e_{1}\right)\right) \psi_{\ell, k}, \forall \psi_{\ell, k} \in \tilde{W}_{h}^{\ell, k} .
$$

In order to estimate $\left\|\tilde{p}_{k \ell h}-p_{k, \ell}\right\|_{H^{-\frac{1}{2}\left(\Gamma^{k, \ell}\right)}}$ and $\left\|\tilde{u}_{\ell h}-u_{\ell}\right\|_{H^{1}\left(\Omega^{\ell}\right)}$, we first estimate $\left\|\eta_{\ell, k}\right\|_{L^{2}\left(\Gamma^{k, \ell}\right)}$ :

from (3.6) and (3.23) we get

$$
c_{1}\left\|\eta_{\ell, k}\right\|_{L^{2}\left(\Gamma^{k, \ell}\right)}^{2} \leq \frac{1}{\alpha}\left\|e_{2}-\pi_{k, \ell}\left(e_{1}\right)\right\|_{L^{2}\left(\Gamma^{k, \ell}\right)}\left\|\psi_{\ell, k}\right\|_{L^{2}\left(\Gamma^{k, \ell}\right)}
$$

and using (3.7) in (3.24)

$$
\left\|\eta_{\ell, k}\right\|_{L^{2}\left(\Gamma^{k, \ell}\right)} \leq \frac{c_{2}}{\alpha c_{1}}\left\|e_{2}-\pi_{k, \ell}\left(e_{1}\right)\right\|_{L^{2}\left(\Gamma^{k, \ell}\right)}
$$

hence

$$
\left\|\eta_{\ell, k}\right\|_{L^{2}\left(\Gamma^{k, \ell}\right)} \leq \frac{c_{2}}{\alpha c_{1}}\left(\left\|e_{2}\right\|_{L^{2}\left(\Gamma^{k, \ell}\right)}+\left\|e_{1}\right\|_{L^{2}\left(\Gamma^{k, \ell}\right)}\right)
$$

Now, from (3.19) and (3.20), for $i=1,2$

$$
\left\|e_{i}\right\|_{L^{2}\left(\Gamma^{k, \ell}\right)} \leq\left\|p_{k \ell h}^{1}+p_{\ell k h}^{1}\right\|_{L^{2}\left(\Gamma^{k, \ell}\right)}+\alpha\left\|u_{\ell h}^{1}-u_{k h}^{1}\right\|_{L^{2}\left(\Gamma^{k, \ell}\right)}
$$


and recalling that $p_{k, \ell}=\frac{\partial u}{\partial \mathbf{n}_{k}}=-\frac{\partial u}{\partial \mathbf{n}_{\ell}}=-p_{\ell, k}$ over each $\Gamma^{k, \ell}$

$$
\begin{aligned}
\left\|p_{k \ell h}^{1}+p_{\ell k h}^{1}\right\|_{L^{2}\left(\Gamma^{k, \ell}\right)} & \leq\left\|p_{k \ell h}^{1}-p_{k, \ell}\right\|_{L^{2}\left(\Gamma^{k, \ell}\right)}+\left\|p_{\ell k h}^{1}-p_{\ell, k}\right\|_{L^{2}\left(\Gamma^{k, \ell}\right)} \\
\left\|u_{\ell h}^{1}-u_{k h}^{1}\right\|_{L^{2}\left(\Gamma^{k, \ell}\right)} & \leq\left\|u_{k h}^{1}-u_{k}\right\|_{L^{2}\left(\Gamma^{k, \ell}\right)}+\left\|u_{\ell h}^{1}-u_{\ell}\right\|_{L^{2}\left(\Gamma^{k, \ell}\right)}
\end{aligned}
$$

so that, using (3.8) and (3.11), we derive for $i=1,2$ and $0 \leq m \leq M-1$

$\left\|e_{i}\right\|_{L^{2}\left(\Gamma^{k, \ell}\right)} \leq c \alpha h^{\frac{3}{2}+m}\left(\left\|u_{k}\right\|_{H^{2+m}\left(\Omega^{k}\right)}+\left\|u_{\ell}\right\|_{H^{2+m}\left(\Omega^{\ell}\right)}\right)+c h^{\frac{1}{2}+m}\left\|p_{k, \ell}\right\|_{H^{\frac{1}{2}+m}\left(\Gamma^{k, \ell}\right)}$

and (3.25) yields for $0 \leq m \leq M-1$

$\left\|\eta_{\ell, k}\right\|_{L^{2}\left(\Gamma^{k, \ell}\right)} \leq \operatorname{ch} h^{\frac{3}{2}+m}\left(\left\|u_{k}\right\|_{H^{2+m}\left(\Omega^{k}\right)}+\left\|u_{\ell}\right\|_{H^{2+m}\left(\Omega^{\ell}\right)}\right)+\frac{c h^{\frac{1}{2}+m}}{\alpha}\left\|p_{k, \ell}\right\|_{H^{\frac{1}{2}+m}\left(\Gamma^{k, \ell}\right)}$.

We can now evaluate $\left\|\tilde{p}_{k \ell h}-p_{k, \ell}\right\|_{H^{-\frac{1}{2}\left(\Gamma^{k, \ell}\right)}}$, using (3.15) :

$$
\left\|\tilde{p}_{k \ell h}-p_{k, \ell}\right\|_{H^{-\frac{1}{2}}\left(\Gamma^{k, \ell}\right)} \leq\left\|\epsilon_{k, \ell}\right\|_{H^{-\frac{1}{2}\left(\Gamma^{k, \ell}\right)}}+\left\|p_{k \ell h}^{1}-p_{k, \ell}\right\|_{H^{-\frac{1}{2}}\left(\Gamma^{k, \ell}\right)} \text {. }
$$

The term $\left\|p_{k \ell h}^{1}-p_{k, \ell}\right\|_{H^{-\frac{1}{2}}\left(\Gamma^{k, \ell}\right)}$ is estimated in (3.12), so let us focus on the term $\left\|\epsilon_{k, \ell}\right\|_{H^{-\frac{1}{2}\left(\Gamma^{k, \ell}\right)}}$. From (3.21) we have,

$$
\left\|\epsilon_{k, \ell}\right\|_{H^{-\frac{1}{2}\left(\Gamma^{k, \ell}\right)}} \leq \alpha\left\|\eta_{\ell, k}\right\|_{H^{-\frac{1}{2}\left(\Gamma^{k, \ell}\right)}}+\left\|e_{1}\right\|_{H^{-\frac{1}{2}\left(\Gamma^{k, \ell}\right)}}+\left\|\left(I d-\pi_{k, \ell}\right)\left(\alpha \eta_{\ell, k}+e_{1}\right)\right\|_{H^{-\frac{1}{2}\left(\Gamma^{k, \ell}\right)}} \text {. }
$$

To evaluate $\left\|e_{1}\right\|_{H^{-\frac{1}{2}\left(\Gamma^{k, \ell}\right)}}$ we proceed as for $\left\|e_{1}\right\|_{L^{2}\left(\Gamma^{k, \ell}\right)}$ and from (3.10) and (3.12) we have, for $i=1,2$ and $0 \leq m \leq M$ :

$\left\|e_{i}\right\|_{H^{-\frac{1}{2}}\left(\Gamma^{k, \ell}\right)} \leq c \alpha h^{2+m}\left(\left\|u_{k}\right\|_{H^{2+m}\left(\Omega^{k}\right)}+\left\|u_{\ell}\right\|_{H^{2+m}\left(\Omega^{\ell}\right)}\right)+c h^{1+m}\left\|p_{k, \ell}\right\|_{H^{\frac{1}{2}+m}\left(\Gamma^{k, \ell}\right)} \cdot$

The third term in the right member of (3.29) satisfies

$$
\left\|\left(I d-\pi_{k, \ell}\right)\left(\alpha \eta_{\ell, k}+e_{1}\right)\right\|_{H^{-\frac{1}{2}}\left(\Gamma^{k, \ell}\right)} \leq c \sqrt{h}\left\|\alpha \eta_{\ell, k}+e_{1}\right\|_{L^{2}\left(\Gamma^{k, \ell}\right)} .
$$

Then, using (3.27) and (3.26) yields

$$
\begin{aligned}
\left\|\left(I d-\pi_{k, \ell}\right)\left(\alpha \eta_{\ell, k}+e_{1}\right)\right\|_{H^{-\frac{1}{2}\left(\Gamma^{k, \ell}\right)}} \leq & c \alpha h^{2+m}\left(\left\|u_{k}\right\|_{H^{2+m}\left(\Omega^{k}\right)}+\left\|u_{\ell}\right\|_{H^{2+m}\left(\Omega^{\ell}\right)}\right) \\
& +c h^{1+m}\left\|p_{k, \ell}\right\|_{H^{\frac{1}{2}+m}\left(\Gamma^{k, \ell}\right)}
\end{aligned}
$$

In order to estimate the term $\left\|\eta_{\ell, k}\right\|_{H^{-\frac{1}{2}\left(\Gamma^{k, \ell}\right)}}$ in (3.29), we use (3.23):

$$
\begin{aligned}
2 \int_{\Gamma^{k, \ell}} \eta_{\ell, k} \psi_{\ell, k} & =2 \int_{\Gamma^{k, \ell}} \eta_{\ell, k} \psi_{\ell, k} \\
& -\left(\int_{\Gamma^{k, \ell}}\left(\eta_{\ell, k}+\pi_{k, \ell}\left(\eta_{\ell, k}\right)\right) \psi_{\ell, k}-\frac{1}{\alpha} \int_{\Gamma^{k, \ell}}\left(e_{2}-\pi_{k, \ell}\left(e_{1}\right)\right) \psi_{\ell, k}\right)
\end{aligned}
$$

that is

$$
2 \int_{\Gamma^{k, \ell}} \eta_{\ell, k} \psi_{\ell, k}=\int_{\Gamma^{k, \ell}}\left(\eta_{\ell, k}-\pi_{k, \ell} \eta_{\ell, k}\right) \psi_{\ell, k}+\frac{1}{\alpha} \int_{\Gamma^{k, \ell}}\left(e_{2}-\pi_{k, \ell}\left(e_{1}\right)\right) \psi_{\ell, k} .
$$


Using the symmetry of the operator $\pi_{k, \ell}$ we deduce

$$
2 \int_{\Gamma^{k, \ell}} \eta_{\ell, k} \psi_{\ell, k}=\int_{\Gamma^{k, \ell}}\left(\psi_{\ell, k}-\pi_{k, \ell} \psi_{\ell, k}\right) \eta_{\ell, k}+\frac{1}{\alpha} \int_{\Gamma^{k, \ell}}\left(e_{2}-\pi_{k, \ell}\left(e_{1}\right)\right) \psi_{\ell, k} .
$$

then, from (2.16) yields

$$
\left|\int_{\Gamma^{k, \ell}} \eta_{\ell, k} \psi_{\ell, k}\right| \leq c \sqrt{h}\left\|\eta_{\ell, k}\right\|_{L^{2}\left(\Gamma^{k, \ell}\right)}\left\|\psi_{\ell, k}\right\|_{H^{\frac{1}{2}}\left(\Gamma^{k, \ell}\right)}+\frac{1}{\alpha}\left\|e_{2}-\pi_{k, \ell}\left(e_{1}\right)\right\|_{H^{-\frac{1}{2}}\left(\Gamma^{k, \ell}\right)}\left\|\psi_{\ell, k}\right\|_{H^{\frac{1}{2}}\left(\Gamma^{k, \ell}\right)}
$$

and thus, we have

$$
\left\|\eta_{\ell, k}\right\|_{H^{-\frac{1}{2}}\left(\Gamma^{k, \ell}\right)} \leq c \sqrt{h}\left\|\eta_{\ell, k}\right\|_{L^{2}\left(\Gamma^{k, \ell}\right)}+\frac{c}{\alpha}\left\|e_{2}-\pi_{k, \ell}\left(e_{1}\right)\right\|_{H^{-\frac{1}{2}\left(\Gamma^{k, \ell}\right)}}
$$

Then, using (3.27) and the fact that

$$
\begin{array}{r}
\left\|e_{2}-\pi_{k, \ell}\left(e_{1}\right)\right\|_{H^{-\frac{1}{2}}\left(\Gamma^{k, \ell}\right)} \leq\left\|e_{2}\right\|_{H^{-\frac{1}{2}}\left(\Gamma^{k, \ell}\right)}+\left\|e_{1}\right\|_{H^{-\frac{1}{2}}\left(\Gamma^{k, \ell}\right)}+\left\|e_{1}-\pi_{k, \ell}\left(e_{1}\right)\right\|_{H^{-\frac{1}{2}}\left(\Gamma^{k, \ell}\right)} \\
\leq\left\|e_{2}\right\|_{H^{-\frac{1}{2}}\left(\Gamma^{k, \ell}\right)}+\left\|e_{1}\right\|_{H^{-\frac{1}{2}}\left(\Gamma^{k, \ell}\right)}+c \sqrt{h}\left\|e_{1}\right\|_{L^{2}\left(\Gamma^{k, \ell}\right)}
\end{array}
$$

with (3.26) and (3.30) yields for $0 \leq m \leq M-1$

$$
\left\|\eta_{\ell, k}\right\|_{H^{-\frac{1}{2}\left(\Gamma^{k, \ell}\right)}} \leq c h^{2+m}\left(\left\|u_{k}\right\|_{H^{2+m}\left(\Omega^{k}\right)}+\left\|u_{\ell}\right\|_{H^{2+m}\left(\Omega^{\ell}\right)}\right)+\frac{c h^{1+m}}{\alpha}\left\|p_{k, \ell}\right\|_{H^{\frac{1}{2}+m}\left(\Gamma^{k, \ell}\right)} .
$$

Using the previous inequality in (3.29), (3.28) yields

$$
\left\|\tilde{p}_{k \ell h}-p_{k, \ell}\right\|_{H^{-\frac{1}{2}\left(\Gamma^{k, \ell}\right)}} \leq c \alpha h^{2+m}\left(\left\|u_{k}\right\|_{H^{2+m}\left(\Omega^{k}\right)}+\left\|u_{\ell}\right\|_{H^{2+m}\left(\Omega^{\ell}\right)}\right)+c h^{1+m}\left\|p_{k, \ell}\right\|_{H^{\frac{1}{2}+m}\left(\Gamma^{k, \ell}\right)} .
$$

Let us now estimate $\left\|\tilde{u}_{\ell h}-u_{\ell}\right\|_{H^{1}\left(\Omega^{\ell}\right)}$ :

$$
\left\|\tilde{u}_{\ell h}-u_{\ell}\right\|_{H^{1}\left(\Omega^{\ell}\right)} \leq\left\|u_{\ell h}^{1}-u_{\ell}\right\|_{H^{1}\left(\Omega^{\ell}\right)}+\sum_{k<\ell}\left\|\mathcal{R}_{\ell, k}\left(\eta_{\ell, k}\right)\right\|_{H^{1}\left(\Omega^{\ell}\right)}
$$

and from (3.16)

$$
\left\|\mathcal{R}_{\ell, k}\left(\eta_{\ell, k}\right)\right\|_{H^{1}\left(\Omega^{\ell}\right)} \leq c\left\|\eta_{\ell, k}\right\|_{H_{00}^{\frac{1}{2}}\left(\Gamma^{k, \ell}\right)}
$$

then, with an inverse inequality

$$
\left\|\mathcal{R}_{\ell, k}\left(\eta_{\ell, k}\right)\right\|_{H^{1}\left(\Omega^{\ell}\right)} \leq c h^{-\frac{1}{2}}\left\|\eta_{\ell, k}\right\|_{L^{2}\left(\Gamma^{k, \ell}\right)} .
$$

Hence, from (3.27) we have for $0 \leq m \leq M-1$

$$
\begin{array}{r}
\left\|\mathcal{R}_{\ell, k}\left(\eta_{\ell, k}\right)\right\|_{H^{1}\left(\Omega^{\ell}\right)} \leq c h^{1+m}\left(\left\|u_{k}\right\|_{H^{2+m}\left(\Omega^{k}\right)}+\left\|u_{\ell}\right\|_{H^{2+m}\left(\Omega^{\ell}\right)}\right) \\
+\frac{c h^{m}}{\alpha}\left\|p_{k, \ell}\right\|_{H^{\frac{1}{2}+m}\left(\Gamma^{k, \ell}\right)}
\end{array}
$$

and (3.32) yields

$$
\begin{array}{r}
\left\|\tilde{u}_{\ell h}-u_{\ell}\right\|_{H^{1}\left(\Omega^{\ell}\right)} \leq c h^{1+m}\left\|u_{\ell}\right\|_{H^{2+m}\left(\Omega^{\ell}\right)}+c h^{1+m} \sum_{k<\ell}\left\|u_{k}\right\|_{H^{2+m}\left(\Omega^{k}\right)} \\
+\frac{c h^{m}}{\alpha} \sum_{k<\ell}\left\|p_{k, \ell}\right\|_{H^{\frac{1}{2}+m}\left(\Gamma^{k, \ell}\right)} .
\end{array}
$$


As $u \in H^{2+m}\left(\Omega^{\ell}\right)$,

$$
\left\|p_{\ell}\right\|_{H^{\frac{1}{2}+m}\left(\partial \Omega^{\ell}\right)} \leq c\left\|u_{\ell}\right\|_{H^{2+m}\left(\Omega^{\ell}\right)}
$$

and for $0 \leq m \leq M-1$

$$
\left\|\underline{\tilde{u}}_{h}-\underline{u}\right\|_{*} \leq \frac{c h^{m}}{\alpha} \sum_{i=1}^{K}\|\underline{u}\|_{H^{2+m}\left(\Omega^{k}\right)} .
$$

Proof of theorem 5: The proof is the same that for theorem 4, except that the relation (3.11) for $0 \leq m \leq M-1$ is changed using the following lemma

Lemma 5. $p_{k \ell h}^{1}$ satisfy for $0 \leq m \leq M-1$ the error estimate

$$
\left\|p_{k \ell h}^{1}-p_{k, \ell}\right\|_{L^{2}\left(\Gamma^{k, \ell}\right)} \leq c h^{\frac{3}{2}+m}(\log h)^{\beta(m)}\left\|p_{k, \ell}\right\|_{H^{\frac{3}{2}+m}\left(\Gamma^{k, \ell}\right)} .
$$

Therefore, (3.12) is changed in

$$
\left\|p_{k \ell h}^{1}-p_{k, \ell}\right\|_{H^{-\frac{1}{2}}\left(\Gamma^{k, \ell}\right)} \leq c h^{2+m}(\log h)^{\beta(m)}\left\|p_{k, \ell}\right\|_{H^{\frac{3}{2}+m}\left(\Gamma^{k, \ell}\right)}
$$

and (3.31) is changed in

$$
\begin{aligned}
& \left\|\tilde{p}_{k \ell h}-p_{k, \ell}\right\|_{H^{-\frac{1}{2}}\left(\Gamma^{k, \ell}\right)} \leq c \alpha h^{2+m}\left(\left\|u_{k}\right\|_{H^{2+m}\left(\Omega^{k}\right)}+\left\|u_{\ell}\right\|_{H^{2+m}\left(\Omega^{\ell}\right)}\right) \\
& +c h^{2+m}(\log h)^{\beta(m)}\left\|p_{k, \ell}\right\|_{H^{\frac{3}{2}+m}\left(\Gamma^{k, \ell}\right)}
\end{aligned}
$$

and (3.33) is changed in

$$
\begin{array}{r}
\left\|\tilde{u}_{\ell h}-u_{\ell}\right\|_{H^{1}\left(\Omega^{\ell}\right)} \leq c h^{1+m}\left\|u_{\ell}\right\|_{H^{2+m}\left(\Omega^{\ell}\right)}+c h^{1+m} \sum_{k<\ell}\left\|u_{k}\right\|_{H^{2+m}\left(\Omega^{k}\right)} \\
+\frac{c h^{1+m}}{\alpha}(\log h)^{\beta(m)} \sum_{k<\ell}\left\|p_{k, \ell}\right\|_{H^{\frac{3}{2}+m}\left(\Gamma^{k, \ell}\right)} .
\end{array}
$$

Proof of lemma 5: For $0 \leq m<M-1, \beta(m)=0$ and the estimate (3.34) is standard. For $m=M-1$, let $\bar{p}_{k \ell h}$ be the unique element of $\tilde{W}_{h}^{k, \ell}$ defined as follows :

- $\left(\bar{p}_{k \ell h}\right)_{\mid\left[x_{1}^{\ell, k}, x_{n-1}^{\ell, k}\right]}$ coincide with the interpolate of degree $M$ of $p_{k, \ell}$.

- $\left(\bar{p}_{k \ell h}\right)_{\mid\left[x_{0}^{\ell, k}, x_{1}^{\ell, k}\right]}$ and $\left(\bar{p}_{k \ell h}\right)_{\mid\left[x_{n-1}^{\ell, k}, x_{n}^{\ell, k}\right]}$ coincide with the interpolate of degree $M-1$ of $p_{k, \ell}$.

Then, we have

$$
\left\|p_{k \ell h}^{1}-p_{k, \ell}\right\|_{L^{2}\left(\Gamma^{k, \ell}\right)}^{2} \leq\left\|\bar{p}_{k \ell h}-p_{k, \ell}\right\|_{L^{2}\left(\Gamma^{k, \ell}\right)}^{2} .
$$

Using Deny-Lions theorem we have

$\left\|p_{k \ell h}^{1}-p_{k, \ell}\right\|_{L^{2}\left(\Gamma^{k, \ell}\right)}^{2} \leq h^{2 M} \int_{x_{0}^{\ell, k}}^{x_{1}^{\ell, k}}\left|\frac{d^{M} p_{k, \ell}}{d x^{M}}\right|^{2}+h^{2\left(M+\frac{1}{2}\right)}\left\|p_{k, \ell}\right\|_{H^{M+\frac{1}{2}\left(\left[x_{1}^{\ell, k}, x_{n-1}^{\ell, k}\right]\right)}}+h^{2 M} \int_{x_{n-1}^{\ell, k}}^{x_{n}^{\ell, k}}\left|\frac{d^{M} p_{k, \ell}}{d x^{M}}\right|^{2}$.

Let $\varphi=\frac{d^{M} p_{k, \ell}}{d x^{M}}$. In order to analyse the two extreme contributions, we use Hölder's inequality :

$$
\int_{0}^{h} \varphi^{2} \leq h^{1-\frac{1}{p}}\|\varphi\|_{L^{p}(0, h)}^{2}
$$


Then, we use the estimate

$$
\|\varphi\|_{L^{p}(0, h)} \leq c p\|\varphi\|_{H^{\frac{1}{2}(0, h)}}
$$

where $c$ is a constant. Thus we have

$$
\int_{0}^{h} \varphi^{2} \leq c p^{2} h^{1-\frac{1}{p}}\|\varphi\|_{H^{\frac{1}{2}}(0, h)}^{2} .
$$

Then,

$$
h^{2 M} \int_{x_{0}^{\ell, k}}^{x_{1}^{\ell, k}}\left|\frac{d^{M} p_{k, \ell}}{d x^{M}}\right|^{2} \leq c h^{2 M} p^{2} h^{1-\frac{1}{p}}\left\|\frac{d^{M} p_{k, \ell}}{d x^{M}}\right\|_{H^{\frac{1}{2}}}^{2}\left(x_{0}^{\ell, k}, x_{1}^{\ell, k}\right) .
$$

Now we take $p=\log h$ and thus we obtain

$$
h^{2 M} \int_{x_{0}^{\ell, k}}^{x_{1}^{\ell, k}}\left|\frac{d^{M} p_{k, \ell}}{d x^{M}}\right|^{2} \leq c\left(h^{M+\frac{1}{2}} \log h\right)^{2}\left\|p_{k, \ell}\right\|_{H^{\frac{1}{2}+M}\left(x_{0}^{\ell, k}, x_{1}^{\ell, k}\right)}^{2} .
$$

In a same way we have

$$
h^{2 M} \int_{x_{n-1}^{\ell, k}}^{x_{n}^{\ell, k}}\left|\frac{d^{M} p_{k, \ell}}{d x^{M}}\right|^{2} \leq c\left(h^{M+\frac{1}{2}} \log h\right)^{2}\left\|p_{k, \ell}\right\|_{H^{\frac{1}{2}+M}\left(x_{n-1}^{\ell, k}, x_{n}^{\ell, k}\right)}^{2},
$$

and thus we obtain

$$
\left\|p_{k \ell h}^{1}-p_{k, \ell}\right\|_{L^{2}\left(\Gamma^{k, \ell}\right)} \leq c\left(h^{M+\frac{1}{2}} \log h\right)\left\|p_{k, \ell}\right\|_{H^{\frac{1}{2}+M}\left(\Gamma^{k, \ell}\right)},
$$

which ends the proof of lemma 5 .

3.3. Error Estimates.. Thanks to (3.5), we have the following error estimates:

THEOREM 6. Assume that the solution $u$ of (2.1)-(2.2) is in $H^{2}(\Omega) \cap H_{0}^{1}(\Omega)$, and $u_{k}=u_{\mid \Omega^{k}} \in H^{2+m}\left(\Omega^{k}\right)$, with $M-1 \geq m \geq 0$, and let $p_{k, \ell}=\frac{\partial u}{\partial \mathbf{n}_{k}}$ over each $\Gamma^{k, \ell}$. Then, there exists a constant $c$ independent of $h$ and $\alpha$ such that

$$
\begin{array}{r}
\left\|\underline{u}_{h}-\underline{u}\right\|_{*}+\left\|\underline{p}_{h}-\underline{p}\right\|_{-\frac{1}{2}, *} \leq c\left(\alpha h^{2+m}+h^{1+m}\right) \sum_{k=1}^{K}\|\underline{u}\|_{H^{2+m}\left(\Omega^{k}\right)} \\
+c\left(\frac{h^{m}}{\alpha}+h^{1+m}\right) \sum_{k=1}^{K} \sum_{\ell}\left\|p_{k, \ell}\right\|_{H^{\frac{1}{2}+m}\left(\Gamma^{k, \ell}\right)} .
\end{array}
$$

Theorem 7. Assume that the solution $u$ of (2.1)-(2.2) is in $H^{2}(\Omega) \cap H_{0}^{1}(\Omega)$, $u_{k}=u_{\mid \Omega^{k}} \in H^{2+m}\left(\Omega^{k}\right)$, and $p_{k, \ell}=\frac{\partial u}{\partial \mathbf{n}_{k}}$ is in $H^{\frac{3}{2}+m}\left(\Gamma_{k, \ell}\right)$ with $M-1 \geq m \geq 0$. Then there exists a constant $c$ independent of $h$ and $\alpha$ such that

$$
\begin{aligned}
\left\|\underline{u}_{h}-\underline{u}\right\|_{*} & +\left\|\underline{p}_{h}-\underline{p}\right\|_{-\frac{1}{2}, *} \leq c\left(\alpha h^{2+m}+h^{1+m}\right) \sum_{k=1}^{K} \| \underline{u}_{H^{2+m}\left(\Omega^{k}\right)} \\
& +c\left(\frac{h^{1+m}}{\alpha}+h^{2+m}\right)(\log h)^{\beta(m)} \sum_{k=1}^{K} \sum_{\ell}\left\|p_{k, \ell}\right\|_{H^{\frac{3}{2}+m}\left(\Gamma^{k, \ell}\right)}
\end{aligned}
$$


with $\beta(m)=0$ if $m \leq M-2$ and $\beta(m)=1$ if $m=M-1$.

REMARK 1. Let us consider a $P_{1}$ finite element approximation. If the solution $\underline{u}$ of (2.1)-(2.2) is in $\prod_{k=1}^{K} H_{*}^{2}\left(\Omega^{k}\right)$ and $\alpha$ is a constant independent of $h$ then, from theorem [6.

$$
\left\|\underline{\tilde{u}}_{h}-\underline{u}\right\|_{*} \leq c \sum_{i=1}^{K}\|\underline{u}\|_{H^{2}\left(\Omega^{k}\right)}
$$

and this result is not optimal. In order to improve this fact, we have to choose a parameter $\alpha$ which depends on $h$, or assume that $\underline{u} \in \prod_{k=1}^{K} H_{*}^{3}\left(\Omega^{k}\right)$, or assume that $\underline{u} \in \prod_{k=1}^{K} H_{*}^{2}\left(\Omega^{k}\right)$ and $p_{k, \ell}=\frac{\partial u}{\partial \mathbf{n}_{k}} \in H^{\frac{3}{2}}\left(\Gamma_{k, \ell}\right)$ :

- If the solution $\underline{u}$ of (2.1)-(2.2) is in $\prod_{k=1}^{K} H_{*}^{2}\left(\Omega^{k}\right)$ and $\alpha=\frac{c}{h}$, then

$$
\left\|\underline{\tilde{u}}_{h}-\underline{u}\right\|_{*} \leq \operatorname{ch} \sum_{i=1}^{K}\|\underline{u}\|_{H^{2}\left(\Omega^{k}\right)}
$$

- If the solution $\underline{u}$ of (2.1)-(2.2) is in $\prod_{k=1}^{K} H_{*}^{2}\left(\Omega^{k}\right), p_{k, \ell}=\frac{\partial u}{\partial \mathbf{n}_{k}}$ is in $H^{\frac{3}{2}}\left(\Gamma_{k, \ell}\right)$ and $\alpha$ is a constant independent of $h$ then

$$
\left\|\underline{\tilde{u}}_{h}-\underline{u}\right\|_{*}=O(h|\log (h)|) .
$$

3.4. Analysis of the best fit in $3 \mathrm{D}$. In this section, we prove lemma 4 for a $P_{1}$-discretization in $3 \mathrm{D}$. We shall use the construction proposed in [11. In order to make the reading easy, we shall recall the notations of the above mentioned paper. The analysis is done on one subdomain $\Omega^{k}$ that will be fixed in what follows. A typical interface between this subdomain and a generic subdomain $\Omega_{l}$ will be denoted by $\Gamma$. We denote by $\mathcal{T}$ the restriction to $\Gamma$ of the triangulation $\mathcal{T}_{h}^{k}$. Let $S(\mathcal{T})$ denote the space of piecewise linear functions with respect to $\mathcal{T}$ which are continuous on $\Gamma$ and vanish on its boundary. The space of the Lagrange multipliers on $\Gamma$, defined below, will be denoted by $M(\mathcal{T})$. In $2 \mathrm{D}$, the requirement $\operatorname{dim} M(\mathcal{T})=\operatorname{dim} S(\mathcal{T})$ can be satisfied by lowering the degree of the finite elements on the intervals next to the end points of the interface. In 3D, it is slightly more complex (see [5]). Thus, we shall use the construction proposed in [11] in the case where all the vertices of the boundary of $\Gamma$ are connected to zero or two vertices in the interior of $\Gamma$ (figure 3.1). Let $\mathcal{V}, \mathcal{V}_{0}$, $\partial \mathcal{V}$ denote respectively the set of all the vertices of $\mathcal{T}$, the vertices in the interior of $\Gamma$, and the vertices on the boundary of $\Gamma$. The finite element basis functions will be denoted by $\Phi_{a}, a \in \mathcal{V}$. Thus,

$$
S(\mathcal{T})=\operatorname{span}\left\{\Phi_{a}: a \in \mathcal{V}_{0}\right\}
$$

For $a \in \mathcal{V}$, let $\sigma_{a}$ denote the support of $\Phi_{a}$,

$$
\sigma_{a}:=\bigcup\{T \in \mathcal{T}: a \in T\},
$$

and let $\mathcal{N}_{a}$ be the set of neighboring vertices in $\mathcal{V}_{0}$ of a:

$$
\mathcal{N}_{a}:=\left\{b \in \mathcal{V}_{0}: b \in \sigma_{a}\right\} .
$$

Thus,

$$
\mathcal{N}=\bigcup_{a \in \partial \mathcal{V}} \mathcal{N}_{a}
$$


is the set of those interior vertices which have a neighbor on the boundary of $\Gamma$. If some triangle $T \in \mathcal{T}$ has all its vertices on the boundary of $\Gamma$, then there exists one (corner) vertex which has no neighbor in $\mathcal{V}_{0}$. Let $\mathcal{T}_{c}$ be the set of triangles $T \in \mathcal{T}$ which have all their vertices on the boundary of $\Gamma$. For $T \in \mathcal{T}_{c}$, we denote by $c_{T}$ the only vertex of $T$ that has no interior neighbour (such a vertex is unique as soon as the triangulation is fine enough). Let $\mathcal{N}_{c}$ denote the vertices $a_{T}$ of $\mathcal{N}$ which belong to a triangle adjacent to a triangle $T \in \mathcal{T}_{c}$. Now, we define the space $M(\mathcal{T})$ by

$$
M(\mathcal{T}):=\operatorname{span}\left\{\hat{\Phi}_{a}, a \in \mathcal{V}_{0}\right\}
$$

where the basis functions $\hat{\Phi}_{a}$ are defined as follows :

$$
\hat{\Phi}_{a}:= \begin{cases}\Phi_{a}, & a \in \mathcal{V}_{0} \backslash \mathcal{N} \\ \Phi_{a}+\sum_{b \in \partial \mathcal{V} \cap \sigma_{a}} A_{b, a} \Phi_{b} & a \in \mathcal{N} \backslash \mathcal{N}_{c} \\ \Phi_{a_{T}}+\sum_{b \in \partial \mathcal{V} \cap \sigma_{a_{T}}} A_{b, a_{T}} \Phi_{b}+\Phi_{c_{T}} & a=a_{T} \in \mathcal{N}_{c}\end{cases}
$$

the weights $A_{b, a}$ being defined in (3.35). For all boundary nodes $c \in \partial \mathcal{V}$ connected to two interior nodes $a$ and $b$, if $T_{a}$ (resp. $T_{b}$ ) denote the triangle having an edge on $\partial \Gamma$ and $a$ (resp. $b$ ) as the opposite vertex, then the weights are defined such that (see [11)

$$
A_{c, a}+A_{c, b}=1 \text { and }\left|T_{b}\right| A_{c, a}=\left|T_{a}\right| A_{c, b} .
$$

$M(\mathcal{T})$ is the notation introduced in [11, that we use here for the sake of clarity. Corresponding to our previous notation, $M(\mathcal{T}) \equiv \tilde{W}_{h}^{k, \ell}$.

To any $u \in S(\mathcal{T}), u=\sum_{a \in \mathcal{V}_{0}} u(a) \Phi_{a}$, we associate $v \in M(\mathcal{T})$ where $v=\sum_{a \in \mathcal{V}_{0}} u(a) \hat{\Phi}_{a}$. More explicitly, that means that to any $u \in S(\mathcal{T})$, we associate an element $v \in M(\mathcal{T})$ as follows (see figure 3.1):

(i) $v$ is a piecewise linear finite element on $\mathcal{T}$

(ii) for all interior nodes $a, v(a):=u(a)$

(iii) for all boundary nodes $c$, by assumption we have two situations:

- $c$ is connected to two interior nodes denoted by $a$ and $b$.

Then, $v(c):=A u(a)+B u(b)$ where

$$
A+B=1 \text { and }\left|T_{b}\right| A=\left|T_{a}\right| B
$$

where $T_{a}$ (resp. $T_{b}$ ) is the triangle having an edge on $\partial \Gamma$ and $a$ (resp. $b$ ) as the opposite vertex.

- $c$ is not connected to any interior point. We consider the triangle adjacent to the triangle to which $c$ belongs to. This triangle has one interior node denoted by $b$. Then, we define $v(b):=u(b)$.

Then, using the uniform regularity of $\mathcal{T}$, it is easy to check that there exists a constant $c$ independent of $h$ such that

$$
\|v\|_{L^{2}(\Gamma)} \leq c\|u\|_{L^{2}(\Gamma)} .
$$

We shall need the following technical assumption:

Assumption Let $0<C \leq 2 / 3$. For any triangle $T_{c^{\prime}}$ having all three vertices on the 
boundary of $\mathcal{T}$ (see figure 3.1), we consider the two triangles $T_{i, c}$ and $T_{j^{\prime}, c}$ surrounding $T_{c^{\prime}}$. We assume that

$$
\frac{1}{24} \min \left(\left|T_{i, c}\right|,\left|T_{j^{\prime}, c}\right|\right)>\frac{C}{2}\left|T_{c^{\prime}}\right|
$$

In order to prove lemma 4, we prove the following lemma:

Lemma 6. Let $0<C \leq 2 / 3$, we assume the above assumption and that $\mathcal{T}$ is uniformly regular. Let $u \in S(\mathcal{T})$ and let $v \in M(\mathcal{T})$ constructed from $u$ as explained above ((i)-(iii)).

Then, there exists $c>0$ such that,

$$
\int_{\Gamma}\left(u v-\frac{C}{2}(u-v)^{2}\right) \geq c \int_{\Gamma} u^{2} .
$$

Proof of lemma 6: Let us introduce the notation

$$
Q_{\Gamma}:=\int_{\Gamma}\left(u v-\frac{C}{2}(u-v)^{2}\right) .
$$

We have

$$
Q_{\Gamma}=\frac{1}{4} \int_{\Gamma}(u+v)^{2}-(1+2 C)(u-v)^{2} .
$$

In order to estimate $Q_{\Gamma}$, we remark that

$$
Q_{\Gamma}=\sum_{T \in \mathcal{T}} Q_{T}
$$

where

$$
Q_{T}=\frac{1}{4} \int_{T}(u+v)^{2}-(1+2 C)(u-v)^{2} .
$$

We have four kinds of triangles:

1. Inner triangles i.e they don't touch the boundary of $\Gamma$.

2. Triangles which have only one vertex on the boundary

3. Triangles which have two vertices on the boundary

4. Triangles which have three vertices on the boundary

Inner triangles. On an inner triangle $T, u=v$ so that for all $C>0$, we have

$$
Q_{T} \geq c \int_{T} u^{2}
$$

for $c \leq 1$.

Triangles having only one vertex on the boundary. Let $T_{i, c}$ be such a triangle (see figure 3.1). We use the following notations: $u_{i}=u(a)=v(a), u_{i+1}=u(b)=v(b)$ and $v_{i}=v(c)$. First notice that we have (remember $u(c)=0$ )

$$
\int_{T_{i, c}} u^{2}=\frac{\left|T_{i, c}\right|}{12}\left(u_{i}^{2}+u_{i+1}^{2}+\left(u_{i}+u_{i+1}\right)^{2}\right)=\frac{\left|T_{i, c}\right|}{12}\left(2 u_{i}^{2}+2 u_{i+1}^{2}+2 u_{i} u_{i+1}\right)
$$


see for example [10] (II.8.4). As for $Q_{T_{i, c}}$, we have

$$
\begin{array}{r}
Q_{T_{i, c}}=\frac{\left|T_{i, c}\right|}{48}\left(\left(2 u_{i}\right)^{2}+\left(2 u_{i+1}\right)^{2}+\left(A u_{i}+B u_{i+1}\right)^{2}\right. \\
\left.+\left(2 u_{i}+2 u_{i+1}+A u_{i}+B u_{i+1}\right)^{2}-2(1+2 C)\left(A u_{i}+B u_{i+1}\right)^{2}\right) \\
=\frac{\left|T_{i, c}\right|}{48}\left(8 u_{i}^{2}+8 u_{i+1}^{2}+8 u_{i} u_{i+1}+4\left(u_{i}+u_{i+1}\right)\left(A u_{i}+B u_{i+1}\right)\right. \\
\left.-4 C\left(A u_{i}+B u_{i+1}\right)^{2} .\right)
\end{array}
$$

If we take $C=1$ and use $A+B=1$, we get:

$$
\begin{aligned}
Q_{T_{i, c}} & =\frac{\left|T_{i, c}\right|}{48}\left(4 u_{i}^{2}+4 u_{i+1}^{2}+4 u_{i} u_{i+1}+4 A B\left(u_{i}-u_{i+1}\right)^{2}+4\left(u_{i}+u_{i+1}\right)^{2}\right) \\
& \geq \frac{1}{2} \int_{T_{i, c}} u^{2} .
\end{aligned}
$$

Hence, for all $0<C \leq 1$, we have:

$$
Q_{T_{i, c}} \geq \frac{1}{2} \int_{T_{i, c}} u^{2}
$$

Therefore,

$$
Q_{T_{i, c}} \geq c \int_{T_{i, c}} u^{2}
$$

for $0<C \leq 1$ and $0<c \leq 1 / 2$. We shall also use in the sequel the estimate:

$$
Q_{T_{i, c}} \geq \frac{\left|T_{i, c}\right|}{24} u_{i+1}^{2} .
$$

Triangles having two vertices on the boundary. We consider now a triangle $T_{i, r}$ having two vertices on the boundary of the face $\Gamma$, see figure 3.1. Let $\mathcal{N}_{r}=\left\{i, T_{i, r}\right.$ has two vertices on the boundary of $\Gamma$ \}. First notice that we have

$$
\int_{T_{i, r}} u^{2}=\frac{\left|T_{i, c}\right|}{12}\left(2 u_{i+1}^{2}\right) \text {. }
$$

And we have

$$
\begin{aligned}
Q_{T_{i, r}}= & \frac{\left|T_{i, r}\right|}{48}\left(4 u_{i+1}^{2}+v_{i}^{2}+v_{i+1}^{2}+\left(2 u_{i+1}+v_{i}+v_{i+1}\right)^{2}\right. \\
& \left.-(1+2 C)\left(v_{i}^{2}+v_{i+1}^{2}+\left(v_{i}+v_{i+1}\right)^{2}\right)\right) \\
= & \frac{\left|T_{i, r}\right|}{48}\left(8 u_{i+1}^{2}-4 C v_{i}^{2}-4 C v_{i+1}^{2}-4 C v_{i} v_{i+1}+4 u_{i+1}\left(v_{i}+v_{i+1}\right)\right) .
\end{aligned}
$$

Then,

$$
Q_{T_{i, r}} \geq \frac{\left|T_{i, r}\right|}{48}\left(8 u_{i+1}^{2}-6 C v_{i}^{2}-6 C v_{i+1}^{2}+4 u_{i+1}\left(v_{i}+v_{i+1}\right)\right) .
$$


Defining $E_{i}:=u_{i+1} v_{i}$ and $F_{i}:=u_{i+1} v_{i+1}$ (cf. [11] page 11), we have:

$$
Q_{T_{i, r}} \geq \int_{T_{i, r}} u^{2}+\frac{\left|T_{i, r}\right|}{48}\left(-6 C v_{i}^{2}+4 E_{i}-6 C v_{i+1}^{2}+4 F_{i}\right) .
$$

Now we sum these terms over all the triangles having two vertices on the boundary $\Gamma$.

$$
\begin{aligned}
& \sum_{i \in \mathcal{N}_{r}} Q_{T_{i, r}} \geq \int_{\cup_{i \in \mathcal{N}_{r} T_{i, r}}} u^{2}+\sum_{i \in \mathcal{N}_{r}} \frac{\left|T_{i, r}\right|}{48}\left(-6 C v_{i}^{2}+4 E_{i}-6 C v_{i+1}^{2}+4 F_{i}\right) \\
& \geq \int_{\cup_{i \in \mathcal{N}_{r} T_{i, r}}} u^{2}+\frac{1}{48} \sum_{i \in \mathcal{N}_{r}}\left(\left|T_{i, r}\right|\left(-6 C v_{i}^{2}+4 E_{i}\right)+\left|T_{i-1, r}\right|\left(-6 C v_{i}^{2}+4 F_{i-1}\right)\right) .
\end{aligned}
$$

The condition (3.36) leads to the inequality

$$
\left|T_{i, r}\right| E_{i}+\left|T_{i-1, r}\right| F_{i-1}=\left(\left|T_{i, r}\right|+\left|T_{i-1, r}\right|\right) v_{i}^{2}
$$

(see equation after (3.19) in [1]), so that we get:

$$
\left|T_{i, r}\right|\left(-6 C v_{i}^{2}+4 E_{i}\right)+\left|T_{i-1, r}\right|\left(-6 C v_{i}^{2}+4 F_{i-1}\right)=\left(\left|T_{i, r}\right|+\left|T_{i-1, r}\right|\right)(4-6 C) v_{i}^{2} .
$$

This term cancels for $C=2 / 3$. Hence for $0<C \leq 2 / 3$, inequality (3.39) becomes:

$$
\sum_{i \in \mathcal{N}_{r}} Q_{T_{i, r}} \geq \int_{\cup_{i \in \mathcal{N}_{r} T_{i, r}}} u^{2}
$$

Therefore, for $0<C \leq 2 / 3$ and $0<c \leq 1$,

$$
\sum_{i \in \mathcal{N}_{r}} Q_{T_{i, r}} \geq c \int_{\cup_{i \in \mathcal{N}_{r}} T_{i, r}} u^{2}
$$

Triangles having all three vertices on the boundary. Let $T_{c^{\prime}}$ be such a triangle (see figure 3.1). We have to control:

$$
Q_{T_{c^{\prime}}}=-\frac{C}{2}\left|T_{c^{\prime}}\right|\left|u_{i+1}\right|^{2}
$$

by the integrals over the two triangles $T_{i, c}$ and $T_{j^{\prime}, c}$ surrounding $T_{c^{\prime}}$. This can be achieved using the assumption

$$
\frac{1}{24} \min \left(\left|T_{i, c}\right|,\left|T_{j^{\prime}, c}\right|\right)>\frac{C}{2}\left|T_{c^{\prime}}\right|
$$

and using that from (3.38), we have

$$
Q_{T_{i, c} \cup T_{j^{\prime}, c}} \geq \min \left(\left|T_{i, c}\right|,\left|T_{j^{\prime}, c}\right|\right) \frac{u_{i+1}^{2}}{12} .
$$

In conclusion, we have that (3.37) holds with $c=1 / 4$ for $0<C \leq 2 / 3$. 


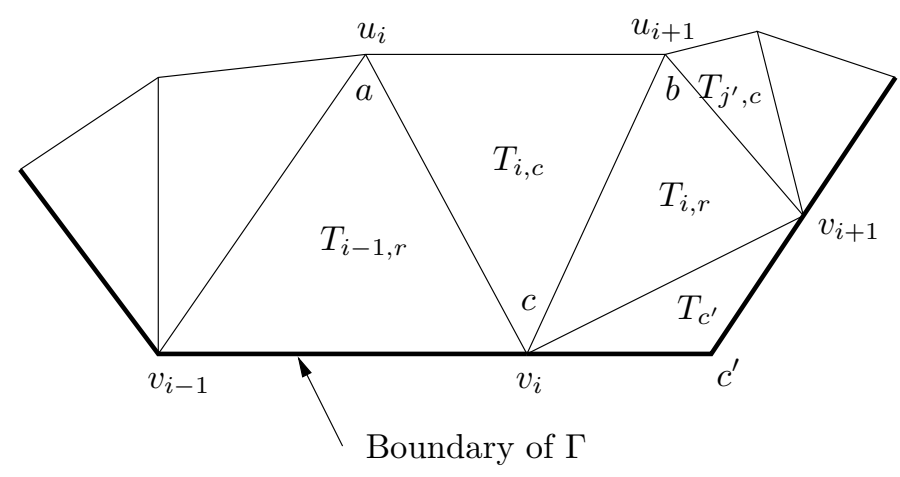

FIG. 3.1. Two different situations of $2 D$ triangulation of the interface $\Gamma$, next to it's boundary (near cross points)

4. Numerical results. On the unit square $\Omega=(0,1) \times(0,1)$ we consider the problem

$$
\begin{aligned}
(I d-\Delta) u(x, y) & =x^{3}\left(y^{2}-2\right)-6 x y^{2}+\left(1+x^{2}+y^{2}\right) \sin (x y), \quad(x, y) \in \Omega, \\
u & =x^{3} y^{2}+\sin (x y), \quad(x, y) \in \partial \Omega
\end{aligned}
$$

whose exact solution is $u(x, y)=x^{3} y^{2}+\sin (x y)$. We decompose the unit square into non-overlapping subdomains with meshes generated in an independent manner. The computed solution is the solution at convergence of the discrete algorithm (2.11)(2.12), with a stopping criterion on the jumps of interface conditions that must be smaller than $10^{-8}$.

REMARK 2. In the implementation of the method, the main difficulty lies in computing projections between non matching grids. In [18] we present an efficient algorithm in two dimensions to perform the required projections between arbitrary grids, in the same spirit as in [19] for finite volume discretization with projections on piecewise constant functions.

4.1. Choice of the Robin parameter $\alpha$. In our simulations the Robin parameter is either an arbitrary constant or is obtained by minimizing the convergence rate (and depend of the mesh size in that case). In the conforming two subdomains case, with constant mesh size $h$, the optimal theoretical value of $\alpha$ which minimizes the convergence rate at the continuous level is :

$$
\alpha_{\text {opt }}=\left[\left(\pi^{2}+1\right)\left(\left(\frac{\pi}{h}\right)^{2}+1\right)\right]^{\frac{1}{4}} .
$$

In the non-conforming case, has the mesh size is different for each side of the interface, we consider the following values :

$$
\begin{aligned}
\alpha_{\text {min }} & =\left[\left(\pi^{2}+1\right)\left(\left(\frac{\pi}{h_{\text {min }}}\right)^{2}+1\right)\right]^{\frac{1}{4}} \\
\alpha_{\text {mean }} & =\left[\left(\pi^{2}+1\right)\left(\left(\frac{\pi}{h_{\text {mean }}}\right)^{2}+1\right)\right]^{\frac{1}{4}} \\
\alpha_{\text {max }} & =\left[\left(\pi^{2}+1\right)\left(\left(\frac{\pi}{h_{\text {max }}}\right)^{2}+1\right)\right]^{\frac{1}{4}}
\end{aligned}
$$

where $h_{\text {min }}, h_{\text {mean }}$ and $h_{\max }$ stands respectively for the smallest meanest or highest step size on the interface. 
4.2. An example of computed solution. We decompose the unit square into four non-overlapping subdomains with meshes generated as shown in Figure 4.1. The Robin parameter is $\alpha=10$. On Figure 4.2 we show that the computed solution is close to the continuous solution.

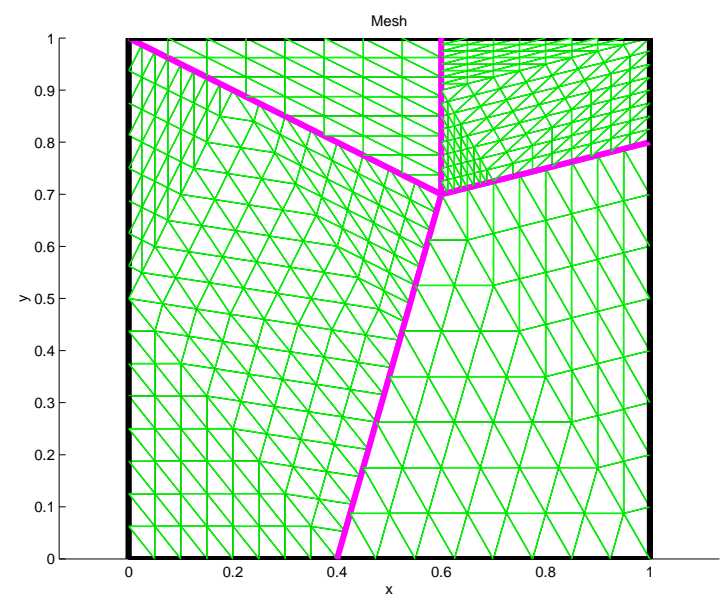

FIG. 4.1. Domain decomposition with non-conforming meshes.

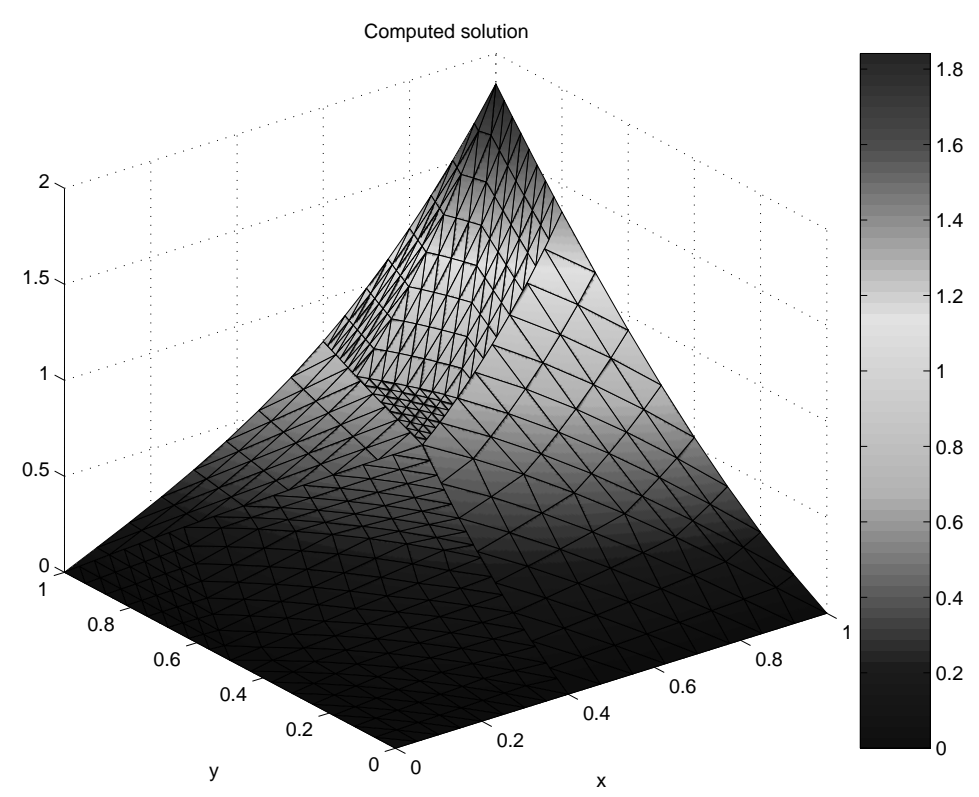

FIG. 4.2. Computed solution.

4.3. $H^{1}$ error between the continuous and discrete solutions. In this part, we compare the relative $H^{1}$ error in the non-conforming case to the error obtained on a uniform conforming grid.

Definition of the relative $H^{1}$ error : Let $K$ be the number of subdomains. Let 
$u_{i}=u_{\mid \Omega^{i}}, 1 \leq i \leq K$ (where $\mathrm{u}$ is the continuous solution), and let $\left(\underline{u}_{h}\right)_{i}=\left(\underline{u}_{h}\right)_{\mid \Omega^{i}}$ where $\underline{u}_{h}$ is the solution of the discrete problem (2.10). Now, let $E_{e x}=\|u\|_{*}$ and let $E_{i}=\left\|\left(\underline{u}_{h}\right)_{i}-u_{i}\right\|_{H^{1}\left(\Omega^{i}\right)}, 1 \leq i \leq K$. Let

$$
E=\left(\sum_{i=1}^{K} E_{i}^{2}\right)^{1 / 2} .
$$

The relative $H^{1}$ error is then $E / E_{e x}$.

In this example, we take $\alpha=\alpha_{\text {mean }}$ for the Robin parameter. This choice is motivated by the results of section 4.4, but we obtain similar results in the case of $\alpha=10$.

We consider four initial meshes : the two uniform conforming meshes (mesh 1 and 4) of figure 4.3, and the two non-conforming meshes (mesh 2 and 3) of figure 4.4. In the non-conforming case, the unit square is decomposed into four non-overlapping subdomains numbered as in figure 4.5
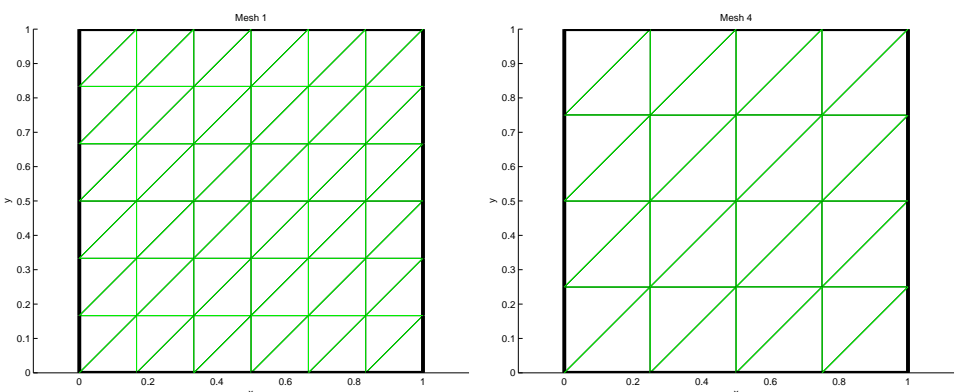

FIG. 4.3. Uniform conforming meshes : mesh 1 (on the left), and mesh 4 (on the right)
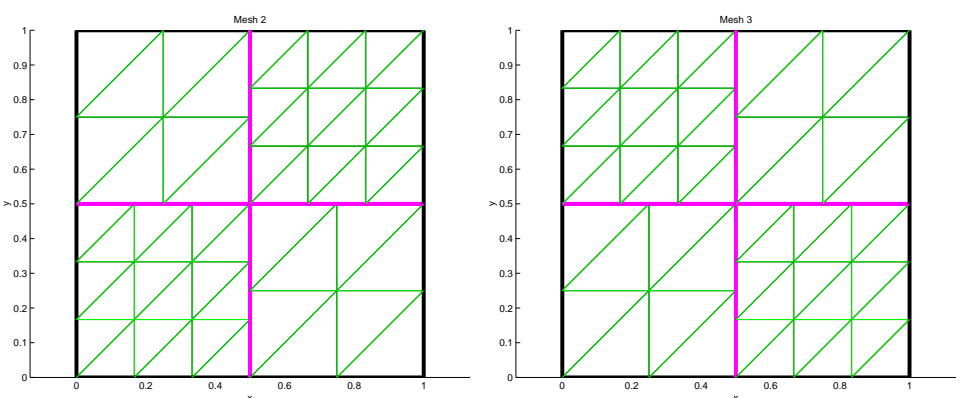

FIG. 4.4. Non-conforming meshes : mesh 2 (on the left), and mesh 3 (on the right)

\begin{tabular}{|l|l|}
\hline$\Omega^{3}$ & $\Omega^{4}$ \\
\hline$\Omega^{1}$ & $\Omega^{2}$ \\
\hline
\end{tabular}

FIG. 4.5. Non-overlapping domain decomposition of the unit square 
Figure 4.6 shows the relative $H^{1}$ error versus the number of refinement for these four meshes, and the mesh size $h$ versus the number of refinement, in logarithmic scale. At each refinement, the mesh size is divided by two. The results of figure 4.6 show that the relative $H^{1}$ error tends to zero at the same rate than the mesh size, and this fits with the theoretical error estimates of theorem 4, On the other hand, we observe that the two curves corresponding to the non-conforming meshes (mesh 2 and mesh 3) are between the curves of the conforming meshes (mesh 1 and mesh 4). The relative $H^{1}$ error for mesh 2 is smaller than the one corresponding to mesh 3, and this is because mesh 2 is more refined than mesh 3 in subdomain $\Omega^{4}$, where the solution steeply varies.

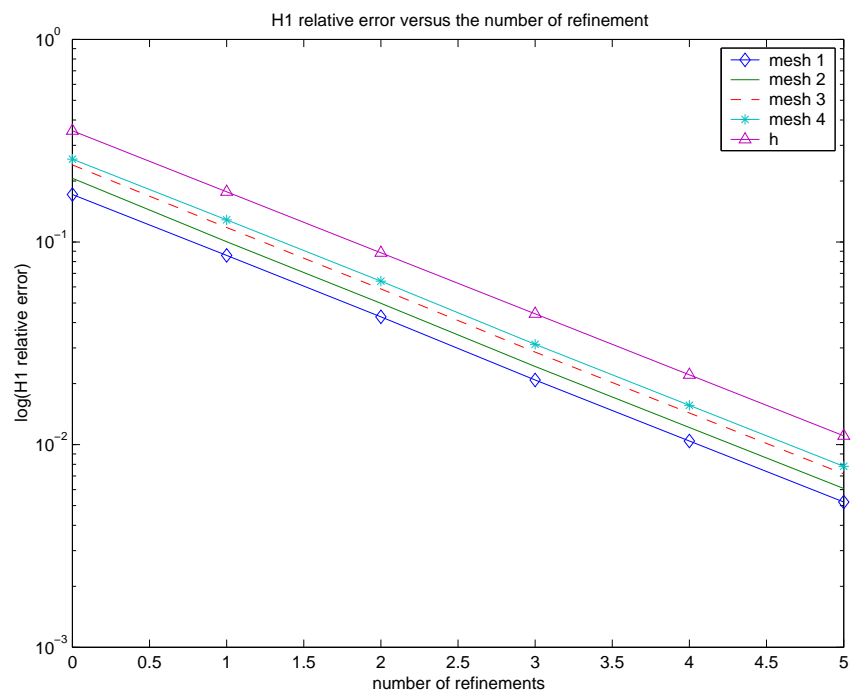

FIG. 4.6. relative $H^{1}$ error versus the number of refinements for the initial meshes : mesh 1 , (diamond line), mesh 2 (solid line), mesh 3 (dashed line), and mesh 4 (star line). The triangle line is the mesh size $h$ versus the number of refinements, in logarithmic scale

More precisely, let us compare for mesh 2, the relative $H^{1}$ error in the domain $\Omega^{1} \cup \Omega^{2} \cup \Omega^{3}$ to the relative $H^{1}$ error in the subdomain $\Omega^{4}$ (which is the subdomain where the solution steeply varies). This comparison can be done in Table 1.

\begin{tabular}{|c|c|c|c|}
\hline Refinement & $\left(E_{1}^{2}+E_{2}^{2}+E_{3}^{2}\right)^{1 / 2} / E_{e x}$ & $E_{4} / E_{e x}$ & $E / E_{e x}$ \\
\hline 0 & $1.45 \mathrm{e}-01$ & $1.46 \mathrm{e}-01$ & $2.06 \mathrm{e}-01$ \\
\hline 1 & $7.17 \mathrm{e}-02$ & $7.02 \mathrm{e}-02$ & $1.004 \mathrm{e}-01$ \\
\hline 2 & $3.59 \mathrm{e}-02$ & $3.49 \mathrm{e}-02$ & $5.01 \mathrm{e}-02$ \\
\hline 3 & $1.79 \mathrm{e}-02$ & $1.73 \mathrm{e}-02$ & $2.49 \mathrm{e}-02$ \\
\hline 4 & $8.73 \mathrm{e}-03$ & $8.46 \mathrm{e}-03$ & $1.21 \mathrm{e}-02$ \\
\hline
\end{tabular}

Table 1: Comparison, in the case of mesh 2, for different refinements (column one), of the relative $H^{1}$ error in the domain composed by subdomains $\Omega^{1}, \Omega^{2}$ and $\Omega^{3}$

(column 2) to the relative $H^{1}$ error in the subdomain $\Omega^{4}$ (column 3). The fourth column is the relative $H^{1}$ error in the whole domain.

We observe that, as expected, the relative $H^{1}$ error in the domain composed by subdomains $\Omega^{1}, \Omega^{2}$ and $\Omega^{3}$ (second column of table 1 ) is close to the relative $H^{1}$ error in the subdomain $\Omega^{4}$ (third column of table 1 ). Indeed, the mesh 2 is more refined in the subdomain $\Omega^{4}$ where the solution steeply varies. 


\begin{tabular}{|c|c|c|c|}
\hline Refinement & $\left(E_{1}^{2}+E_{2}^{2}+E_{3}^{2}\right)^{1 / 2} / E_{e x}$ & $E_{4} / E_{e x}$ & $E / E_{e x}$ \\
\hline 0 & $1.26 \mathrm{e}-01$ & $2.04 \mathrm{e}-01$ & $2.40 \mathrm{e}-01$ \\
\hline 1 & $5.57 \mathrm{e}-02$ & $1.04 \mathrm{e}-01$ & $1.18 \mathrm{e}-01$ \\
\hline 2 & $2.74 \mathrm{e}-02$ & $5.22 \mathrm{e}-02$ & $5.90 \mathrm{e}-02$ \\
\hline 3 & $1.36 \mathrm{e}-02$ & $2.59 \mathrm{e}-02$ & $2.93 \mathrm{e}-02$ \\
\hline 4 & $6.64 \mathrm{e}-03$ & $1.26 \mathrm{e}-02$ & $1.43 \mathrm{e}-02$ \\
\hline
\end{tabular}

Table 2: Comparison, in the case of mesh 3, for different refinements (column one), of the $H^{1}$ relative error in the domain composed by subdomains $\Omega^{1}, \Omega^{2}$ and $\Omega^{3}$

(column 2) to the $H^{1}$ relative error in the subdomain $\Omega^{4}$ (column 3). The fourth column is the $H^{1}$ relative error in the whole domain.

Let us now do the same comparison in the case of mesh 3. This mesh is coarser in the subdomain $\Omega^{4}$ where the solution steeply varies. In table 2 , we observe that as expected, the $H^{1}$ relative error in the domain composed by subdomains $\Omega^{1}, \Omega^{2}$ and $\Omega^{3}$ (second column of table 2) is smaller (almost half) than the $H^{1}$ relative error in the subdomain $\Omega^{4}$ (third column of table 2). That one is close to the $H^{1}$ relative error in the whole domain (fourth column of table 2), because mesh 3 is coarser in the subdomain $\Omega^{4}$ where the solution steeply varies.

4.4. Convergence : Choice of the Robin parameter. Let us now study the convergence speed to reach the discrete solution, for different values of the Robin parameter $\alpha$. We first consider a domain decomposition in two subdomains, and then in four subdomains.

4.4.1. 2 subdomain case. In this part, the unit square is decomposed in two subdomains with non-conforming meshes (with 81 and 153 nodes respectively) as shown in figure 4.7. On figure 4.8 we represent the relative $H^{1}$ error between the discrete Schwarz converged solution and the iterate solution, for different values of the Robin parameter $\alpha$. We observe that the optimal numerical value of the Robin parameter is close to $\alpha_{\text {mean }}$ and near $\alpha_{\min }$ and $\alpha_{\max }$. 


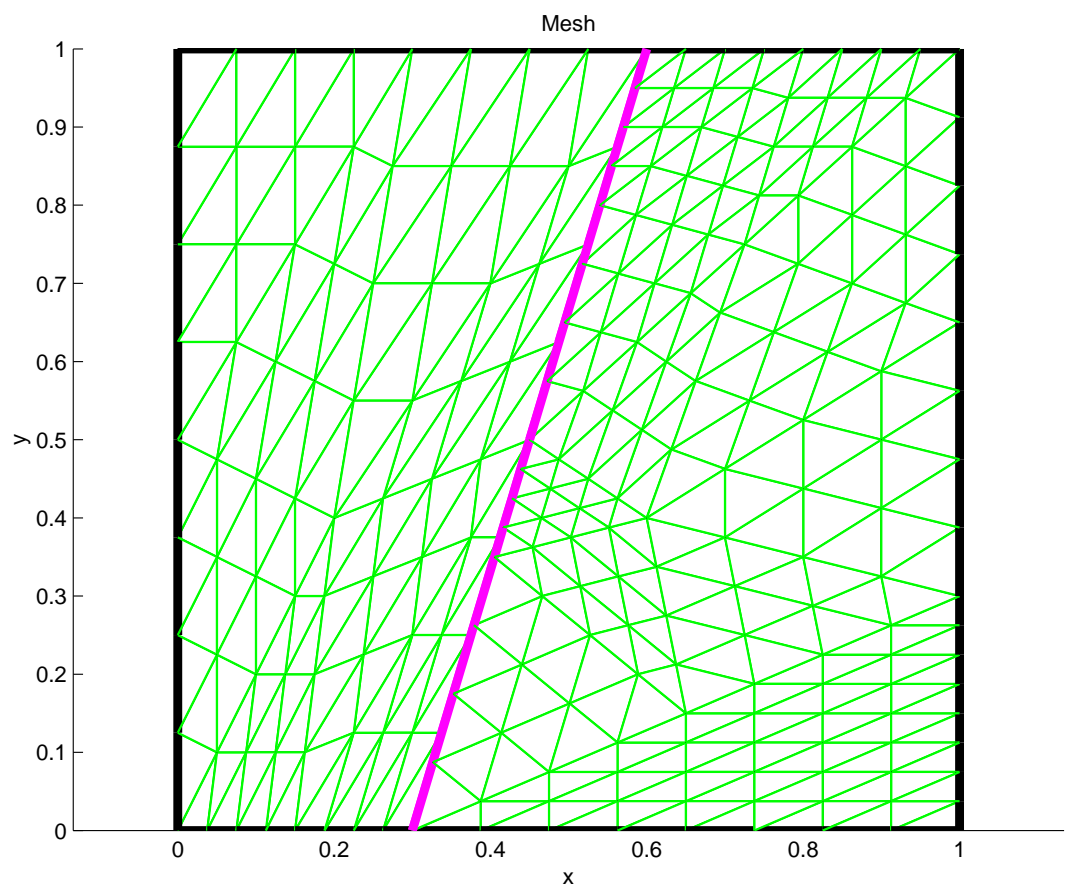

FIG. 4.7. Domain decomposition in 2 subdomains with non-conforming grids

As the relative $H^{1}$ error didn't show where the error is highest, we also look at the relative $L^{\infty}$ error between the discrete Schwarz converged solution and the solution at iteration $p$, for different values of the Robin parameter $\alpha$. We obtain similar results as for the relative $H^{1}$ error (see figure 4.9).

The Schwarz algorithm can be interpreted as a Jacobi algorithm applied to an interface problem (see [32]). In order to accelerate the convergence, we can replace the Jacobi algorithm by a Gmres ([33]) algorithm. Figures 4.10 and 4.11 show respectively the relative $H^{1}$ error and the relative $L^{\infty}$ error between the discrete Gmres converged solution and the iterate solution, for different values of the Robin parameter $\alpha$. In the case where $\alpha=\alpha_{\text {mean }}$, we observe that the convergence is accelerated by a factor 2 for Gmres, compared to Schwarz algorithm. Also, the gap between the error values for different $\alpha$ is decreasing when using Gmres algorithm, compared to Schwarz method. The Gmres algorithm is less sensitive to the choice of the Robin parameter. 


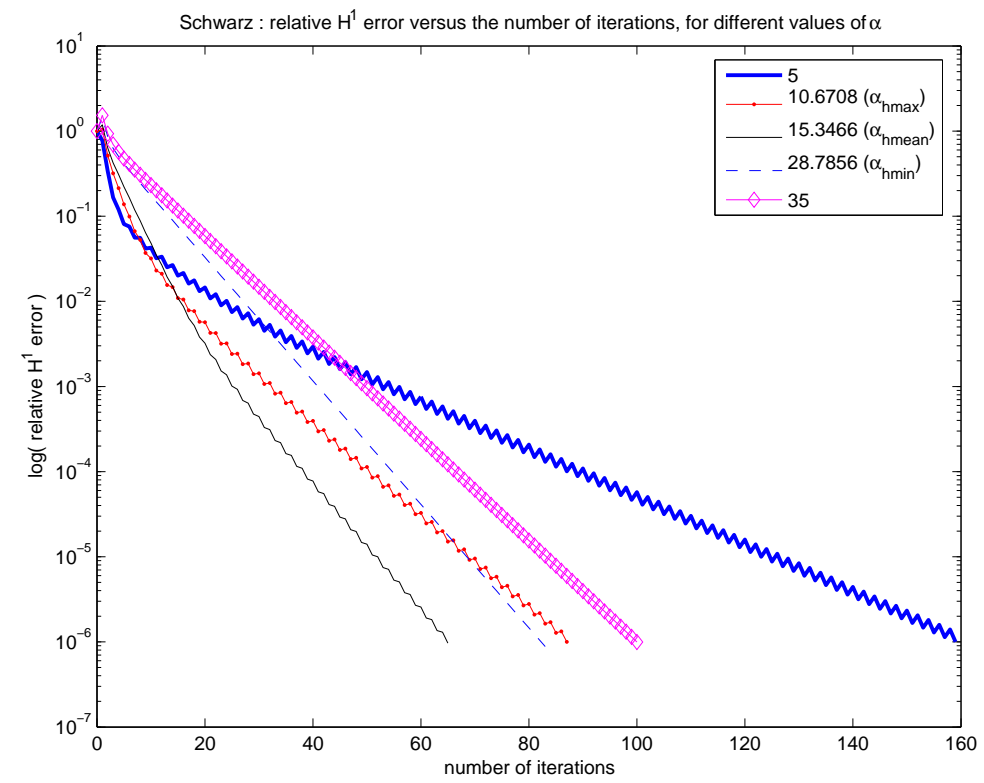

FIG. 4.8. relative $H^{1}$ error between the discrete Schwarz converged solution and the iterate solution, for different values of the Robin parameter $\alpha$

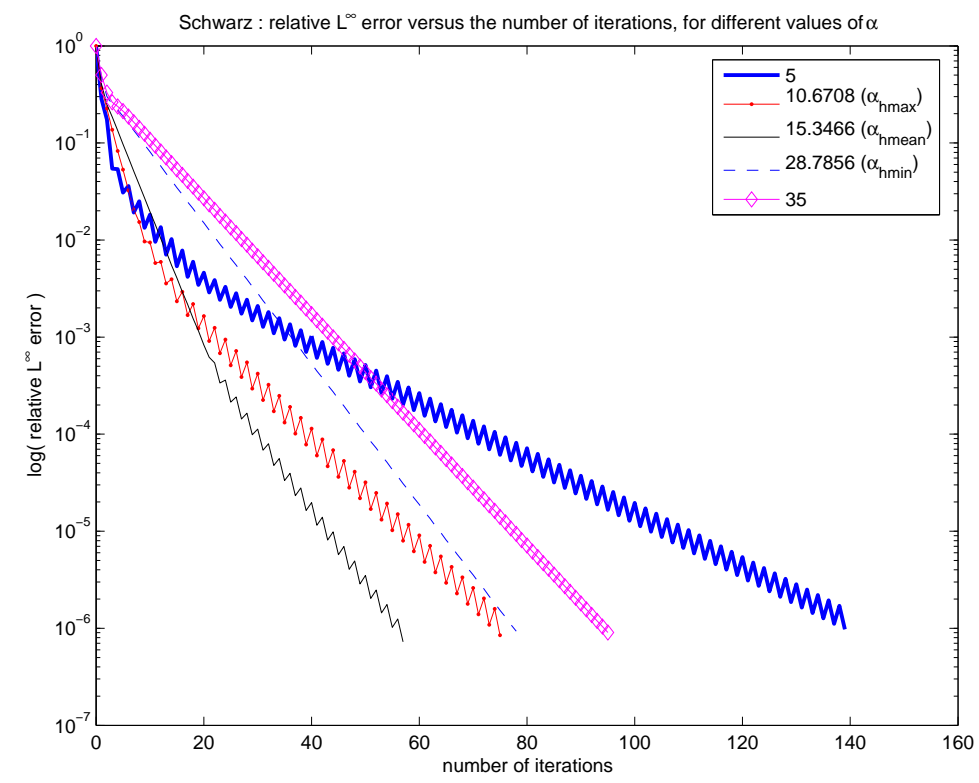

FIG. 4.9. Relative $L^{\infty}$ error between the discrete Schwarz converged solution and the iterate solution, for different values of the Robin parameter $\alpha$ 


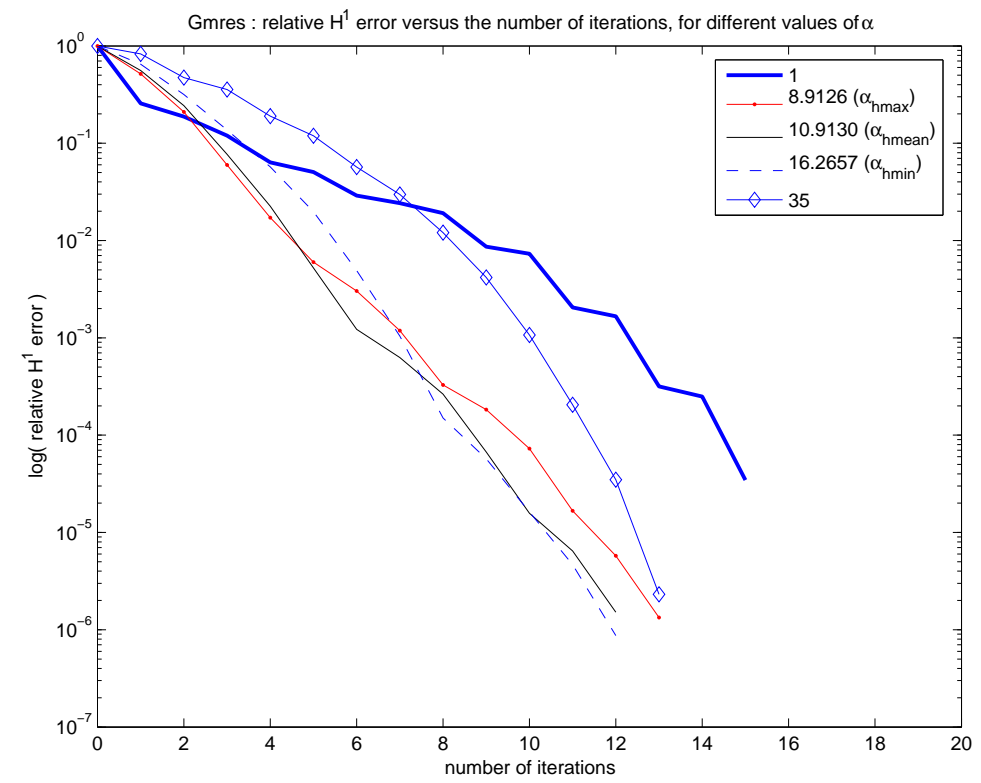

FIG. 4.10. relative $H^{1}$ error between the discrete Gmres converged solution and the iterate solution, for different values of the Robin parameter $\alpha$

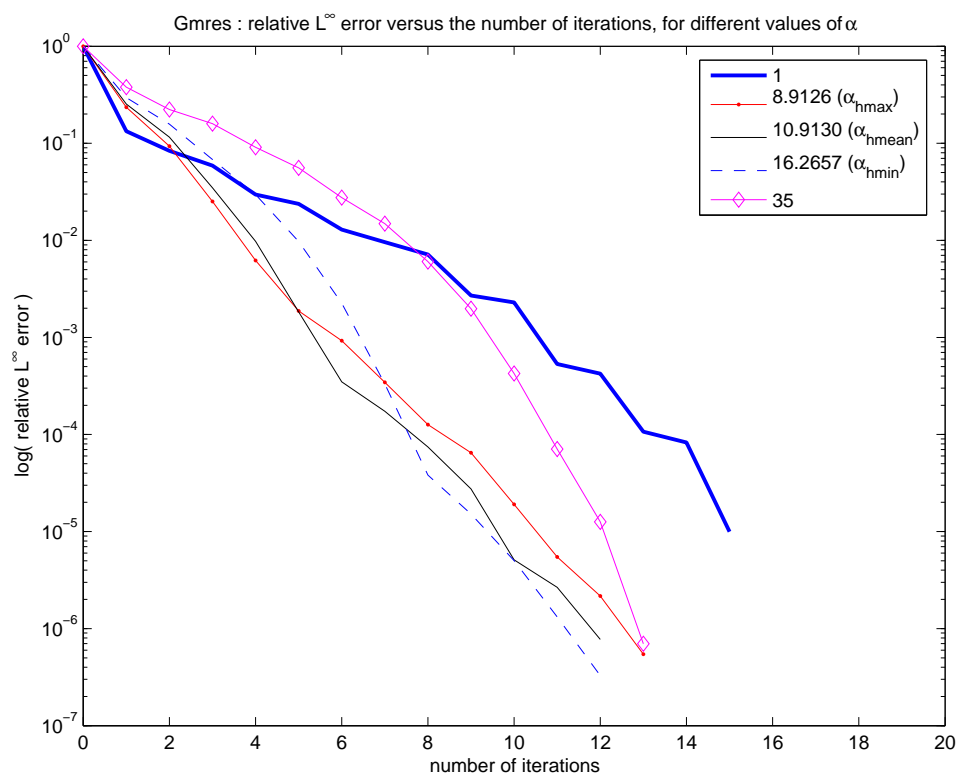

FIG. 4.11. Relative $L^{\infty}$ error between the discrete Gmres converged solution and the iterate solution, for different values of the Robin parameter $\alpha$

4.4.2. 4 subdomain case. In this part, the unit square is decomposed into four subdomains with non-conforming meshes (with 189, 81, 45 and 153 nodes respectively) as shown in figure 4.12. On figure 4.13 and 4.14 respectively, we represent the relative $H^{1}$ error and the relative $L^{\infty}$ error between the discrete Schwarz converged solution 
and the iterate solution, for different values of the Robin parameter $\alpha$. We observe that the optimal numerical value of the Robin parameter is close to $\alpha_{\text {mean }}$ and near $\alpha_{\min }$ and $\alpha_{\max }$, as in the two subdomain case.

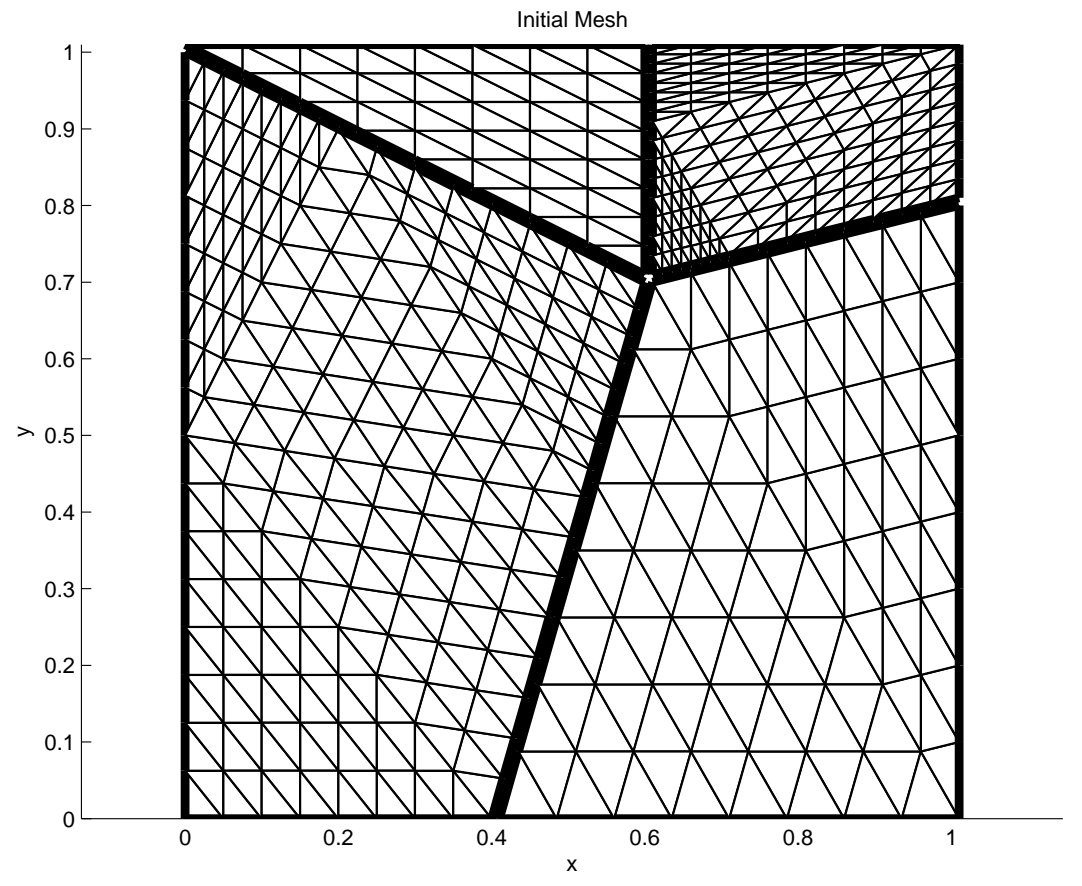

FIG. 4.12. Domain decomposition in 4 subdomains with non-conforming grids

4.4.3. Conclusions. The numerical results on the relative $H^{1}$ error between the continuous and discrete solutions correspond to the theoretical error estimates of theorem 4. On the other hand, we observe that, for a fixed number of mesh points, the relative $H^{1}$ error between the continuous and discrete solutions is smaller for a mesh refined in the region of the domain where the solution steeply varies, than for a mesh which is coarser in that region. In term of convergence speed to reach the discrete solution, the Robin parameter $\alpha$ must depend of the mesh size, and our simulations show that $\alpha=\alpha_{\text {mean }}$ is close to the optimal numerical value. 


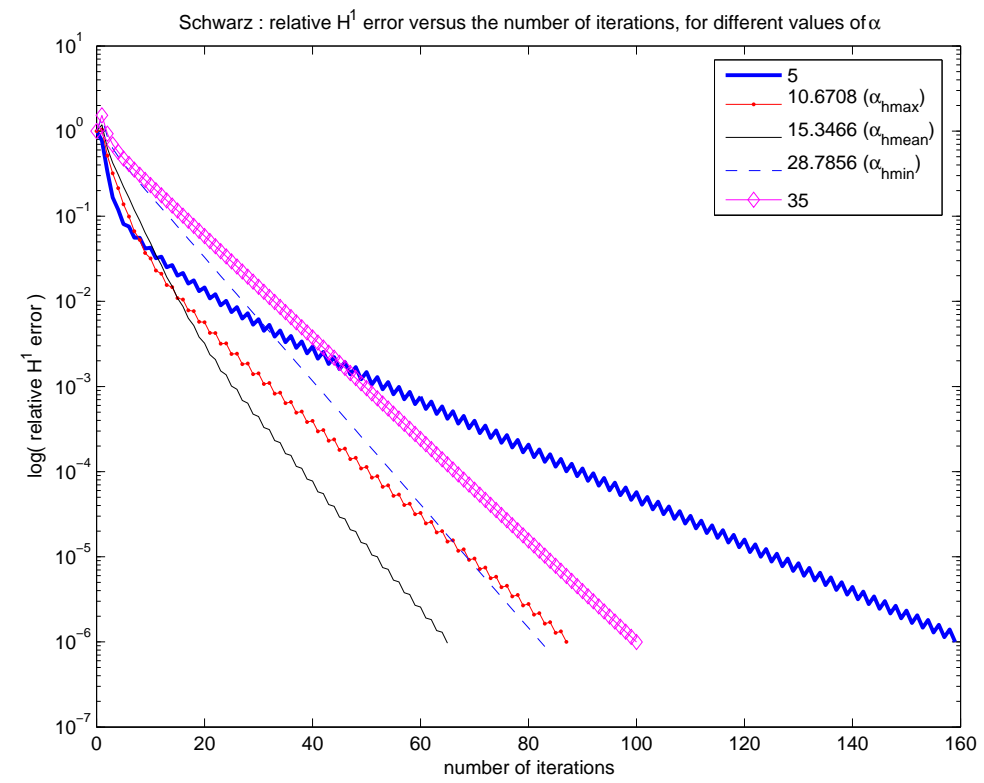

FIG. 4.13. relative $H^{1}$ error between the discrete Schwarz converged solution and the iterate solution, for different values of the Robin parameter $\alpha$

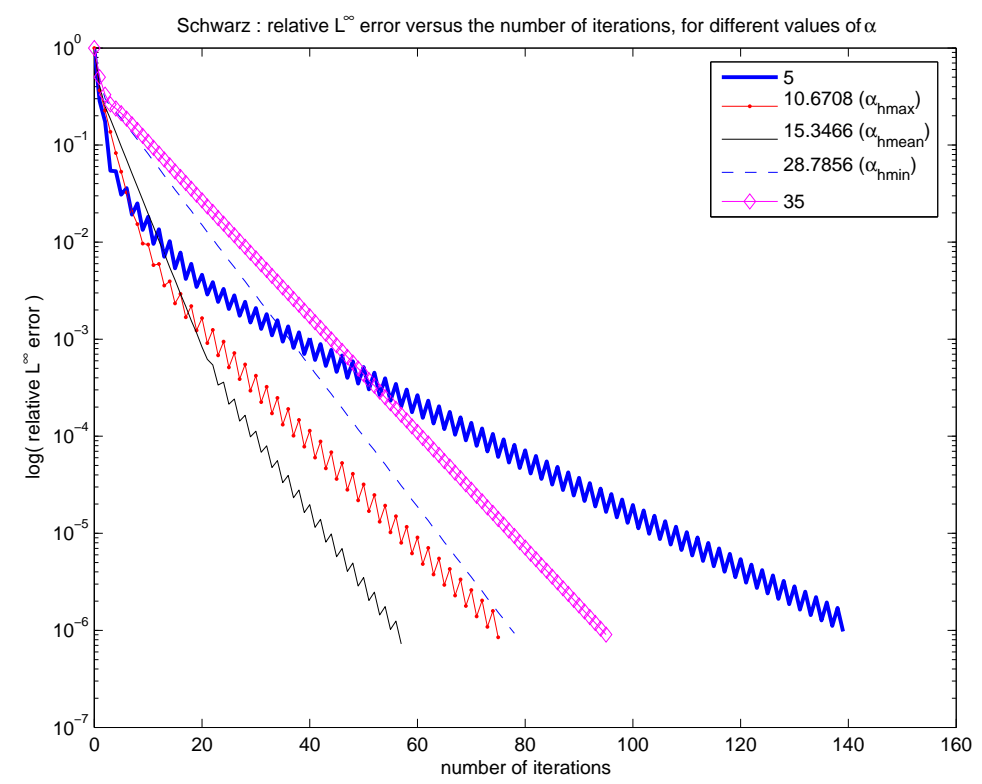

FIG. 4.14. Relative $L^{\infty}$ error between the discrete Schwarz converged solution and the iterate solution, for different values of the Robin parameter $\alpha$

Appendix A. Inf-sup condition.. The purpose of this annex is to show that the proof of 4 ] can be extended to the 3D situation. Indeed the main ingredients required for the extensions have been proven in [11. Let us first recall a standard 
stability result in higher norms of the $L^{2}$ projection operator $\bar{\pi}_{k, \ell}$ from $L^{2}\left(\Gamma^{k, \ell}\right)$ onto $\mathcal{Y}_{h}^{k, \ell} \cap H_{0}^{1}\left(\Gamma^{k, \ell}\right)$ orthogonal to $\tilde{W}_{h}^{k, \ell}$.

Lemma 7. Making the hypothesis that the triangulation $\mathcal{T}_{h}^{k}$ is uniformly regular, there exists a constant $c>0$ such that

$$
\forall v \in H_{00}^{\frac{1}{2}}\left(\Gamma^{k, \ell}\right),\left\|\bar{\pi}_{k, \ell} v\right\|_{H_{00}^{\frac{1}{2}}\left(\Gamma^{k, \ell}\right)} \leq c\|v\|_{H_{00}^{\frac{1}{2}}\left(\Gamma^{k, \ell}\right)} .
$$

Proof From (3.37) we deduce a uniform inf-sup condition between $\mathcal{Y}_{h}^{k, \ell} \cap H_{0}^{1}\left(\Gamma^{k, \ell}\right)$ and $\tilde{W}_{h}^{k, \ell}$ in $L^{2}\left(\Gamma^{k, \ell}\right)$. It results that the projection operator $\bar{\pi}$ is stable in $L^{2}\left(\Gamma^{k, \ell}\right)$ and thus there exists a constant $c_{1}>0$ such that

$$
\forall v \in H_{00}^{\frac{1}{2}}\left(\Gamma^{k, \ell}\right),\left\|v-\bar{\pi}_{k, \ell} v\right\|_{L^{2}\left(\Gamma^{k, \ell}\right)} \leq c_{1} h^{\frac{1}{2}}\|v\|_{H_{00}^{\frac{1}{2}}\left(\Gamma^{k, \ell}\right)} .
$$

Let $\tilde{\pi}_{k, \ell}$ denote the orthogonal projection operator from $H_{00}^{\frac{1}{2}}\left(\Gamma^{k, \ell}\right)$ onto $\mathcal{Y}_{h}^{k, \ell} \cap H_{0}^{1}\left(\Gamma^{k, \ell}\right)$ for $H_{00}^{\frac{1}{2}}\left(\Gamma^{k, \ell}\right)$ inner product. Then, for all $v$ in $H_{00}^{\frac{1}{2}}\left(\Gamma^{k, \ell}\right)$,

$$
\left\|\bar{\pi}_{k, \ell} v\right\|_{H_{00}^{\frac{1}{2}}\left(\Gamma^{k, \ell}\right)} \leq\left\|\tilde{\pi}_{k, \ell} v\right\|_{H_{00}^{\frac{1}{2}}\left(\Gamma^{k, \ell}\right)}+\left\|\bar{\pi}_{k, \ell} v-\tilde{\pi}_{k, \ell} v\right\|_{H_{00}^{\frac{1}{2}}\left(\Gamma^{k, \ell}\right)} .
$$

Then, with an inverse inequality, there exists a constant $c_{2}>0$ such that

$$
\left\|\bar{\pi}_{k, \ell} v\right\|_{H_{00}^{\frac{1}{2}}\left(\Gamma^{k, \ell}\right)} \leq\|v\|_{H_{00}^{\frac{1}{2}}\left(\Gamma^{k, \ell}\right)}+c_{2} h^{-\frac{1}{2}}\left\|\bar{\pi}_{k, \ell} v-\tilde{\pi}_{k, \ell} v\right\|_{L^{2}\left(\Gamma^{k, \ell}\right)} .
$$

Thus,

$$
\left\|\bar{\pi}_{k, \ell} v\right\|_{H_{00}^{\frac{1}{2}}\left(\Gamma^{k, \ell}\right)} \leq\|v\|_{H_{00}^{\frac{1}{2}}\left(\Gamma^{k, \ell}\right)}+c_{2} h^{-\frac{1}{2}} c^{\prime} h^{\frac{1}{2}}\|v\|_{H_{00}^{\frac{1}{2}}\left(\Gamma^{k, \ell}\right)},
$$

and then, with $c=1+c^{\prime} c_{2}$, we have

$$
\left\|\bar{\pi}_{k, \ell} v\right\|_{H_{00}^{\frac{1}{2}}\left(\Gamma^{k, \ell}\right)} \leq c\|v\|_{H_{00}^{\frac{1}{2}}\left(\Gamma^{k, \ell}\right)}, \forall v \in H_{00}^{\frac{1}{2}}\left(\Gamma^{k, \ell}\right),
$$

which ends the proof of lemma 7 .

Then from the definition of the $H_{*}^{-\frac{1}{2}}\left(\Gamma^{k, \ell}\right)$ norm, for any $p_{h, k, \ell}$ in $\tilde{W}_{h}^{k, \ell}$, there exists an element $w^{k, \ell}$ in $H_{00}^{\frac{1}{2}}\left(\Gamma^{k, \ell}\right)$ such that

$$
\int_{\Gamma^{k, \ell}} p_{h, k, \ell} w^{k, \ell}={ }_{\left(H_{00}^{1 / 2}\right)^{\prime}\left(\Gamma^{k, \ell}\right)}<p_{h, k, \ell}, w^{k, \ell}>_{H_{00}^{1 / 2}\left(\Gamma^{k, \ell}\right)}=\left\|p_{h, k, \ell}\right\|_{\left(H_{00}^{\frac{1}{2}}\left(\Gamma^{k, \ell}\right)\right)^{\prime}}\left\|w^{k, \ell}\right\|_{H_{00}^{\frac{1}{2}}\left(\Gamma^{k, \ell}\right)}
$$

and $w^{k, \ell}$ can be chosen such that

$$
\left\|w^{k, \ell}\right\|_{H_{00}^{\frac{1}{2}}\left(\Gamma^{k, \ell}\right)}=\left\|p_{h, k, \ell}\right\|_{\left(H_{00}^{\frac{1}{2}}\left(\Gamma^{k, \ell}\right)\right)^{\prime}} .
$$

We apply now the projection operator on $w^{k, \ell}$ from lemma 7 . We derive that $\bar{\pi}_{k, \ell}\left(w^{k, \ell}\right)=w_{h}^{k, \ell} \in \mathcal{Y}_{h}^{k, \ell} \cap H_{0}^{1}\left(\Gamma^{k, \ell}\right)$ and

$$
\left\|w_{h}^{k, \ell}\right\|_{H_{00}^{\frac{1}{2}}\left(\Gamma^{k, \ell}\right)} \leq c\left\|p_{h, k, \ell}\right\|_{\left(H_{00}^{\frac{1}{2}}\left(\Gamma^{k, \ell}\right)\right)^{\prime}},
$$


and

$$
\int_{\Gamma^{k, \ell}} p_{h, k, \ell} w_{h}^{k, \ell}=\int_{\Gamma^{k, \ell}} p_{h, k, \ell} w^{k, \ell}=\left\|p_{h, k, \ell}\right\|_{\left(H_{00}^{\frac{1}{2}}\left(\Gamma^{k, \ell}\right)\right)^{\prime}}^{2} .
$$

It remains to lift $w_{h}^{k, \ell}$ over $\Omega^{k}$, this is done by prolongating $w_{h}^{k, \ell}$ by zero over $\partial \Omega^{k} \backslash \Gamma^{k, \ell}$ and lifting this element of $H^{\frac{1}{2}}\left(\partial \Omega^{k}\right)$ over $\Omega^{k}$ as proposed in [7].

\section{Appendix B. Extension in 2D for high order approximations.}

Lemma 8. Let $1 \leq p \leq 13$ be an integer. There exists $c$ and $C>0$ such that for all $\eta \in \mathbf{P}^{p}([-1,1])$ s.t. $\eta(-1)=0$ there exists $\psi \in \mathbf{P}^{p-1}([-1,1])$ s.t.

$$
\eta(1)=\psi(1)
$$

and

$$
J(\psi ; \eta):=\int_{-1}^{1}\left(\eta \psi-\frac{1}{4}(\eta-\psi)^{2}\right) \geq c \int_{-1}^{1} \eta^{2}
$$

and

$$
\int_{-1}^{1} \psi^{2} \leq C \int_{-1}^{1} \eta^{2}
$$

This lemma has been proven in the case $p=1$ at section 3.2. For $p \geq 2$, we prove this lemma by studying for a given $\eta \in \mathbf{P}^{p}([-1,1]), \eta \neq 0$ the maximization problem

Find $\psi \in \mathbf{P}^{p-1}([-1,1])$ such that

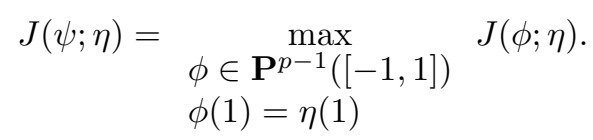

The function $J$ is strictly concave and there exists a function satisfying the constraint. This problem admits a solution. The functional $J(\phi, \eta)$ being quadratic in $(\phi, \eta)$ and the constraint being affine, the optimality condition shows that the problem reduces to a linear problem whose right hand side depends linearly of $\eta$. The affine constraint being of rank one, the problem (B.1) admits a unique solution which depends linearly of $\eta$. Therefore, it makes sense to introduce the operator:

$$
\begin{aligned}
S: \mathbf{P}_{0}^{p}([-1,1]) & \longrightarrow \mathbf{P}^{p-1}([-1,1]) \\
\eta & \mapsto \psi \text { solution to (B.1) }
\end{aligned}
$$

where $\mathbf{P}_{0}^{p}([-1,1])$ is the set of functions of $\mathbf{P}^{p}([-1,1])$ that vanish at -1 . In Lemma 8 we take $\psi=S(\eta)$. The operator $S$ is linear from a finite dimensional space to another so that it is continuous for any norm on these spaces. Therefore there exists $C>0$ such that $\int_{-1}^{1} \psi^{2} \leq C \int_{-1}^{1} \eta^{2}$. Moreover, the function

$$
\begin{aligned}
H: \mathbf{P}_{0}^{p}([-1,1]) \backslash\{0\} & \longrightarrow \mathbb{R} \\
\eta & \mapsto \frac{J(S(\eta), \eta)}{\int_{-1}^{1} \eta^{2}}
\end{aligned}
$$


is continuous and such that $H(\eta)=H(\alpha \eta)$ for any $\alpha \neq 0$. Therefore, it reaches its minimum and proving Lemma 8 amounts to prove

Lemma 9. Let $p \leq 13$ and $\eta \in \mathbf{P}^{p}([-1,1])$ s.t. $\eta(-1)=0$ and $\eta$ is not the null function.

Then,

$$
J(S(\eta) ; \eta)>0
$$

Proof. We make use of the Legendre polynomials

$$
L_{0}(x)=1, L_{1}(x)=x,(m+1) L_{m+1}(x)=(2 m+1) x L_{m}(x)-m L_{m-1}(x), m \geq 1
$$

Let us recall that for any $m \geq 0$,

$$
\begin{aligned}
& L_{m}(1)=1, L_{m}(-1)=(-1)^{m} \\
& \int_{-1}^{1} L_{m}(x) L_{m^{\prime}}(x) d x=\delta_{m m^{\prime}} \frac{2}{2 m+1}
\end{aligned}
$$

The polynomial $\eta$ is decomposed on the Legendre polynomials

$$
\eta=\sum_{m=1}^{p} \eta_{m}\left(L_{m}+L_{m-1}\right)
$$

and $\psi=S(\eta)$ is sought in the form

$$
\psi=\sum_{m=0}^{p-1} \psi_{m} L_{m}
$$

so that it maximizes the quantity $J(\psi ; \eta)$ under the constraint $\eta(1)=\psi(1)$. This corresponds to the min-max problem

$$
\max _{\psi \in \mathbf{P}^{p-1}([-1,1])} \min _{\mu \in \mathbb{R}} \mathcal{L}(\psi, \mu)
$$

where

$$
\mathcal{L}(\psi, \mu)=J(\psi ; \eta)-\mu(\psi(1)-\eta(1)) .
$$

We have to prove that the optimal value is positive. The optimality relations w.r.t $\psi$ give

$$
\frac{3}{2}\left(\eta_{m}+\eta_{m+1}\right)-\frac{1}{2} \psi_{m}=\mu \frac{2 m+1}{2}, 1 \leq m \leq p-1
$$

and

$$
\frac{3}{2} \eta_{1}-\frac{1}{2} \psi_{0}=\frac{\mu}{2}
$$

Therefore, we get

$$
\psi=3 \eta-3 \eta_{p} L_{p}-\mu R_{p-1}
$$


where $R_{p-1}=\sum_{m=0}^{p-1}(2 m+1) L_{m}$ and $\left\|R_{p-1}\right\|_{L^{2}(]-1,1[)}^{2}=2 p^{2}$. Hence, the dual problem writes

$$
\min _{\mu \in \mathbb{R}} G(\mu ; \eta)
$$

where

$$
G(\mu ; \eta):=J\left(3 \eta-3 \eta_{p} L_{p}-\mu R_{p-1} ; \eta\right)-\mu(\psi(1)-\eta(1))
$$

and $\psi$ satisfies (B.2). After some calculations, we get

$$
G(\mu ; \eta)=\frac{p^{2}}{2} \mu^{2}-\mu\left(2 \eta(1)-3 \eta_{p}\right)+\left(2\|\eta\|_{L^{2}(]-1,1[)}^{2}-\frac{9}{2} \frac{\eta_{p}^{2}}{2 p+1}\right) .
$$

The leading coefficient of $G(\mu ; \eta)$ is positive so that proving $\min _{\mu} G(\mu ; \eta)$ is positive (and hence Lemma 9) is equivalent to prove

Lemma 10. For $p \leq 13$, the discriminant of (B.3):

$$
\Delta(\eta):=\left(2 \eta(1)-3 \eta_{p}\right)^{2}+p^{2}\left(-4\|\eta\|_{L^{2}(]-1,1[)}^{2}+9 \frac{\eta_{p}^{2}}{2 p+1}\right)
$$

is negative if $\eta \in \mathbf{P}^{p}([-1,1]), \eta(-1)=0$ and $\eta$ is not the null function. Proof. We first treat separately the case $p=2$. In this case, a direct computation shows that

$$
\Delta(\eta)=-\frac{80}{3} \eta_{1}^{2}-\frac{40}{3} \eta_{2} \eta_{1}-\frac{133}{15} \eta_{2}^{2}
$$

The discriminant of the corresponding bilinear form is $-8632 / 9$. It is negative and the lemma is proved in this case.

We consider now the case $p \geq 3$. Let us introduce the vector space $Q^{p}=\{\eta \in$ $\mathbf{P}^{p}([-1,1])$ s.t. $\left.\eta(-1)=0\right\}$. The function $\Delta(\eta)$ is quadratic so that it suffices to study the extrema of $\Delta(\eta) /\|\eta\|_{L^{2}(]-1,1[)}^{2}$ over $Q^{p}$ or equivalently to prove that the associated symmetric quadratic form in negative, i.e. its eigenvalues are negative. They correspond to the Lagrange multiplier solutions $\mu_{1}$ of the following min-max problem

$$
\min _{\eta \in Q^{p}} \max _{\mu_{1} \in \mathbb{R}} \mathcal{L}_{e}\left(\eta, \mu_{1}\right)
$$

where

$$
\mathcal{L}_{e}\left(\eta, \mu_{1}\right):=\Delta(\eta)-\mu_{1}\left(\|\eta\|_{L^{2}(]-1,1[)}^{2}-1\right) .
$$

We have to prove that $\mu_{1}<0$. We have

$$
\begin{aligned}
0= & <\frac{\partial \mathcal{L}_{e}}{\partial \eta}, \delta \eta> \\
& =2\left(2 \eta(1)-3 \eta_{p}\right)\left(2 \delta \eta(1)-3 \delta \eta_{p}\right)+p^{2}\left(-8<\eta, \delta \eta>+18 \frac{\eta_{p} \delta \eta_{p}}{2 p+1}\right)-2 \mu_{1}<\eta, \delta \eta>
\end{aligned}
$$

where $<,>$ denotes the $L^{2}$ scalar product on $L^{2}(]-1,1[)$ and $\delta \eta \in Q^{p}$.

Let us consider the vector space $\left(1-x^{2}\right) \mathbf{P}^{p-3} \subset Q^{p}$. Any function $\gamma$ in $\left(1-x^{2}\right) \mathbf{P}^{p-3}$ 
satisfies $\gamma(-1)=\gamma(1)=0$ and $\gamma_{p}=0$. The optimality relation w.r.t. to $\left(1-x^{2}\right) \mathbf{P}^{p-3}$ gives

$$
\left(-8 p^{2}-2 \mu_{1}\right)<\eta, \delta \eta>=0, \quad \forall \delta \eta \in\left(1-x^{2}\right) \mathbf{P}^{p-3} .
$$

We have either $\mu_{1}=-4 p^{2}<0$ or $\eta$ solution to (B.5) belongs to the space $\{(1-$ $\left.\left.x^{2}\right) \mathbf{P}^{p-3}\right\}^{\perp} \cap \mathbf{P}^{p}$. The first case corresponds to a negative value for $\mu_{1}$ which is in agreement with the lemma to be proved. Let us study the latter case. We shall make use of

LEMMA 11

$$
\begin{array}{r}
\int_{-1}^{1} L_{m}^{\prime} L_{m^{\prime}}^{\prime}\left(1-x^{2}\right) d x=0, m \neq m^{\prime}, \\
\int_{-1}^{1} L_{m}^{\prime 2}=m(m+1), \\
\int_{-1}^{1} L_{m}^{\prime} L_{m+1}^{\prime}=0, \\
\int_{-1}^{1} L_{m-1}^{\prime} L_{m+1}^{\prime}=m(m-1), \\
L_{m}^{\prime}(-1)=(-1)^{m+1} \frac{m(m+1)}{2} .
\end{array}
$$

see [1]. From Lemma 11, it can be proved that

LEMMa 12.

$$
\left\{\left(1-x^{2}\right) \mathbf{P}^{p-3}\right\}^{\perp} \cap \mathbf{P}^{p}=\operatorname{Span}\left\{L_{p}, L_{p}^{\prime}, L_{p-1}^{\prime}\right\} .
$$

Proof. From (B.6), it can be checked easily that

$$
\left\{\left(1-x^{2}\right) \mathbf{P}^{p-3}\right\}^{\perp} \cap \mathbf{P}^{p}=\operatorname{Span}\left\{L_{p+1}^{\prime}, L_{p}^{\prime}, L_{p-1}^{\prime}\right\} .
$$

Moreover, we have

$$
\left.L_{p+1}^{\prime}(x)=(2 p+1) L_{p}(x)+L_{p-1}^{\prime}(x)\right)
$$

and thus lemma 12 .

Therefore, there exists $\lambda_{1}, \lambda_{2}, \lambda_{3} \in \mathbb{R}$ s.t. $\eta=\lambda_{1} L_{p}+\lambda_{2} L_{p}^{\prime}+\lambda_{3} L_{p-1}^{\prime}$. Since $\eta$ is defined up to a constant and we only have to consider the two cases $\lambda_{1}=1$ or $\lambda_{1}=0$. Case $1 \lambda_{1}=1$

From $\eta(-1)=0$, we get

$$
1-\lambda_{2} \frac{p(p+1)}{2}+\lambda_{3} \frac{p(p-1)}{2}=0
$$

so that

$$
\begin{gathered}
\lambda_{2}=\frac{2}{p(p+1)}+\lambda_{3} \frac{p-1}{p+1} . \\
\Delta(\eta)=-4 \frac{(p-1) p^{2}\left(p^{2}+1\right)}{p+1} \lambda_{2}^{2}-\frac{\left(24 p^{4}-20 p^{3}-8 p^{2}+4 p\right)}{(p+1)(2 p+1)} \lambda_{2} \\
-\frac{29 p^{2}+13 p-1-p^{3}}{(p+1)(2 p+1)}
\end{gathered}
$$


Since $p$ is supposed larger than 1 , the leading coefficient of $\Delta(\eta)$ is negative. If the discriminant of $\Delta(\eta)$ is negative, the polynomial is negative for any $\lambda_{2}$. This discriminant has the value

$$
16 \frac{\left(p^{2}-13 p-8\right)(p-1) p^{3}}{2 p+1}
$$

and is negative for $2 \leq p \leq 13$.

Case $2 \lambda_{1}=0$

From $\eta(-1)=0$, we get

$$
-\lambda_{2} \frac{p(p+1)}{2}+\lambda_{3} \frac{p(p-1)}{2}=0
$$

so that

$$
\lambda_{2}=\lambda_{3} \frac{p-1}{p+1} .
$$

Since $\eta$ is an eigenvalue, it is not zero and the above relation shows that we can take $\lambda_{3}=1$. Then, we have $\lambda_{2}=\frac{p-1}{p+1}$ so that

$$
\Delta(\eta)=\frac{-4(p-1) p^{2}\left(p^{2}+1\right)}{(p+1)}<0 .
$$

Acknowledgments. The authors would like to thank Martin J. Gander for his help in the implementation of the method, especially for computing projections between arbitrary grids in two dimensions.

\section{REFERENCES}

[1] M. Abramowitz And I. A. Stegun, Handbook of mathematical functions with formulas, graphs, and mathematical tables, Dover Publications Inc., New York (1992), Reprint of the 1972 edition

[2] Y. Achdou, C. Japhet, Y. Maday and F. Nataf, A new cement to glue non-conforming grids with Robin interface conditions: the finite volume case, Numer. Math., 92 (2002), No. 4, pp. 593-620.

[3] Y. Achdou, Y. Maday and O. Widlund, Iterative Substructuring Preconditioners for Mortar Element Methods in Two Dimensions, SIAM J. Numer. Anal., 2 (1999), pp. 551-580.

[4] F. Ben Belgacem, The Mortar finite Element Method with Lagrange Multipliers, Numer. Math., 84(2) (1999), pp. 173-197.

[5] F. Ben Belgacem and Y. Maday, Coupling spectral and finite elements for second order elliptic three-dimensional equations, SIAM J. Numer. Anal., 36 (1999), No. 4, pp. 12341263.

[6] J.D. Benamou and B. Desprès, A Domain Decomposition Method for the Helmholtz equation and related Optimal Control Problems, J. Comp. Phys., 136 (1997) pp. 68-82.

[7] C. Bernardi and V. Girault, A local regularization operator for triangular and quadrilateral finite elements, SIAM J. Numer. Anal., 35 (1998), No. 5, pp. 1893-1916. Kiel (1986)

[8] C. Bernardi, Y. Maday and A. Patera, A new nonconforming approach to domain decomposition: the mortar element method, Nonlinear Partial Differential Equations and their Applications, eds H. Brezis and J.L. Lions, Pitman (1989).

[9] A. de la Bourdonnaye, C. Fahrat, A. Macedo, F. Magoulès and F.X. Roux, A Nonoverlapping Domain Decomposition Method for the Exterior Helmholtz Problem, DD10 Proceedings, (1997).

[10] D. Braess, Finite Elements: Theory, Fast Solvers and Applications in Solid Mechanics, Cambridge University Press, 1997. 
[11] D. Braess and W. Dahmen, Stability estimates of the mortar finite element method for 3dimensional problems, East-West J. Numer. Math., 6 (1998), No. 4, pp. 249-263.

[12] F. Brezzi And M. Fortin, Mixed and Hybrid Finite Element Methods, Springer-Verlag, New York - Berlin - Heidelberg (1991).

[13] M. Casarin, F. Elliot and O. Windlund, An Overlapping Schwarz algorithm for solving the Helmholtz equation, DD10 Proceedings (1997)

[14] B. Desprès, Domain decomposition method and the Helmholtz problem, Mathematical and Numerical aspects of wave propagation phenomena, SIAM, (1991) pp. 44-52.

[15] B. Desprès, Domain decomposition method and the Helmholtz problem. II, Kleinman Ralph (eds) et al., Mathematical and numerical aspects of wave propagation. Proceedings of the 2nd international conference held in Newark, DE, USA, June 7-10, 1993. Philadelphia, PA: SIAM, (1993), pp. 197-206.

[16] B. Despres, P. Joly and J. E. Roberts, International Symposium on Iterative methods in linear algebra, Brussels, Belgium, (1991), pp. 475-484.

[17] B. Engquist and A. Majda, Absorbing Boundary Conditions for the Numerical Simulation of Waves, Math. Comp., 31 (139), (1977) pp. 629-651.

[18] M. J. Gander, C. Japhet, Y. Maday, F. Nataf, it A new cement to glue non-conforming grids with Robin interface conditions: the finite element case, Domain Decomposition Methods in Science and Engineering Series : Lecture Notes in Computational Science and Engineering, Vol. 40, Kornhuber, R.; Hoppe, R.; Periaux, J.; Pironneau, O.; Widlund, O.; $\mathrm{Xu}$, J. (Eds.), (2004).

[19] M. J. Gander, L. Halpern and F. NAtaf, Optimal Schwarz Waveform Relaxation for the One Dimensional Wave Equation, SIAM J. Num. An., 41 (2003), No. 5, pp. 1643-1681.

[20] F. Gastaldi , L. Gastaldi and A. Quarteroni, Adaptative Domain Decomposition Methods for Advection dominated Equations, East-West J. Numer. Math., 4 (1996), pp. 165-206.

[21] S. Ghanemi, Méthode de décomposition de domaines avec conditions de transmissions non locales pour des problèmes de propagation d'ondes, Thèse de l'université de Paris IX Dauphine (1996)

[22] S. Ghanemi \& P. Joly \& F. Collino, Domain decomposition method for harmonic wave equations, Third international conference on mathematical and numerical aspect of wave propagation, (1995), pp. 663-672.

[23] T. Hagstrom, R. P. Tewarson and A. Jazcilevich, Numerical Experiments on a Domain Decomposition Algorithm for Nonlinear Elliptic Boundary Value Problems, Appl. Math. Lett., 1 (1988), No. 3, pp. 299-302.

[24] C. Japhet, Optimized Krylov-Ventcell Method. Application to Convection-Diffusion Problems, Proceedings of the $9^{\text {th }}$ International Conference on Domain Decomposition Methods, 3-8 june 1996, Bergen (Norway), Domain Decomposition Methods in Sciences and Engineering, edited by P. Bjorstad, M. Espedal and D. Keyes (1998), p. 382-389.

[25] C. Japhet, F. Nataf and F. Rogier, The Optimized Order 2 Method. Application to convection-diffusion problems, Future Generation Computer Systems, 18(1) (2001), pp. 17-30, Elsevier Science.

[26] S. C. Lee, M. N. Vouvakis and J. F. Lee, A non-overlapping domain decomposition method with non-matching grids for modeling large finite antenna arrays, J. Comput. Phys., 203 (1) (2005), pp. 1-21.

[27] L.C. McInnes, R.F. Susan-Resiga, D. E. Keyes and H. M. Atassi, Additive Schwarz methods with nonreflecting boundary conditions for the parallel computation of Helmholtz problems, in Xiao-Chuan Cai, Charbel Farhat and Jan Mandel, editors, Tenth International Symposium on Domain Decomposition Methods for Partial Differential Equations, AMS, (1997).

[28] C. Lacour, Analyse et Résolution Numérique de Méthodes de Sous-Domaines Non Conformes pour des Problèmes de Plaques, PhD thesis, Université Pierre et Marie Curie (1997).

[29] B. Lichtenberg, B. Webb, D. Meade and A. F. Peterson, Comparison of two-dimensional conformal local radiation boundary conditions, Electromagnetics 16, (1996), pp. 359-384.

[30] P.L. Lions, On the Schwarz Alternating Method III: A Variant for Nonoverlapping Subdomains, Third International Symposium on Domain Decomposition Methods for Partial Differential Equations, SIAM (1989), pp. 202-223.

[31] F. Nataf And F. Rogier, Factorization of the Convection-Diffusion Operator and the Schwarz Algorithm, $\mathrm{M}^{3} \mathrm{AS}, 5$ (1995), No. 1, pp. 67-93.

[32] F. Nataf, F. Rogier and E. De Sturler, Domain Decomposition Methods for Fluid Dynamics, Navier-Stokes Equations and Related Nonlinear Analysis, Edited by A. Sequeira, Plenum Press Corporation, (1995), pp. 367-376.

[33] SaAd, Youcef and Schultz, Martin H., GMRES: a generalized minimal residual algorithm for solving nonsymmetric linear systems, SIAM J. Sci. Statist. Comput., Society for In- 
dustrial and Applied Mathematics. Journal on Scientific and Statistical Computing, vol. 7, 1986, no 3, pp 856-869.

[34] L. Saas, I. Faille, F. Nataf and F. Willien, Finite Volume Methods for Domain Decomposition on Nonmatching Grids with Arbitrary Interface Conditions, SIAM J. Num. Ana., 43 (2005), No. 2, pp. 860-890.

[35] A. Toselli And O. Widlund, Domain decomposition methods-algorithms and theory, Springer Series in Computational Mathematics, 34 (2005), Springer-Verlag.

[36] O.B. WidLund, An extension theorem for finite element spaces with three applications, Numerical Techniques in Continuum Mechanics, Proc. Second GAMM Seminar, Kiel (1986). 

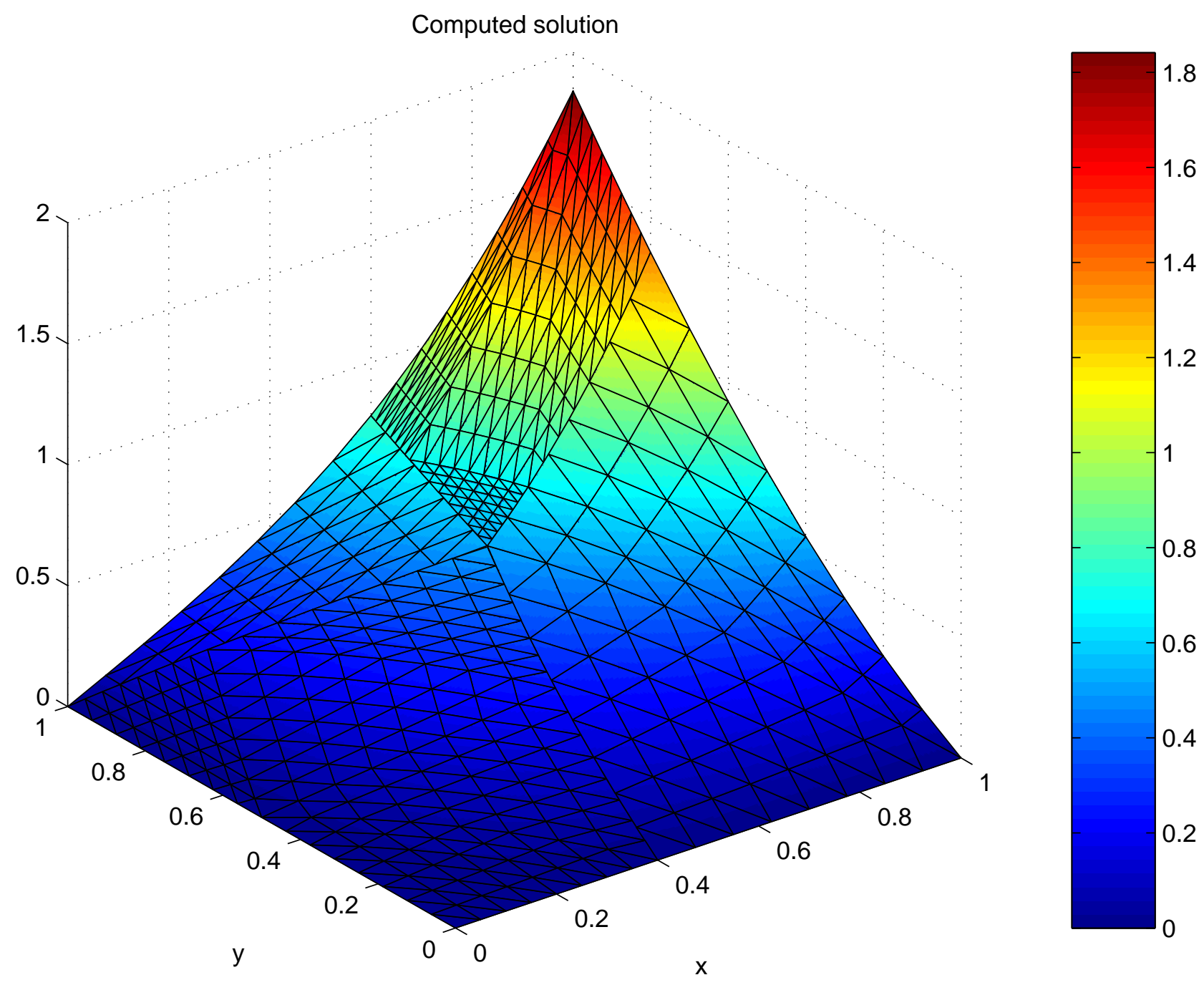


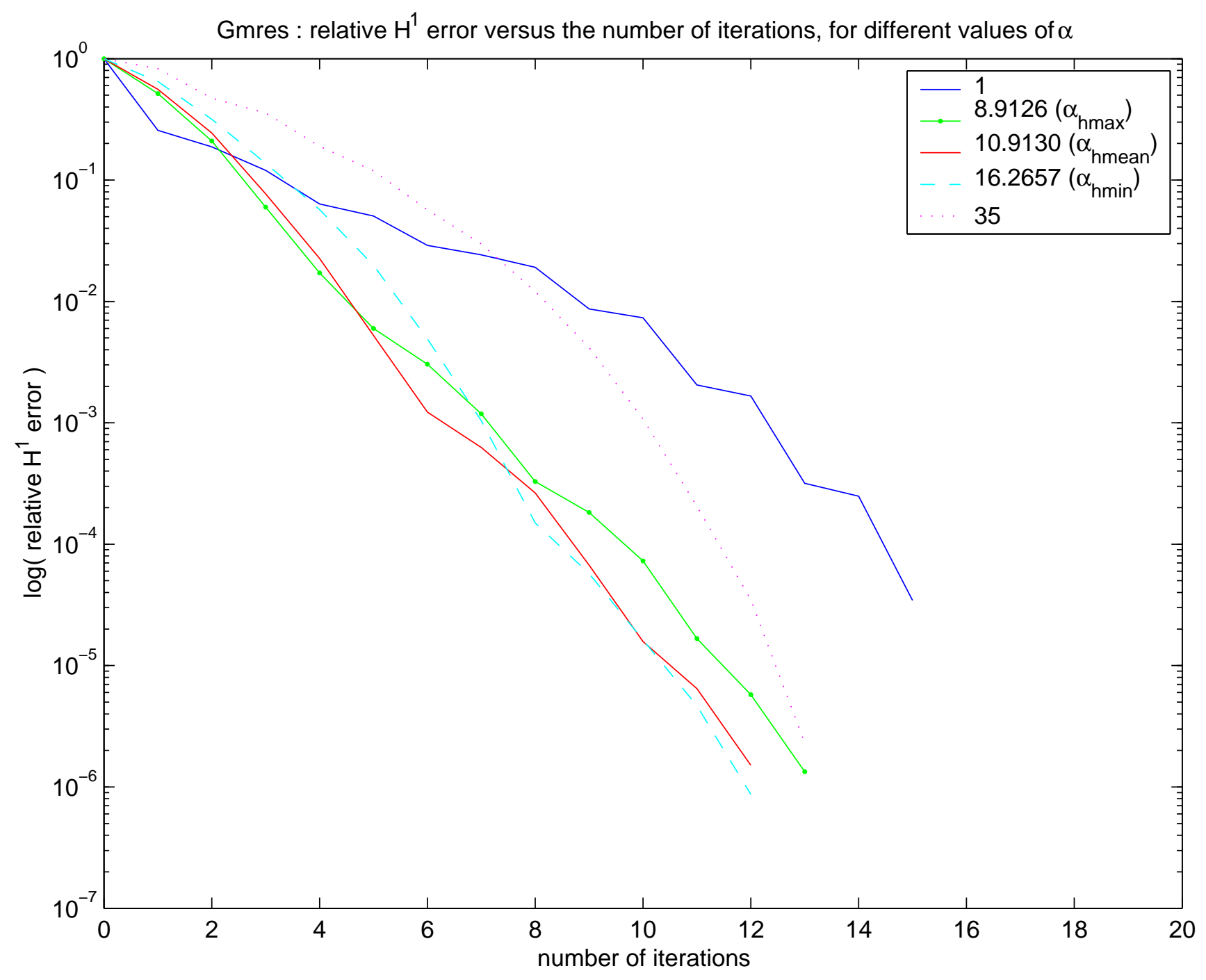




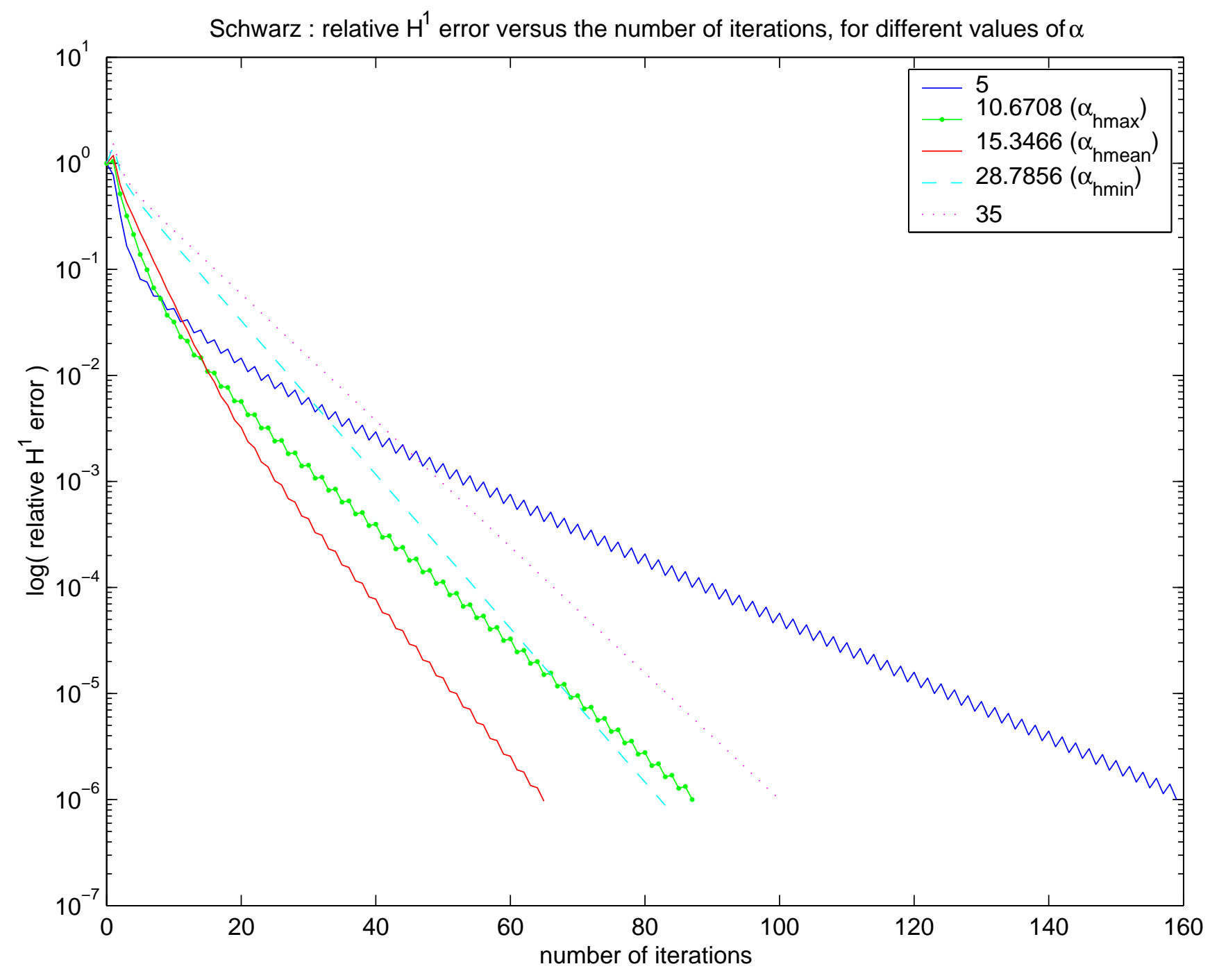




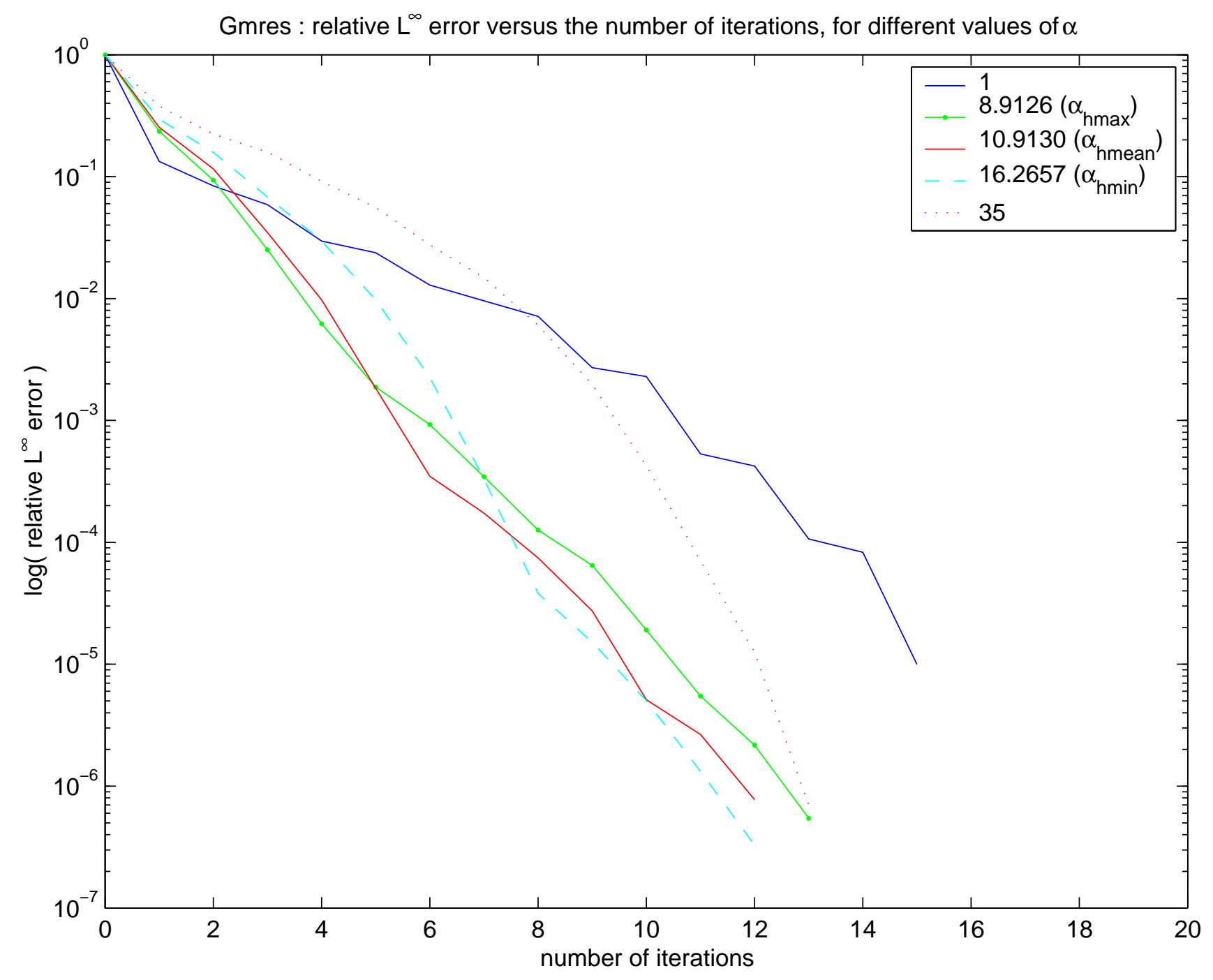




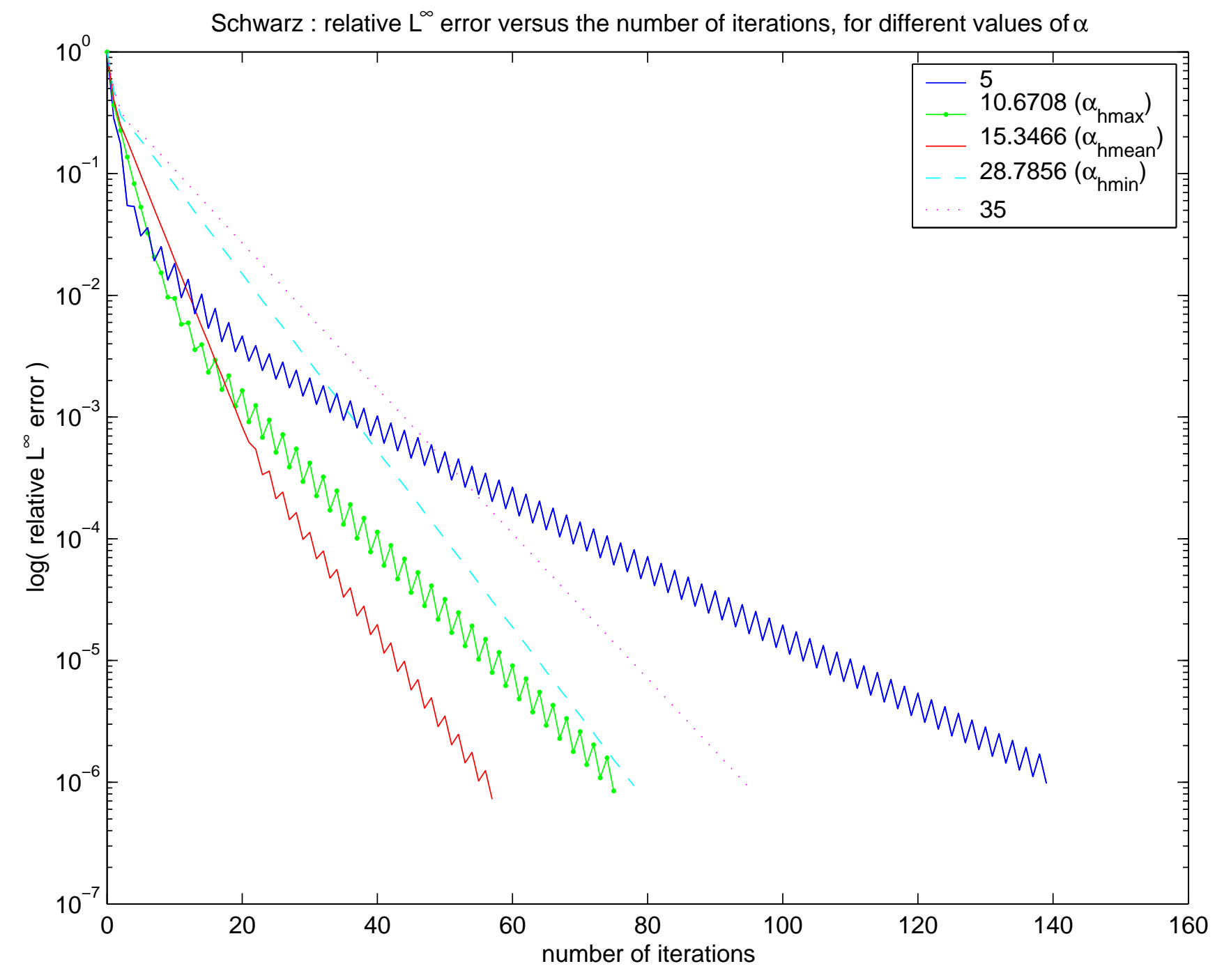

